Portland State University

PDXScholar

Fall 12-2-2015

\title{
Advances in Autonomous-Underwater-Vehicle Based Passive Bottom-Loss Estimation by Processing of Marine Ambient Noise
}

Lanfranco Muzi

Portland State University

Follow this and additional works at: https://pdxscholar.library.pdx.edu/open_access_etds

Part of the Electrical and Computer Engineering Commons, Ocean Engineering Commons, and the Remote Sensing Commons

Let us know how access to this document benefits you.

\section{Recommended Citation}

Muzi, Lanfranco, "Advances in Autonomous-Underwater-Vehicle Based Passive Bottom-Loss Estimation by Processing of Marine Ambient Noise" (2015). Dissertations and Theses. Paper 2612.

https://doi.org/10.15760/etd.2608

This Dissertation is brought to you for free and open access. It has been accepted for inclusion in Dissertations and Theses by an authorized administrator of PDXScholar. Please contact us if we can make this document more accessible: pdxscholar@pdx.edu. 
Advances in Autonomous-Underwater-Vehicle Based Passive BottomLoss Estimation by Processing of Marine Ambient Noise

\author{
by \\ Lanfranco Muzi
}

A dissertation submitted in partial fulfillment of the requirements for the degree of

\author{
Doctor of Philosophy \\ in \\ Electrical and Computer Engineering
}
Dissertation Committee:
Martin Siderius, Chair
Lisa M. Zurk
Richard Campbell
Randy Zelick

Portland State University

2015 
(C) 2015 Lanfranco Muzi 


\begin{abstract}
Accurate modeling of acoustic propagation in the ocean waveguide is important to SONAR-performance prediction, and requires, particularly in shallow water environments, characterizing the bottom reflection loss with a precision that databank-based modeling cannot achieve. Recent advances in the technology of autonomous underwater vehicles (AUV) make it possible to envision a survey system for seabed characterization composed of a short array mounted on a small AUV. The bottom power reflection coefficient (and the related reflection loss) can be estimated passively by beamforming the naturally occurring marine ambient-noise acoustic field recorded by a vertical line array of hydrophones. However, the reduced array lengths required by small AUV deployment can hinder the process, due to the inherently poor angular resolution. In this dissertation, original data-processing techniques are presented which, by introducing into the processing chain knowledge derived from physics, can improve the performance of short arrays in this particular task. Particularly, the analysis of a model of the ambient-noise spatial coherence function leads to the development of a new proof of the result at the basis of the bottom reflection-loss estimation technique. The proof highlights some shortcomings inherent in the beamforming operation so far used in this technique. A different algorithm is then proposed, which removes the problem achieving improved performance. Furthermore, another technique is presented that uses data from higher frequencies to estimate the noise spatial coherence function at a lower
\end{abstract}


frequency, for sensor spacing values beyond the physical length of the array. By "synthesizing" a longer array, the angular resolution of the bottom-loss estimate can be improved, often making use of data at frequencies above the array design frequency, otherwise not utilized for beamforming. The proposed algorithms are demonstrated both in simulation and on real data acquired during several experimental campaigns. 


\section{Dedication}

This work is dedicated to my family: Francesca, Michelangelo, Maria Luisa, Enrico, Laura, and all the others who have kept showing me their love and support in countless ways, despite the inordinate amount of time we have spent separated, by a long daily commute, or by an ocean and a couple of continents. I am aware of the hard times, fatigue, stress, and sacrifice my working on this dissertation has imposed on all of you. 


\section{Acknowledgments}

I want to thank my adviser Dr. Martin Siderius, who introduced me, first as a collaborator and then as a student, to the NEAR Lab and the field of underwater acoustics. He has offered all the guidance, advice, loyalty, and friendship I much

needed to be successful. He has supported all my graduate ECE studies at Portland State University, and has patiently allowed me to deepen my knowledge of every aspect of the many topics I needed to familiarize myself with, in order to carry out what turned out to be a very significant effort for me. For all this, I am sincerely, deeply grateful.

I gratefully acknowledge the support of the Office of Naval Research Ocean Acoustics Program (ONR-OA Code 3211 and Code 3220A), and the Visiting Researcher Program of the NATO-STO Centre for Maritime Research and Experimentation (CMRE, La Spezia, Italy), which supported me during my work in Italy.

I would also like to thank Drs. Martin Siderius, Lisa M. Zurk, Richard Campbell, and Randy Zelick for serving on my committee.

Being able to take part in measurement campaigns at sea and to work at the CMRE has been a terrific addition to this dissertation and to my professional growth. I am grateful in particular to Dr. Peter L. Nielsen, who has been my supervisor/collaborator in these adventures, is the author or co-author of many of the publications that have stemmed from this work, and has also become a great 
friend of mine and my family's. I want to thank Dr. Yong Min Jiang, who also was my supervisor at the CMRE, for a project not directly related to this dissertation. Finally, I'd like to thank Dr. C.H. Harrison, whose work has provided part of the data and the solid foundations upon which this dissertation rests, and who spent a good amount of time discussing theory with me at the CMRE.

The NEAR Lab has provided a wonderful work environment, where one can truly learn how paramount collaboration is to any significant human effort. I am indebted to all my coworkers at the NEAR Lab for the support they provided, through discussion, friendship, or the mere sharing of our work, which I hope enriched all of us. Thanks in particular to John Gebbie, Sam Henry, Gabe Kniffin, Helen Ou, Joel Paddock, Jorge Quijano, Scott Schecklman, and Elizabeth Thorp Küsel for the time they shared with me. 


\section{Table of Contents}

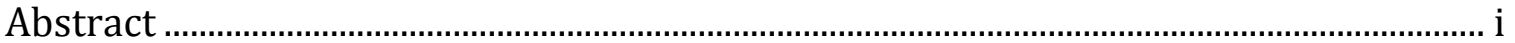

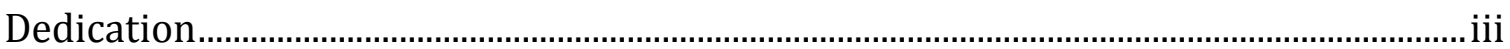

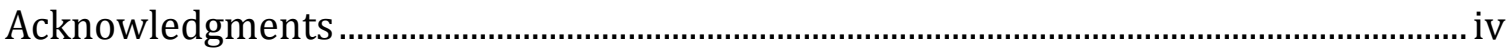

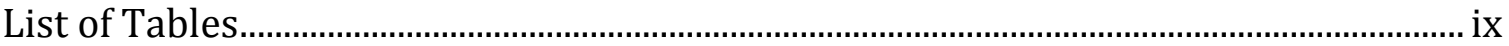

List of Figures

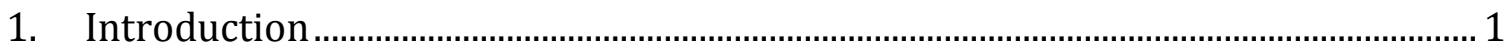

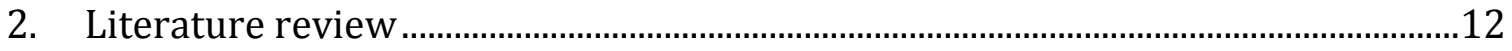

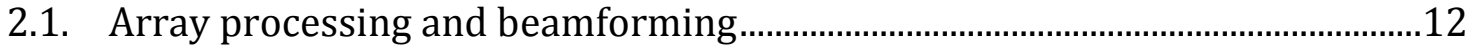

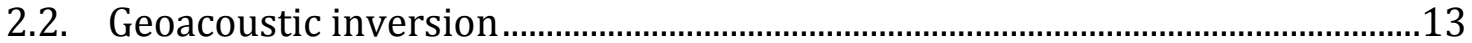

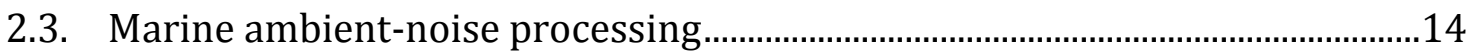

2.4. The marine ambient-noise spatial coherence function .....................................16

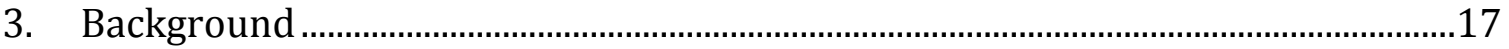

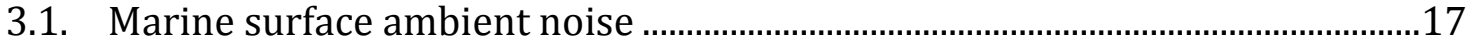

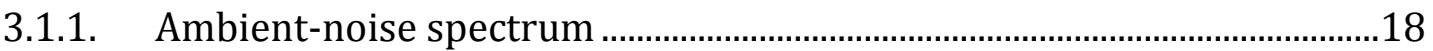

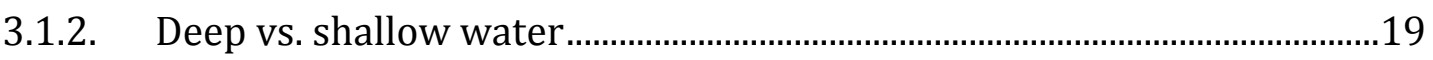

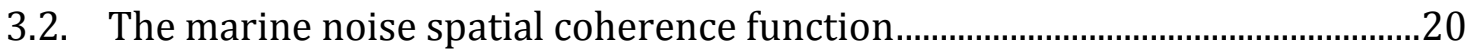

3.3. Array processing and spatial filtering ("beamforming") ........................................21

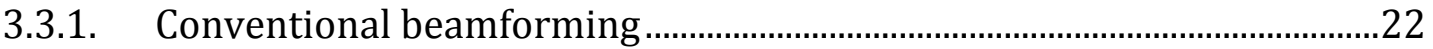

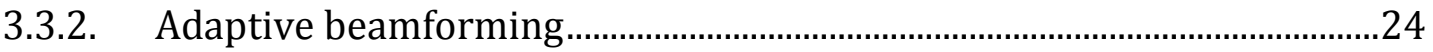

3.4. Passive bottom-loss estimation: Harrison \& Simons' technique ......................25

4. Passive bottom-loss estimation from VLA data ................................................................30

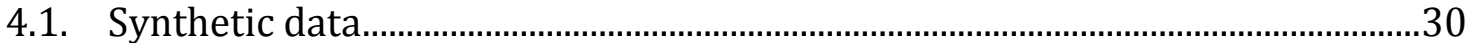

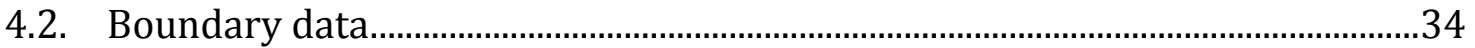

5. Use of adaptive beamforming techniques for bottom-loss estimation...................36

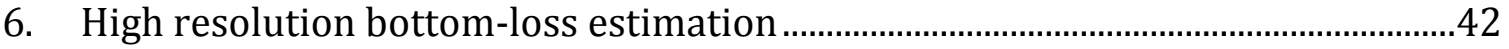

6.1. Derivation of the power reflection coefficient from the noise spatial

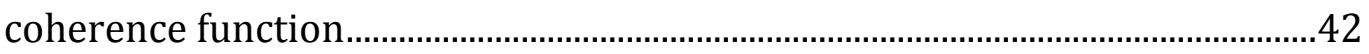

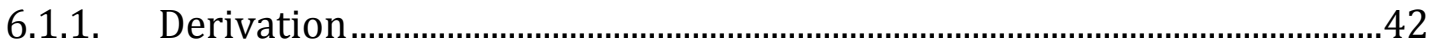


6.1.2. Applicability of the approach ......................................................................4

6.2. Array processing for high resolution bottom-loss estimation...........................50

6.2.1. Technique implementation .....................................................................50

6.2.2. Examples in simple scenarios..................................................................56

6.3. Application to data ........................................................................................5

6.3.1. Application to simulated data......................................................................57

6.3.2. More on the Toeplitz character of the CSD matrix …………………….....62

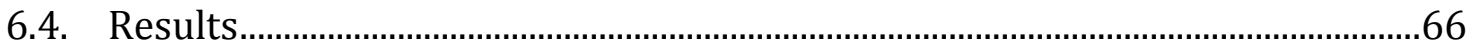

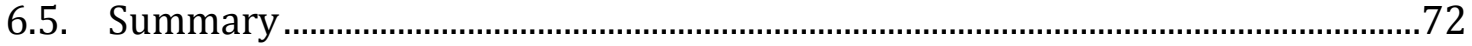

7. Application to arrays suitable for AUV deployment .................................................73

7.1. Results from the GLASS experiment ...................................................................74

7.1.1. GLASS sea trials........................................................................................

7.1.2. Array performance characterization: Sensitivity to tilt and bottom

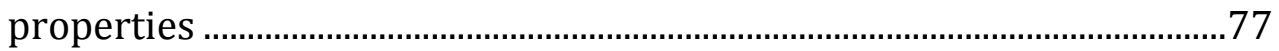

7.1.3. GLASS line-array data processing for bottom-loss estimation ..................80

7.1.4. Use of estimated bottom loss for geoacoustic inversion .............................81

7.1.5. Application of the HR-BL algorithm .............................................................86

7.2. Results from the Recognized Environmental Picture experiment....................87

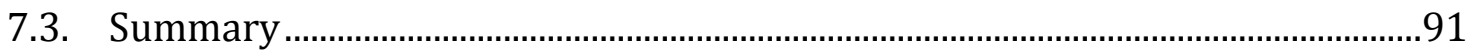

8. Frequency based Coherence-function extension .....................................................93

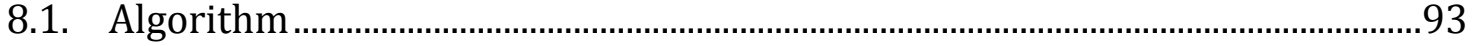

8.2. Modeling and application: Halfspace bottom …………………..........................95

8.3. Considerations on layered bottoms ………………………………………...... 100

8.4. Application to bottom-loss estimation: Simulation .........................................104

8.4.1. Considerations on array configuration and the bandwidth of the estimated bottom loss..................................................................................104

8.4.2. Application to simulated data..................................................................106

8.5. Application to measured data.......................................................................... 113

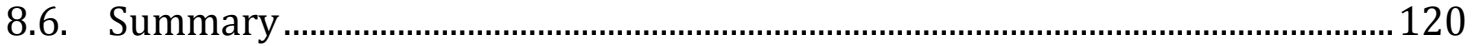

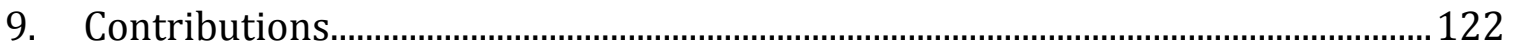

10. Publications/proceedings related to this research ..............................................124 


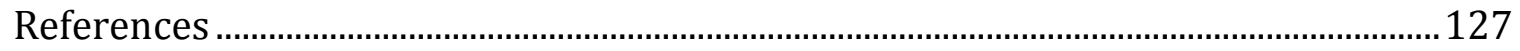




\section{List of Tables}

TABLE I. Water-column and bottom properties for the simulated cases; $\Delta$ is the layer thickness, $\rho$ is the density, $\alpha_{c}$ is the compressional volume attenuation, and $\lambda$ is the wavelength. Shear sound speed and attenuation were set to zero.

TABLE II. Water-column and bottom properties for the simulated cases; $\Delta$ is the layer thickness, $\rho$ is the density, $\alpha_{c}$ is the compressional volume attenuation, and $\lambda$ is the wavelength. Shear sound speed and attenuation were set to zero. Attenuation in water is set by OASN to its lower bound.

TABLE III. Datasets and array basic features.........................................................................67

TABLE IV. Results of GLASS data inversion for two data samples. ................................84

TABLE V. Water-column and bottom properties for the simulated cases; $\Delta$ is the layer thickness, $\rho$ is the density, $\alpha_{c}$ is the compressional volume attenuation, and $\lambda$ is the wavelength

TABLE VI. Datasets and array basic features 


\section{List of Figures}

Figure 1. Definition of coordinate system and geometric quantities. For constant sound speed, the rays are straight lines (hatched), and $\theta_{\mathrm{s}}^{\prime}=\theta_{\mathrm{b}}^{\prime}=\theta_{\mathrm{r}}=\theta$. The solid lines represent ray paths in the presence of a sound-speed profile. The same angle at the receiver $\theta_{\mathrm{r}}$ is considered in both cases.

Figure 2. "Pictorial view" of Harrison and Simons' technique. The noise produced at the surface by the waves is captured by the array when steered towards positive grazing angles. The noise reaching the array from the opposite (negative) angles has undergone an interaction with the bottom. The two "cones" symbolize the ambiguity of the VLA in the azimuth direction. The two lines indicated in real scale represent the length of the arrays used in the Boundary measurement campaigns (more details below).

Figure 3. Bottom loss for the configuration of TABLE I: Computed using Jensen's model (top left) and estimated by applying conventional beamforming to an OASN CSD matrix with 40 (top right), 8 (bottom left), and 5 elements (bottom right). In general, the beamformed estimate appears as a "smoothed" version of the theoretical prediction. This is particularly visible around the critical angle in the top row, where the transition is not as sharp and the detail of the "double" critical angle due to layering (visible in the theoretical prediction above $2500 \mathrm{~Hz}$, between $15^{\circ}$ and $30^{\circ}$ ) is lost. Note the severe deterioration of the estimated bottom loss as the array length decreases.

Figure 4. Estimated bottom loss using data from the Boundary 2003 experiment and an array with the same configuration as that in Section 4.1. The critical-angle transition is not as sharp as one would expect from theory. Some evidence of layering is present at grazing angles beyond $60^{\circ}$, although the striations are quite "smoothed", an effect of the finite beamformer resolution, coupled with the uncertainties in some of the beamforming parameters, such as the relative positioning of the array elements and the sound speed in water.

Figure 5. Bottom loss obtained through Jensen et al. model (a), and from synthetic data produced by OASN for the same bottom type, using CBF (b) and MVDP beamforming (c).

Figure 6. Bottom-loss error $B L_{t h}-B L_{M V D P}$ (left) and main-lobe-width ratio (right) for the MVDP beamformer on the same data as in Figure 5.

Figure 7. Top: CBF beam patterns at $945.5 \mathrm{~Hz}$ at $\vartheta_{\mathrm{s}}=-71^{\circ}$ (hatched) and $\vartheta_{\mathrm{s}}=$ $+71^{\circ}$ (solid). Bottom: MVDP beam patterns at the same frequency and steering angles. The MVDP beam patterns do not display the same symmetry as the CBF ones. When steered towards the bottom, the MVDP beamformer attempts to minimize the contribution from the sources at the surface by lowering the sidelobes 
in the positive grazing-angle region, at the price of a much wider down-looking main lobe.

Figure 8. Correction introduced by the exponential factor from Eq.(31) as a function of grazing angle and sensor depth $\mathrm{h}$ for power attenuation $\mathrm{a}=2.46$. $10^{-4} \mathrm{~m}^{-1}$ and water-column depth $\mathrm{H}=200 \mathrm{~m}$. The correction is relevant especially at very low grazing angles, and can be minimized by positioning the array close to the bottom.

Figure 9. Estimated bottom loss at $2500 \mathrm{~Hz}$ from OASN data for the environment in TABLE I. (a): Theoretical bottom loss ("Theory"), HR-BL processor and CBF using $32 \times 32$ CSD matrices produced by OASN. (b): Same as in (a), but using $16 \times 16$ matrices (the CBF 32 curve is repeated to facilitate a direct comparison). In both cases, when using the same number of physical sensors the HR-BL curve is closer to the theoretical prediction than the $\mathrm{CBF}$ curve over almost the entire angular range. Note the significant degradation of the CBF when moving from 32 to 16 elements, and how the HR-BL 16 curve is very close to the CBF 32 curve.

Figure 10. Estimated bottom loss and error over the frequency range $25-4166 \mathrm{~Hz}$ from OASN data for the environment in TABLE I. The bottom loss is estimated from the same $32 \times 32$ CSD matrix using a Hanning taper and CBF (a) and HR-BL processing (b). The error is computed as the pixel-by-pixel difference in $\mathrm{dB}$ between the bottom loss predicted using the model by Jensen et al. and that estimated by the CBF (c) and the HR-BL processor (d)

Figure 11. Real (top) and imaginary (bottom) parts of the normalized CSD matrices at $2156 \mathrm{~Hz}$, computed from two 5 -minute snapshots (collected about 40 minutes apart) from the BOUNDARY-03 experiment. The matrices in panels c-d appear to be closer to Toeplitz than the matrices in panels $a-b$

Figure 12. Bottom-loss curves: 32 -element CBF vs. HR-BL for the same data as in Figure 11. The drop below zero of the bottom-loss curves visible below $20^{\circ}$ in (a) (corresponding to Figure 11-a,b) is an indication that some violation of the model assumptions is occurring in this 5-minute average. The large oscillations of the HRBL curve are also inconsistent with the curve from data collected about 40 minutes later (b) (corresponding to Figure 11-c,d), where the two techniques compare in a manner analogous to what observed for the OASN simulation (see Figure 9). 64

Figure 13. Beamformer output at $2156 \mathrm{~Hz}$, as a function of steering angle and time, from the dataset used to produce the CSD matrices in Figure 11. A loud interferer appears close to broadside around time 19:25, and approaches the array reaching the closest point around time 19:40, as indicated by the broadening of the angle covered by the interferer. The CSD matrix shown in Figure 11-a,b corresponds to a 5-minute time average centered around time 19:27:30 (when the interferer is quite strongly affecting the array output), while the matrix in Figure 11-c,d is based 
on an average centered around time 20:07:30 (when the interferer's influence is much reduced).

Figure 14. Bottom-loss curves computed from two 5-minute averages (data from the VLA-03 dataset) at $1313 \mathrm{~Hz}(\mathrm{a})$ and $972 \mathrm{~Hz}(\mathrm{~b})$ : Conventional beamforming (CBF) for 32-element and 16-element physical array vs. 16-element HR-BL processor using a Taylor taper with $-30 \mathrm{~dB}$ sidelobe level. In both cases the 16-element HR-BL processor reproduces the features of the 32-element CBF curve more faithfully than the 16-element CBF, and limits the bottom-loss disruption around endfire. 69

Figure 15. Bottom loss curves computed from two 5-minute averages (data from the MFA-03 dataset) at $2000 \mathrm{~Hz}$ (a) and $2250 \mathrm{~Hz}$ (b); processing and naming conventions are the same as in Figure 14.

Figure 16. Bottom loss curves computed from two 5-minute averages (data from the MFA-04 dataset) at $2414 \mathrm{~Hz}$ (a) and $3070 \mathrm{~Hz}$ (b); processing and naming conventions are the same as in Figure 14........................................................................... 71

Figure 17. The GLASS volumetric array — Side and front view. ................................. 75 Figure 18. The eFolaga, with the GLASS volumetric array mounted on its nose,

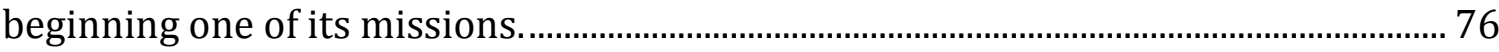

Figure 19. Estimated bottom loss from GLASS'12 (left) and GLASS'13 (right) data. In both cases, the limitations imposed on the estimate by the reduced array length are apparent, when comparing these plots with Figure 4

Figure 20. Beamformed BL from GLASS'12 data (left), and the case resulting from the exhaustive search (right).

Figure 21. Beamformed BL from GLASS'13 data (left), and the case resulting from the exhaustive search (right).

Figure 22. Estimated bottom loss from GLASS12 (left) and GLASS13 (right) using the HR-BL algorithm

Figure 23. SLIVA data: Estimated bottom loss using the full 32-element array.... 89

Figure 24. SLIVA data: Estimated bottom loss using 8 (top), and 16 (bottom) elements of the array, and CBF (left) and HR-BL (right). 90

Figure 25. Halfspace bottom (case HS): Normalized coherence-function real (a) and imaginary (b) part at several frequencies, as a function of sensor spacing z. The markers indicate the positions of sensors number 10 (diamond), 20 (circle), and 40 (square) for an array of spacing $d=0.15 \mathrm{~m}$. 98

Figure 26. Halfspace bottom (case HS): Normalized coherence-function real (a) and imaginary (b) part at the same frequencies as in Figure 25, as a function of the $\mathrm{z} / \lambda$ ratio. The markers indicate the same sensors as in Figure 25, but given the quantity on the horizontal axis, at a lower frequency two consecutive points of a curve are now closer than they are at a higher frequency, and the curve corresponding to a higher frequency reaches higher values on the horizontal axis. Note the almost perfect overlap of the curves. 
Figure 27. Single layer over halfspace (case 1L): Normalized coherence-function real (a) and imaginary (b) part at several frequencies, as a function of the $z / \lambda$ ratio. The markers are positioned as in Figure 26.

Figure 28. Double layer over halfspace (case 2L): Normalized coherence-function real (a) and imaginary (b) part at several frequency values, as a function of the $z / \lambda$ ratio. The markers are positioned as in Figure 26.

Figure 29. Halfspace bottom (case HS): BL predicted using the reflection coefficient given by Jensen et al.'s model (a); BL estimated from OASN data using CBF over 12 (b) and 24 (c) sensors, and BL estimated by HR-BL over 12 sensors extended to 24 by FBE (d).

Figure 30. Single layer over halfspace (case 1L): BL predicted using the reflection coefficient given by Jensen et al.'s model (a); BL estimated from OASN data using CBF over 12 (b) and 24 (c) sensors, and BL estimated by HR-BL over 12 sensors extended to 24 by FBE (d).

Figure 31. Double layer over halfspace (case 2L): BL predicted using the reflection coefficient given by Jensen et al.'s model (a). BL estimated from OASN data using CBF over 12 (b), and 24 (c) sensors, and BL estimated by HR-BL over 12 sensors extended to 24 by FBE (d).

Figure 32. Boundary 2003 MFA data: BL estimated by HR-BL over 32 elements (a), by CBF over 20 (b) and 32 elements (c), and by HR-BL after extending the coherence function estimated from 20 sensors to 32 sensors by FBE (d). 116

Figure 33. Boundary 2003 MFA data: BL estimated by HR-BL over 32 elements (a), by CBF over 20 (b) and 32 elements (c), and by HR-BL after extending the coherence function estimated from 20 sensors to 32 sensors by FBE (d).

Figure 34. Boundary 2003 VLA data: BL estimated by HR-BL over 32 elements (a), by CBF over 20 (b) and 32 elements (c), and by HR-BL after extending the coherence function estimated from 20 sensors to 32 sensors by FBE (d). 118

Figure 35. SLIVA data: BL estimated by HR-BL over 32 elements (a), by CBF over 8 (b) and 32 elements (c), and by HR-BL after extending the coherence function estimated from 8 sensors to 32 sensors by FBE (d). Despite the fact that the length of the array is being increased by a factor of 4 , the high sampling frequency affords the recovery of BL up to the array design frequency. 


\section{Introduction}

SONAR, the underwater equivalent of RADAR, is an important asset for many human activities at sea, and predicting its performance requires knowledge of the acoustic properties of the sea bottom. Traditionally, this knowledge is acquired by means of costly equipment, typically including a ship with crew, a chain of underwater acoustic sensors (hereafter referred to as "array"), whose length varies from several meters to tens of meters, and an artificial acoustic source. Besides being expensive, this methodology has recently begun to raise some concerns, as evidence is being gathered relating the acoustic sources to the periodic stranding of marine mammals on the shore. The research described in this dissertation is part of an effort carried out by the Northwest Electromagnetics and Acoustics Research Laboratory ( NEAR Lab") in collaboration with other institutions to condense the entire system into one small, unmanned submarine, at the same time eliminating the need for the artificial acoustic source by replacing it with the naturally occurring sound of wind flow and breaking waves at the sea surface.

All SONAR systems, whether active or passive, are meant to infer information by exploiting the propagation of sound waves from a source/target to a physically separated receiver. Predicting how the system can perform in a given environment requires the modeling of sound propagation, and particularly in a shallow water environment, the interaction of propagating sound waves with the bottom is an important contribution to the transmission loss. Any propagation model must therefore include accurate information about the acoustic properties of the bottom, 
and, especially for models based on ray tracing, this can be in the form of the power reflection loss as a function of grazing angle and frequency. ${ }^{1}$ The research described in this document focuses in particular on the reflection loss from the bottom (hereafter also referred to as "bottom loss" or "BL"), which is related to the bottom plane wave power reflection coefficient by the equation: ${ }^{2}$

$$
B L\left(\theta_{b}, \omega\right)=-10 \log _{10} R\left(\theta_{b}, \omega\right),
$$

where $R\left(\theta_{b}, \omega\right)$ is the plane-wave power reflection coefficient of the bottom, which in general depends on the wave frequency $\omega$ and grazing angle at the bottom $\theta_{b}$.

In models, the seafloor is usually described as a lossy boundary composed of a number of layers (each characterized by thickness and a set of physical properties) overlaying a rock basement. The presence of layers results in a reflection coefficient that depends on both the incident-wave grazing angle and its frequency. ${ }^{2}$ Unlike the sea surface, the bottom has very little temporal variability, but can have particularly strong spatial variability: Layer thicknesses and composition can vary dramatically within a few hundred meters, ${ }^{3}$ and so do the reflection coefficient and the bottom loss.

The bottom properties are unfortunately very difficult to measure directly in situ (e.g., by analysis of seabed cores), ${ }^{4,5}$ and are typically obtained either from existing environmental databases, or by geoacoustic inversion of measured acoustic data. Existing databases were for the most part developed for modeling propagation in deep water, where the bottom interaction is not as important a contributor to the transmission loss as it is in shallow water. These databases have been shown to 
produce unreliable results, when employed for SONAR transmission loss in shallow water, and the largest source of the error has been identified in the inaccurate information they provide for bottom-loss prediction. ${ }^{6}$ In principle, the necessary spatial resolution for accurate propagation modeling in shallow water can be obtained by geoacoustic inversion. Perhaps the most widely employed geoacoustic inversion methodology so far has been deploying acoustic sources (such as sound projectors or explosive charges, but in some cases exploiting sources of opportunity, such as ship noise) and hydrophone arrays, measuring the acoustic field (sources and arrays could be towed, if an extended area needed to be surveyed), and employing model based matched-field processing to determine the seabed properties by minimizing the mismatch between model predictions and measurement. ${ }^{7-17}$

Besides requiring costly deploying techniques and equipment, this approach is particularly intrusive for the environment, both because of the size of the vehicles and equipment (which also implies energy consumption for transport and operation), and because of the high noise levels introduced by the acoustic sources, which can be deleterious to some applications, as well as to marine life (evidence exists that certain anthropogenic sounds can have a negative impact on marine mammals). ${ }^{18}$

Compared to active systems, passive systems (which only "listen", i.e. exploit acoustic sources already present in the environment) have the advantage of reduced environmental impact, can be easier to deploy, and generate a minimal impact on the overall noise level of the environment in which they operate. Typical applications of these systems have included for decades detection, target localization, and tracking. 
Recently, marine ambient noise naturally generated at the surface (mainly originating from breaking waves and wind) has received increased interest as an acoustic source for passive surveying of the sea bottom. Two passive sea-bottomsurvey systems are Harrison and Simons' technique for bottom-loss estimation ${ }^{19}$ and the passive fathometer. ${ }^{20}$

If one is interested in determining the physical properties of the seabed, Harrison and Simons' technique can be exploited for reliable geoacoustic inversion. ${ }^{21}$ However, the research described in this document focuses in particular on bottom-loss estimation. For this application, one of the main advantages of this technique is that, without the use of an artificial acoustic source, it produces bottom loss directly, as a function of frequency and grazing angle, without requiring data inversion schemes. This is done by processing ambient-noise data collected by a vertical line array of hydrophones through an array-processing technique called beamforming. This technique allows one to use a set of omnidirectional sensors as a directional antenna: Without physically moving the sensors, their outputs can be phased in such a way as to significantly improve the signal-to-noise ratio for waves impinging on the array from a specific direction.

The directional properties of the beamformer vary depending on the geometric arrangement of the sensors. Although three-dimensional arrangements afford the luxury of selectively steering over all three angles in space, the ease of deployment and transportation has made line arrays (where the sensors are positioned along a straight line) often the preferred configuration in underwater acoustics. In the 
particular case of Harrison and Simon's technique, the sensors are positioned along a straight vertical line ("vertical line array", or "VLA" for short), allowing the array to be selectively steered in the elevation-angle direction, which corresponds to the wave's grazing angle on the bottom.

Beamforming is not immune to the inherent limitations of all antennas, which for the purpose of this research affect in particular the angular resolution: All other parameters being equal, the angular resolution improves when the array length (and number of elements) increases. ${ }^{22}$ Since the bottom loss needs to be estimated as a function of grazing angle, limitations in the angular resolution of the array result in limitations on the quality of the estimated bottom loss.

Initially, this technique was conceived and applied to data collected by moored or drifting arrays, the latter deployment affording the possibility of surveying an extensive area as the array is carried by the current. These arrays are usually several meters to several tens of meters long, and have a flexible construction. Such construction allows for easier deployment and transportation, but can negatively impact the performance of high-resolution beamforming techniques (the so called "array mismatch" error), which rely on precise knowledge of the relative positioning of the sensors. ${ }^{23}$

Nowadays, the original implementation of Harrison and Simons' technique is challenged by new developments in operations at sea. The recent interest in Autonomous Underwater Vehicles (AUVs) as marine operational platforms has quickly and dramatically changed the scenario in a number of fields, including seabed 
characterization. AUVs are unmanned submarines, usually of small dimensions, which are programmed with a mission and deployed at sea; they carry out the mission with minimal human supervision, and are then recovered. If the goal of the mission is data collection, the data are usually stored in the memory of the data-acquisition system aboard the vehicle, and downloaded when the vehicle has been recovered.

If it were possible to combine ambient-noise array processing with AUV technology, this would result in an efficient survey tool for seabed characterization. The tool would provide long duration at sea, affording coverage of extended areas with minimal intrusion and impact on the environmental noise levels. Furthermore, it would be cost efficient, because it would minimize the need for both surface vessels, and human interaction during the mission. However, the use of such vehicles imposes design constraints, which vary depending on the size of the AUV, and are mainly related to power consumption, drag, and weight of the whole system. Such constraints affect the propulsion systems of the vehicle - which must be minimally demanding to avoid compromising buoyancy and battery life - but also the equipment that can be deployed on an AUV. This study targets small size AUVs, such as the NEAR-Lab's Slocum glider (Teledyne Webb Research) or the eFolaga (jointly developed by the University of Genova, the CMRE, and GraalTech s.r.l., illustrated to some depth in Section 7). In order for these vehicles to perform bottom characterization, the aforementioned constraints call for:

1. the elimination of the artificial acoustic sources traditionally employed in these studies; 
2. A drastic reduction of the array length;

3. A limited number of array elements (the size and capability of both the battery and the electronics of the acquisition system limit the number of channels that can be processed, as well as the total amount of data that can be stored on board).

Harrison and Simons' passive technique replaces brilliantly the artificial acoustic source with natural marine surface noise generated by waves and wind flow. As far as the array dimensions and number of elements are concerned, the possibility of AUV deployment and potential for elimination of array-mismatch errors have made rigid, short arrays increasingly attractive for a number of applications. However, one of the main obstacles on the way of successful implementation of such a system is the reduced array lengths required by small AUV deployment: Harrison and Simons' technique has proven effective in the $100-5000 \mathrm{~Hz}$ frequency range when employing arrays of lengths between a few meters and several tens of meters. However, small AUVs would require shorter arrays, with a maximum length of $2 \mathrm{~m}$. At these lengths, and at the frequency range indicated above, the inherently poor angular resolution of the beams becomes a matter of concern for the quality of the estimated bottom loss, causing an underestimation of the loss and poor resolution of its grazing angle dependent features. These effects can introduce significant errors when the estimated bottom loss is used directly in propagation models, or in an inversion scheme to estimate geo-acoustic properties of the seabed.24 
Before the start of the research work described in this document, improving the grazing-angle resolution of the estimated bottom loss without increasing the array length had been proposed only by employing adaptive beamforming techniques and, to the author's knowledge, no such attempt had resulted in a peer-reviewed journal publication. Harrison and Baldacci suggested that adaptive beamforming could improve angular resolution in a CMRE technical report, ${ }^{25}$ and so did Siderius and Harrison in 2004, ${ }^{24}$ but a successive CMRE report showed rather unsatisfactory results, ${ }^{26}$ and the idea does not seem to have been pursued further by these researchers.

In a preliminary study based on simulations, Arvelo proposed employing rigid, very short arrays mounted inside the nose of AUVs for bottom-loss estimation. ${ }^{27}$ The vehicles could survey extensive areas in a single mission, collecting data that would subsequently be processed to compute the bottom loss. To overcome the limitations due to the extremely small array aperture, Arvelo limited his study to high frequencies and proposed using aggressive adaptive beamforming techniques to estimate the bottom loss. His conclusion was that such techniques provide reliable results only below the critical angle.

These claims have been investigated also by the author,28,29 by application of adaptive-beamforming techniques to both simulated and measured data (on relatively long arrays), confirming that, consistently with what is available in literature, results cannot be considered satisfactory, at least not beyond the angle regimes indicated by Arvelo. The cause of the problem appears to lie in the nature of 
the adaptive beamforming algorithms, which are designed for detection and/or direction-of-arrival estimation, and do not necessarily yield the accurate estimation of the spatial power spectrum required by bottom-loss estimation.

More recently, the Centre for Maritime Research and Experimentation has started experimenting on the field with AUV-deployable short arrays. They developed a compact, volumetric 8-element array including a $0.4 \mathrm{~m}$ long, 5-element linear array that can be mounted on the nose of an AUV. The acquisition-system test and validation campaign (GLider Acoustics Sensing of Sediments experiment 2012 GLASS'12) produced data (while the vehicle was mounted on a frame moored to the bottom) that showed the potential of the VLA for a rough estimate of basic seabed properties and of the volumetric array for target localization.30,31 In 2014, the Recognized Environmental Picture campaign in the Mediterranean Sea (REP14-MED) employed an 8-element prototype VLA with adjustable spacing, and deployed it together with a 32-element array to be used as reference. The data collected offer a first direct comparison between the performance of the original implementation of Harrison and Simons' technique, and the performance of the new algorithms proposed in this dissertation. Part of the research activities described in this dissertation includes the participation of the author in the GLASS'13 experiment, both during the measurement campaign and the successive data-analysis and processing phase, which resulted in a study of the performance of this array for bottom-loss estimation. The author also participated in the data collection of the acoustic leg of 
the REP14-MED campaign, and the results from the processing of the collected data are included in this dissertation.

The work presented in this document, involving simulation, modeling and data processing/analysis, represents the first investigation of the impact of these particular array configurations on the results obtained by ambient-noise-based bottom survey. The preliminary results show that, in order to successfully migrate Harrison and Simons' technique from longer, moored/drifting arrays to small AUVmounted arrays, improved angular resolution must be achieved, and in this sense adaptive beamforming techniques do not appear to provide reliable results, at least above the critical angle.

In this document, a new derivation of the result at the basis of Harrison and Simons' technique is presented. Furthermore, a technique for increasing the angular resolution of the computed reflection coefficient (and therefore of the derived bottom loss) is demonstrated. The technique, called "high-resolution bottom-loss estimation" (HR-BL), emerges as a natural consequence of the derivation, and exploits the spatial stationarity of the ambient-noise spatial coherence function, a known property of a surface-generated, noise-only field. The higher angular resolution is achieved by removing some inherent limitations of conventional beamforming. ${ }^{32-34}$ The new technique is demonstrated on both synthetic and experimental data. The author also collaborated with another recent study ${ }^{35}$ focused on the vertical coherence function, which proposed employing existing algorithms for the extrapolation of band-limited signals to reconstruct the noise coherence function of a longer array, starting from 
data measured by a shorter array. The extrapolation results appeared promising, but the potential of this technique for bottom-loss estimation has not been investigated yet.

In this dissertation, the idea of overcoming the limitations of short arrays by synthesizing the coherence function of a longer array is treated with the specific purpose of improving the performance of bottom-loss estimation (particularly the angular resolution) through HR-BL. However, instead of applying extrapolation algorithms, the proposed technique, called "frequency based coherence-function extension" (FBE), ${ }^{36,37}$ uses data measured at different frequencies by the physical hydrophones, to approximate the coherence function at the location of the sensors of a longer array. The technique is computationally simple, and makes a more efficient use of the frequency band available to modern acquisition systems, which often extends well beyond the array design frequency. 


\section{Literature review}

This section provides the context for the contributions made in this dissertation. It does so by identifying the current state of research in the fields touched by this work, and pointing out areas that have not been addressed by the open literature. The research described in this document is inherently multidisciplinary and includes topics from the fields of signal/array processing, acoustic-propagation modeling and sea-bottom survey/characterization. Each field is presented separately in the following sections, with special focus on the topics relevant to this dissertation.

\subsection{Array processing and beamforming}

Array processing and beamforming have been well-established applications for a few decades now, and a comprehensive coverage of algorithms and techniques is outside the scope of this document. Excellent, comprehensive references are nowadays available in the form of books: for example, Van Trees ${ }^{38}$ offers thorough coverage of the topic from a communication-theory viewpoint; Johnson and Dudgeon present a more physics-oriented approach;39 some chapters of Manolakis, Ingle and Kogon offer a more concise, yet still surprisingly effective, treatment from a statisticalsignal-processing and adaptive-filtering perspective. ${ }^{40-42}$ Very good review articles are available as well, such as the one by Krim and Viberg. ${ }^{43}$

Part of the work for this dissertation has involved investigating the application of adaptive-beamforming techniques to bottom-loss estimation, for which Cox provides an excellent starting point, illustrating both the optimal minimum-variance 
distortionless response algorithm, and the advantages in terms of robustness of the white-noise gain constraint. ${ }^{23}$ Recursive-least-squares implementations have been developed, to extend the same minimization problem with the introduction of multiple linear constraints and up to a single quadratic constraint. 44,45

\subsection{Geoacoustic inversion}

Characterizing the seafloor for propagation modeling amounts to being able to describe the physical properties of this medium that are relevant to acoustic propagation. This task is of obvious relevance to SONAR operation, and has a wellestablished history, ${ }^{1}$ spread over an impressively large bibliography that cannot be covered in this document. An idea of how complex it is to proceed to this characterization by direct measurement can be gained from the work by Hamilton ${ }^{4}$ and Hamilton and Bachman. ${ }^{5}$ An easier alternative for SONAR prediction is the use of available databases, which were mostly developed and validated for military applications in deep water, the main operating theater during the Cold War. However, when sound-propagation modeling in shallow water environments is required, environmental databases often cannot provide adequately accurate bottom-loss information, which can result in very inaccurate transmission-loss predictions. ${ }^{6}$ The third alternative is geoacoustic inversion of measured acoustic data. In this field, perhaps the most widely employed methodology has so far been deploying acoustic sources (such as sound projectors or explosive charges, but in some cases exploiting sources of opportunity, such as ship noise) and hydrophone arrays, measuring the acoustic field, and employing model-based matched-field processing to determine the 
seabed properties by minimizing the mismatch between model predictions and measurement. A vast literature is available on these methods, which have become more popular as relatively inexpensive computational power has been made available by technological advances. ${ }^{7-17}$ While the examples cited here all pertain to active survey techniques, passive techniques also exist, and are the subject of the next section.

\subsection{Marine ambient-noise processing}

The potential for greatly reduced cost and complexity and, more recently, growing concern about the negative impact on marine life of certain anthropogenic sounds, ${ }^{18}$ have made passive SONAR systems an expanding research field, and this includes applications to seafloor survey. Among these systems, those exploiting marine ambient noise (mainly originating from breaking waves, wind and rain at the surface) as an acoustic source (rather than a disturbance) are of great relevance to this dissertation. In 2002 Harrison and Simons showed that it is possible to estimate the plane-wave power reflection coefficient of the seafloor by comparing the ambientnoise energy harvested by a vertical line array when steered towards the surface and the bottom at opposite elevation angles, ${ }^{19}$ effectively removing for this application the need for artificial acoustic sources. The technique was refined and applied to measured array data in the following years, ${ }^{24,46}$ and extended to investigation of the bottom layering. ${ }^{47-49}$ More recently, Arvelo investigated in simulation the technique's sensitivity to different operative conditions, ${ }^{50}$ and data produced by this technique have been employed in the framework of a Bayesian algorithm for 
geoacoustic inversion. ${ }^{51}$ This technique was also at the origin of another successful noise-processing application known as the passive fathometer, which exploits coherent analysis of vertically steered beams to image seabed layering. ${ }^{20,52-56}$

Recently, the development of Autonomous Underwater Vehicles (AUVs) has reached a stage where they have become a viable technology for a number of applications ranging from military,57 to commercial, 58,59 to research sectors. ${ }^{60}$ Combining ambient-noise-based bottom survey with the versatility ${ }^{61}$ and simplicity of AUVs would produce an attractive, cost-effective bottom-survey system. Due to the nature and size of these vehicles, hydrophone arrays mounted on these systems need to be very compact, and the application of short arrays to bottom characterization has received some interest. ${ }^{24}$ Harrison and Baldacci, ${ }^{25}$ and Siderius and Harrison ${ }^{24}$ suggested that adaptive beamforming could improve angular resolution, but a successive report from CMRE showed rather unsatisfactory results, ${ }^{26}$ and the idea does not seem to have been pursued further by the researchers. A study by Arvelo, ${ }^{27}$ conducted on synthetic data using adaptive-beamforming techniques, showed very limited applicability. Interestingly, adaptive-beamforming techniques have been exploited successfully for the passive fathometer,53,56 whereas application to the problem of bottom reflectivity estimation has not been equally successful ${ }^{26-29}$ (another promising technique for the passive fathometer is practical supergain,62,63 which only applies to arrays steered to endfire). In general, the open literature at this moment lacks a study of the performance and limitations of compact arrays to the specific field of bottom-loss estimation, as well as the presentation of viable 
methodologies for alleviating the effects of their inherent limitations in this application. One of the primary goals of this research work is filling this gap.

\subsection{The marine ambient-noise spatial coherence function}

The marine ambient-noise spatial coherence function plays a fundamental role in beamforming, and has been the subject of extensive theoretical treatment in underwater acoustics, particularly in its relationship to the Green's function. ${ }^{64-76} \mathrm{~A}$ good summary of how this function is modeled for shipping and wind is provided by Hamson. ${ }^{3}$ However, for the research in this dissertation, the wind flow and the breaking waves at the sea surface are the source of choice, and the most useful treatment has been developed for the modeling of passive bottom-survey systems, such as the passive fathometer, $52,53,56$ and Harrison and Simons' technique for bottom-loss estimation. ${ }^{73-75}$ This study also discusses techniques for extrapolating the spatial coherence function, for which a recent application of preexisting bandlimited signal extrapolation techniques ${ }^{79-81}$ has been proposed by Quijano et al..$^{35}$ 


\section{Background}

The research described in this document is inherently multidisciplinary, as it aims at improving the performance of signal- and array-processing algorithms through integration of knowledge provided by physics of the particular problem at hand. Assuming a basic knowledge of underwater acoustics, for each involved discipline this section summarizes the basics and presents the equations that are useful in understanding the rest of the document.

\subsection{Marine surface ambient noise}

[Ambient noise] is what is "left over" after all identifiable noise sources are accounted for. ${ }^{1}$

In signal processing literature and research, the word noise normally indicates a disturbance, an unwanted contribution, or a general background field that competes with a signal. Since the usual purpose is to detect the signal, noise has a negative impact on performance and must be minimized. Urick defines "marine ambient noise" as "that part of the noise background observed with a non-directional hydrophone that is not due to [...] self-noise, or to some identifiable localized source of noise". ${ }^{1}$ The term "self-noise" encompasses cable strumming, waves splashing against the hydrophone, $60 \mathrm{~Hz}$ hum, and all other occasional causes, such as crabs crawling on the sensor. However, in the case of the research illustrated in this dissertation, one component of what Urick calls "noise background" is exploited as a source; as such, it 
constitutes the signal to observe, and its presence is desirable. This section illustrates the basic characteristics of this peculiar acoustic source.

\subsubsection{Ambient-noise spectrum}

The first important trait of the ambient-noise field is that it is generated by different mechanisms, depending on the frequency band and the water-column depth under consideration. The frequency band below $20 \mathrm{~Hz}$ includes noise sources such as tides, waves, and earth seismicity, and is of little interest to this study. The band between $20 \mathrm{~Hz}$ and $500 \mathrm{~Hz}$ appears to be dominated by distant ship traffic, which in deep water can be generated thousands of kilometers away from the measuring hydrophone.

The following band, up to about $30 \mathrm{kHz}$, is the one of greatest relevance to this work. The dominant ambient-noise source in this frequency band is the action of surface waves, through mechanisms whose physics is still not completely understood, such as breaking whitecaps, wind-flow noise (turbulent pressure waves caused by the wind blowing over the rough sea surface), and cavitation. Regardless of the exact origin of the noise, the level of this source has been shown to be correlated with the local wind speed at the surface, over a relatively small area above the measuring hydrophone.

Available deep-water noise spectra include a further band that extends beyond $100 \mathrm{kHz}$, which is not of direct relevance to this study, and where the main source of noise is molecular thermal motion. Other sources of noise at sea are of anthropogenic nature (e.g., explosions, industrial activity in bays and harbors), biological nature 
(such as marine mammals or snapping shrimps), or can be due to a number of intermittent natural phenomena, such as rain, cracking ice, seaquakes, and volcanoes.

\subsubsection{Deep vs. shallow water}

As discussed in the previous section, a number of different sources contribute to the ambient noise field. This section briefly illustrates some generally accepted results describing the influence of the hydrophone depth on the detected noise field from the various sources, providing a higher level of detail on the one that is of more direct interest for the work described in this dissertation: the noise generated at the surface by the action of wind and waves. In the following, the term "shallow water" will refer both to bay and harbor environments, and to coastal waters, such as those on the

continental shelf - the distinction will be made explicitly, when necessary — and "deep water" will identify water-column depths extending beyond the continental shelf.

One of the ways in which the water-column depth influences the noise field is by indirectly "favoring" some sources with respect to others. For instance, in shallow water environments such as bays and harbors the noise sources usually present high temporal and spatial variability: At any given time and location, the strongest contributions to the field in this environment will be provided by a different mix of shipping and industrial noise, wind noise, and biological noise. In contrast, noise from these shallow sources will reach a hydrophone in deep water only as a distant contribution, and its level will depend on the propagation conditions between the hydrophone and the sources. Chapter 7 in Urick's book ${ }^{1}$ provides a summary of 
different studies showing that, in general, the total noise level in the frequency band of interest to this study tends to decrease with increasing depth, with a gradient that is steepest just below the surface. Besides volume attenuation (which varies with frequency), this can also be an effect of bottom topography (deeper locations are "shielded" by surrounding peaks screening out distant sources) or the presence of a surface duct, which traps noise generated at shallow depths in the region below the surface.

The noise level generated by the action of wind and waves at the surface has been found to depend on the wind speed at the surface, directly above the measuring hydrophone. Perhaps more importantly for this study, and quite surprisingly, when the ambient noise is generated only by this source, its level has been found to be independent of hydrophone depth and water depth.

\subsection{The marine noise spatial coherence function}

The spatial coherence function of the pressure field $p(\mathbf{r}, t)$ between two points in space $\mathbf{r}_{1}$ and $\mathbf{r}_{2}$ is defined, in its un-normalized form, as the ensemble average of the product $p\left(\mathbf{r}_{1}, \omega\right) p^{*}\left(\mathbf{r}_{2}, \omega\right)$ :

$$
C_{\omega}\left(\mathbf{r}_{1}, \mathbf{r}_{2}\right) \equiv\left\langle p_{\omega}\left(\mathbf{r}_{1}\right) p_{\omega}^{*}\left(\mathbf{r}_{2}\right)\right\rangle,
$$

where $*$ indicates complex conjugate and $p_{\omega}(\mathbf{r})$ is the coefficient at angular frequency $\omega$ of the Fourier expansion of $p(\mathbf{r}, t)$. It is sometimes useful to refer also to the normalized coherence function $C_{\omega}^{\prime}\left(\mathbf{r}_{i}, \mathbf{r}_{j}\right)$, defined as: ${ }^{76}$ 


$$
C_{\omega}^{\prime}\left(\mathbf{r}_{1}, \mathbf{r}_{2}\right)=\frac{C_{\omega}\left(\mathbf{r}_{1}, \mathbf{r}_{2}\right)}{\sqrt{C_{\omega}\left(\mathbf{r}_{1}, \mathbf{r}_{1}\right) C_{\omega}\left(\mathbf{r}_{2}, \mathbf{r}_{2}\right)}}
$$

and has the advantage of removing from the function the dependence on the noise level at the given frequency.

The noise spatial coherence function, as well as its relationship to the Green's function, has long been the object of studies in underwater acoustics ${ }^{64-76}$ and is of great relevance to the modeling of passive bottom-survey systems such as the passive fathometer 56 and Harrison and Simons' technique for bottom-loss estimation.73,74 However, the bare definitions given in Eq.(2) and Eq.(3) will suffice for the moment, as its fundamental relevance to this study will be clarified by direct references in the following sections.

\subsection{Array processing and spatial filtering ("beamforming")}

Array processing and beamforming have been well-established applications for a few decades now, and a comprehensive summary of algorithms and techniques is outside the scope of this document (among the numerous references that are available, comprehensive texts have been written by Van Trees ${ }^{38}$ and Johnson and Dudgeon, ${ }^{22}$ while a shorter but effective introduction can be found in Manolakis, Ingle and Kogon.) ${ }^{40,42}$ In this section, only the theoretical basics that are necessary for the rest of this document are presented.

The term "array processing" refers to signal-processing techniques applied to a group of signals detected by an array of sensors arranged in a specific geometric 
configuration. When the sensors are equally spaced on a straight line, the array is called a uniform line array (ULA). Just like a finite-impulse-response (FIR) filter processing a uniformly time-sampled signal as input, a ULA spatially samples an impinging wave at equal spatial increments and then processes the signal. A particular application of array processing is beamforming, where the signals from all sensors are linearly combined in order to emphasize signals from a particular direction of arrival, while suppressing signal from any other direction.

\subsubsection{Conventional beamforming}

In the frequency domain, a beamformer produces its output by forming a weighted sum of data (specifically, the Fourier transform of the recorded signals) from the $M$ sensors of the array (for the sake of simplicity, in the following the dependence on frequency and grazing angle will often be dropped in the right-hand side of equations):

$$
y\left(\vartheta_{S}, \omega\right)=\sum_{m=1}^{M} w_{m} p_{m}=\mathbf{w}^{H} \mathbf{p},
$$

In Eq.(4), $H$ denotes the conjugate transpose operation, and $\mathbf{w}\left(\vartheta_{s}, \omega\right)=$ $\left[w_{1}, w_{2}, \ldots, w_{M}\right]^{T}$ is the weight vector for the steering angle $\vartheta_{s}$ ( $T$ denotes the transpose operation). The angle $\vartheta_{s}=0$ corresponds to the array being steered towards broadside (i.e., horizontally for a vertical array), $\vartheta_{s}>0$ towards the surface, and $\vartheta_{s}<0$ towards the bottom. The vector $\mathbf{p}(\omega)=\left[p_{1}(\omega), p_{2}(\omega), \ldots, p_{M}(\omega)\right]^{T}$, where $p_{m}(\omega)=p\left(\mathbf{r}_{m}, \omega\right)=\mathscr{F}\left\{p_{m}(t)\right\}$, represents the data from the $M$ hydrophones in the array $(\mathscr{F}\{\cdot\}$ denotes the Fourier transform). 
Introducing into the picture the stochastic nature of ambient-noise data, the average beam power $B\left(\vartheta_{s}, \omega\right)$ is defined as:

$$
B\left(\vartheta_{S}, \omega\right)=\mathrm{E}\left[\mathbf{w}^{H} \mathbf{p}\left(\mathbf{w}^{H} \mathbf{p}\right)^{H}\right]=\mathbf{w}^{H} \mathrm{E}\left[\mathbf{p} \mathbf{p}^{H}\right] \mathbf{w},
$$

where $E[\cdot]$ denotes expectation. The weight vector $\mathbf{w}\left(\vartheta_{S}, \omega\right)$ can be computed in different ways, depending on the kind of beamformer one wants to implement. For the "conventional beamformer" (CBF), the weight for the $m$-th element in the array is computed as:

$$
w_{m}\left(\vartheta_{S}, \omega\right)=\frac{1}{\sqrt{M}} e^{-i(m-1) \frac{\omega}{c} d \sin \vartheta_{S}},
$$

Where $c$ is the sound speed and $d$ is the array inter-element spacing (assumed constant throughout the array).

The spatial coherence matrix (or cross-spectral-density matrix, hereafter also referred to as "CSD matrix") $\mathbf{C}_{\omega}$ is defined as the expected value of the outer product $\mathbf{p}_{i}(\omega) \mathbf{p}_{i}^{H}(\omega)$ :

$$
\mathbf{C}_{\omega}=\mathrm{E}\left[\mathbf{p} \mathbf{p}^{H}\right]
$$

To make an explicit link to the theory described before, in Eq.(7) element $(i, j)$ in $\mathbf{C}_{\omega}$ is given by the value of the spatial coherence function $C_{\omega}\left(\mathbf{r}_{i}, \mathbf{r}_{j}\right)$ between the two hydrophones [see Eq.(2)]. Typically, in real-world applications an estimate $\widehat{\mathbf{C}}_{\omega}$ of $\mathbf{C}_{\omega}$ is obtained by averaging $\mathbf{p}_{i}(\omega) \mathbf{p}_{i}^{H}(\omega)$ over $K$ data snapshots: 


$$
\hat{\mathbf{C}}_{\omega}=\frac{1}{K} \sum_{i=1}^{K} \mathbf{p}_{i} \mathbf{p}_{i}^{H} .
$$

\subsubsection{Adaptive beamforming}

In the signal-processing literature there is a clear distinction between optimum beamforming techniques, which are mathematically derived assuming perfect knowledge of the statistics of the field being sampled by the array, and their practical implementations, which can only count on estimates of the statistics deduced from measured data. $38,40,42$ To stress this difference, in this section the typical signalprocessing notation is retained, which uses the symbol $\mathbf{R}$ to indicate the covariance matrix of the array output (regardless of the physical nature of the field being measured), and the symbols used in the preceding sections of this document are introduced only when the application pertains specifically to the acoustic noise field.

As shown in Eq.(5), beamforming amounts to computing a set of weights so as to optimize the spatial response of the array on the particular data at hand, with respect to some specified criterion. Among optimum beamforming techniques aimed at maximizing the signal-to-interference-plus-noise ratio at the steering angle, the minimum-variance distortionless-response (MVDR) beamformer computes the weight vector as the solution to the optimization problem:23,40,43

$$
\operatorname{Min}_{\mathbf{w}} \mathbf{w}^{H}\left(\vartheta_{s}, \omega\right) \mathbf{R}_{i+n}(\omega) \mathbf{w}\left(\vartheta_{s}, \omega\right) \text { subject to } \mathbf{w}^{H}\left(\vartheta_{s}, \omega\right) \mathbf{v}\left(\vartheta_{s}, \omega\right)=1 \text {, }
$$


where $\mathbf{R}_{i+n}(\omega)$ is the interference-plus-noise cross spectral-density matrix. The vector $\mathbf{v}\left(\vartheta_{s}, \omega\right)$ is defined as a plane wave of unit amplitude at frequency $\omega$ and incident at an angle $\vartheta_{s}$. Equation (9) shows that the MVDR algorithm tries to maintain unit gain at the steering angle $\vartheta_{s}$, while minimizing the contribution to the total power by noise and interferers coming from directions different from $\vartheta_{s}$. Standard techniques, such as the method of Lagrange multipliers, ${ }^{82}$ can be used to solve the single-constraint minimization problem in Eq. (9), leading to the well-known closed form: $23,40,43$

$$
\mathbf{w}_{M V}\left(\vartheta_{s}, \omega\right)=\frac{\mathbf{R}_{i+n}^{-1} \mathbf{w}}{\mathbf{w}^{H} \mathbf{R}_{i+n}^{-1} \mathbf{w}} .
$$

When processing the marine ambient-noise field, $\mathbf{R}_{i+n}(\omega)$ is not available, and one must resort to algorithms that make use of an estimate of the whole cross-spectral density matrix including signal and possible interference. In this case, the minimumvariance distortionless-processor algorithm (MVDP) is implemented by replacing $\mathbf{R}_{i+n}(\omega)$ with $\widehat{\mathbf{C}}_{\omega}$ in Eq.(9).

\subsection{Passive bottom-loss estimation: Harrison \& Simons' technique}

The seabed bottom loss is an important quantity for predicting transmission loss in the ocean. A passive technique for estimating the bottom loss was first introduced by Harrison and Simons, ${ }^{19}$ and has proven effective in several studies. ${ }^{21,24-49}$ In this technique, the marine ambient-noise field, mainly originating from breaking waves, wind and rain at the surface, is sampled at discrete locations in space by a vertical line array of hydrophones. The data are then beamformed to obtain estimates of the 
power impinging on the array from different angles. The ratio of the averaged noise coming from the seabed to that coming from the surface (at symmetric angles with respect to the horizontal) reveals the loss due to interaction with the seabed, which by definition is the bottom loss.

For a wave front of frequency $\omega$ incident upon the bottom at grazing angle $\theta_{b}>$ 0 (see Figure 1 for the definition of all geometric quantities), the bottom loss is defined as (this equation, already presented in Section 1, is repeated here for convenience):

$$
B L\left(\theta_{b}, \omega\right)=-10 \log _{10} R\left(\theta_{b}, \omega\right) .
$$

Where $R\left(\theta_{b}, \omega\right)$ is the plane-wave power reflection coefficient of the bottom. Harrison and Simons show that the bottom loss can be computed from an estimate $\hat{R}\left(\theta_{b}, \omega\right)$ of the power reflection coefficient obtained from array data as the ratio of the downward and upward beam powers:

$$
\hat{R}\left(\theta_{b}, \omega\right)=\frac{\hat{B}(-\theta, \omega)}{\hat{B}(\theta, \omega)} .
$$

where $\theta=\left|\theta_{r}\right|$ is the absolute value of the angle at the receiver $\theta_{r}$ (i.e., the angle at which a ray reaches the receiver) corresponding to the angle at the bottom $\theta_{b}$. This estimate is then used to replace $E\left[\mathbf{p} \mathbf{p}^{H}\right]$ in Eq.(5), yielding:

$$
\hat{B}\left(\vartheta_{S}, \omega\right)=\mathbf{w}^{H} \hat{\mathbf{C}}_{\omega} \mathbf{w} .
$$


Equation (12) shows that, in bottom-loss estimation, the ratio of the beamformer output power is used to estimate the power ratio of (plane) wave fronts incident upon the array from angles symmetric with respect to the horizontal. A "pictorial view" of the technique is shown in Figure 2.

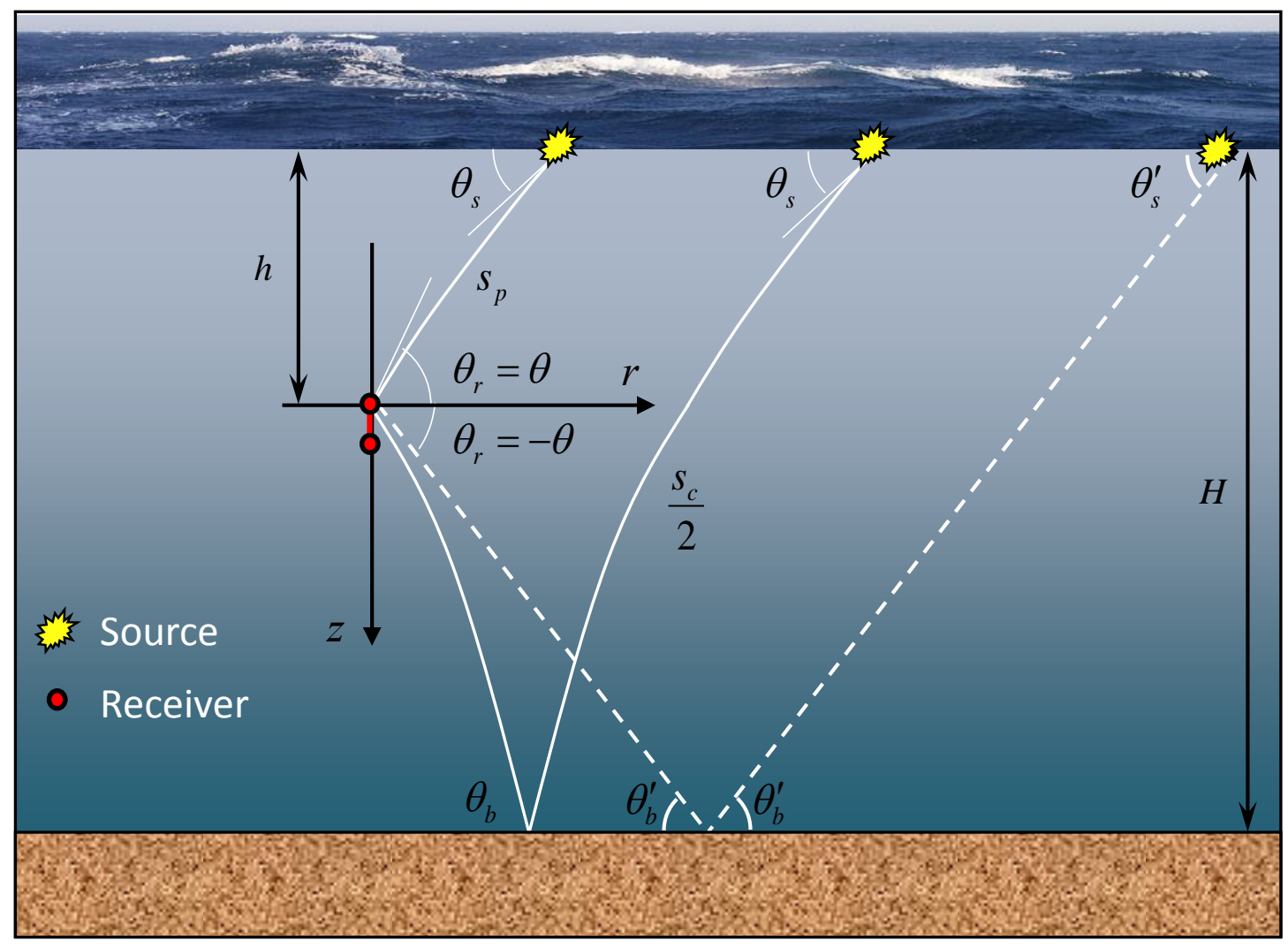

Figure 1. Definition of coordinate system and geometric quantities. For constant sound speed, the rays are straight lines (hatched), and $\theta_{s}^{\prime}=\theta_{b}^{\prime}=\left|\theta_{r}\right|=\theta$. The solid lines represent ray paths in the presence of a sound-speed profile. The same angle at the receiver $\theta_{r}$ is considered in both cases.

The wave propagation angle can be expressed in terms of the vertical wavenumber; for a plane wave propagating at angle $\theta_{0}(e . g$., at the bottom this would be the grazing angle $\theta_{b}$ ), a wave vector $\boldsymbol{\kappa}$ is defined:

$$
\boldsymbol{\kappa} \equiv\left[\begin{array}{ll}
\kappa_{r} & \kappa_{z}
\end{array}\right] \equiv \omega / c\left[-\cos \left(\theta_{0}\right) \quad \sin \left(\theta_{0}\right)\right],
$$


whose Cartesian components $\kappa_{r}$ and $\kappa_{z}$ are the horizontal and vertical wavenumbers, respectively. In the given geometry, and at a given frequency $\omega$, the vertical wavenumber therefore unambiguously identifies the direction of propagation $\theta_{0}$ of the wave front, and Eq.(14) establishes the correspondence between $\theta_{0}$ and $\kappa_{z}$.

The beamformer resolution is the ability of the beamformer to discriminate between wave fronts incident from closely-spaced directions. Adopting the definition based on the Rayleigh criterion, the resolution in wavenumber domain for a linear array is: ${ }^{22}$

$$
\Delta \kappa=2 \pi / L,
$$

where $\Delta \kappa$ is the distance between the two closest values of $\kappa$ that can be resolved and $L=d(M-1)$ is the total length of the array. Equation (15) shows that, for a given sensor spacing, increasing the number of array sensors (and therefore the array length) results in a finer resolution in $\kappa$. 


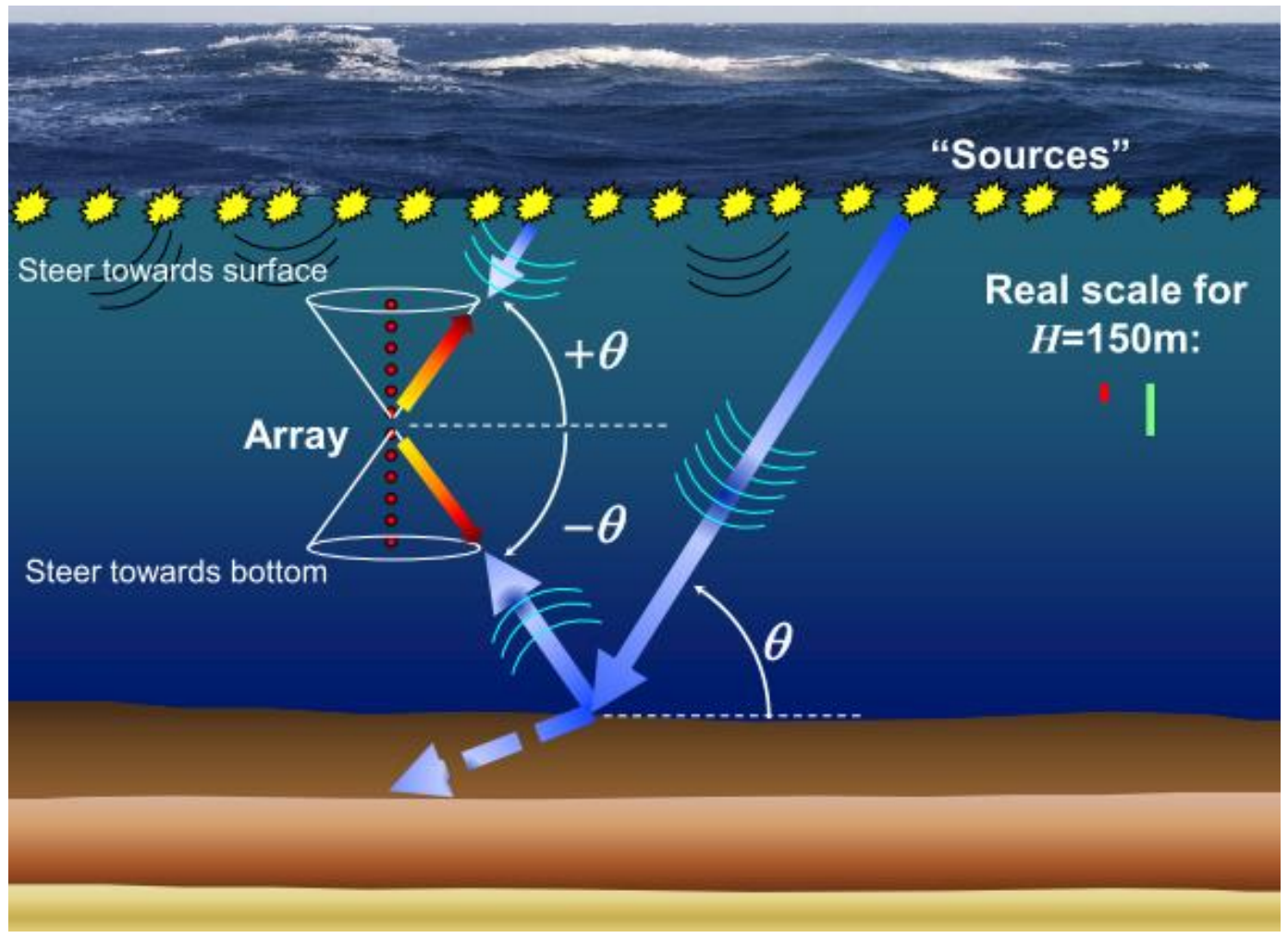

Figure 2. "Pictorial view" of Harrison and Simons' technique. The noise produced at the surface by the waves is captured by the array when steered towards positive grazing angles. The noise reaching the array from the opposite (negative) angles has undergone an interaction with the bottom. The two "cones" symbolize the ambiguity of the VLA in the azimuth direction. The two lines indicated in real scale represent the length of the arrays used in the Boundary measurement campaigns (more details below). 


\section{Passive bottom-loss estimation from VLA data}

Harrison and Simons' technique has been used in a number of studies and does not need to be tested in this research. However, to give the reader an idea of what kind of results it can yield, this section shows some examples of its application to both synthetic and measured data.

\subsection{Synthetic data}

The tool elected to produce synthetic ambient-noise data for this study is OASN, the noise-propagation model of OASES, ${ }^{77}$ which implements a full solution of the wave equation based on wavenumber integration, for horizontally stratified media. For a given bottom type, a theoretical model can be used to compute the corresponding bottom loss as a function of frequency and grazing angle. This can be used as reference in a comparison with the bottom loss estimated by beamforming the OASN CSD matrices; the estimation error is then quantified as the difference between the two values. In this research work it was decided to implement the theoretical model for computing the bottom reflection coefficient described by Jensen et al. ${ }^{2}$

The bottom type used for this test is shown in TABLE I. The array has 32 hydrophones with $0.18 \mathrm{~m}$ spacing. The water-sediment interface is at a depth of $200 \mathrm{~m}$; the sound speed in the water column is constant at $1500 \mathrm{~m} / \mathrm{s}$. The bottom has a $0.75 \mathrm{~m}$ layer resting on a halfspace (see TABLE I for the physical properties). The shallowest hydrophone is at a depth of $180 \mathrm{~m}$. 
Figure 3 shows plots of the bottom loss as a function of frequency and grazing angle. The size, number, and magnitude of the striations visible in the plot depends on the bottom layering and physical properties, and will therefore appear different for different bottom types. In the plot produced using Jensen et al.'s model, the low loss area covering the entire frequency range at the lowest grazing angles is limited to the right by the critical angle. In general, when moving to the plot produced by the beamformer, the limited aperture of the array produces a "smoothed" version of what would be the theoretical bottom-loss plot, since the array's angular resolution cannot be infinitely fine. This is particularly visible around the critical angle, where the transition is not as sharp and the detail of the "double" critical angle due to layering (visible in the theoretical prediction above $2500 \mathrm{~Hz}$, between $20^{\circ}$ and $30^{\circ}$ ) is lost. Furthermore, the array fails to measure bottom loss at very low frequencies because, as indicated by Eq.(14) and Eq.(15), the angular resolution becomes poorer at lower frequencies. This means that the array is harvesting energy from a very broad range of grazing angles, which tends to bring the ratio in Eq.(12) close to one, causing the result of Eq.(11) (i.e. the estimate of the bottom loss) to approach zero. For all the plots estimated using an array, the vertical axis only covers a frequency range up to the array "design frequency": Above such frequency, which is a function of the ratio of the array spacing to the wavelength, the array resolution drops abruptly, as evidenced by the low-loss (blue) area in the upper right corner of the estimatedbottom-loss plot. 
What Figure 3 shows, which is fundamental for the remainder of this dissertation, is that reducing the array length has the effect of magnifying the "smoothing" effect due to the array's finite aperture. The deterioration of the bottom-loss estimate becomes more severe as the array length becomes smaller, in comparison to the signal wavelength.

TABLE I. Water-column and bottom properties for the simulated cases; $\Delta$ is the layer thickness, $\rho$ is the density, $\alpha_{c}$ is the compressional volume attenuation, and $\lambda$ is the wavelength. Shear sound speed and attenuation were set to zero.

\begin{tabular}{ccccc}
\hline & $\Delta(\mathrm{m})$ & $c_{p}(\mathrm{~m} / \mathrm{s})$ & $\rho\left(\mathrm{kg} / \mathrm{m}^{3}\right)$ & $\alpha_{c}(\mathrm{~dB} / \lambda)$ \\
\hline Water & 170 & 1500 & 1000 & $10^{-4}$ \\
Sediment & 0.50 & 1565 & 1500 & 0.2 \\
Halfspace & $\infty$ & 1800 & 2000 & 0.5 \\
\hline \hline
\end{tabular}



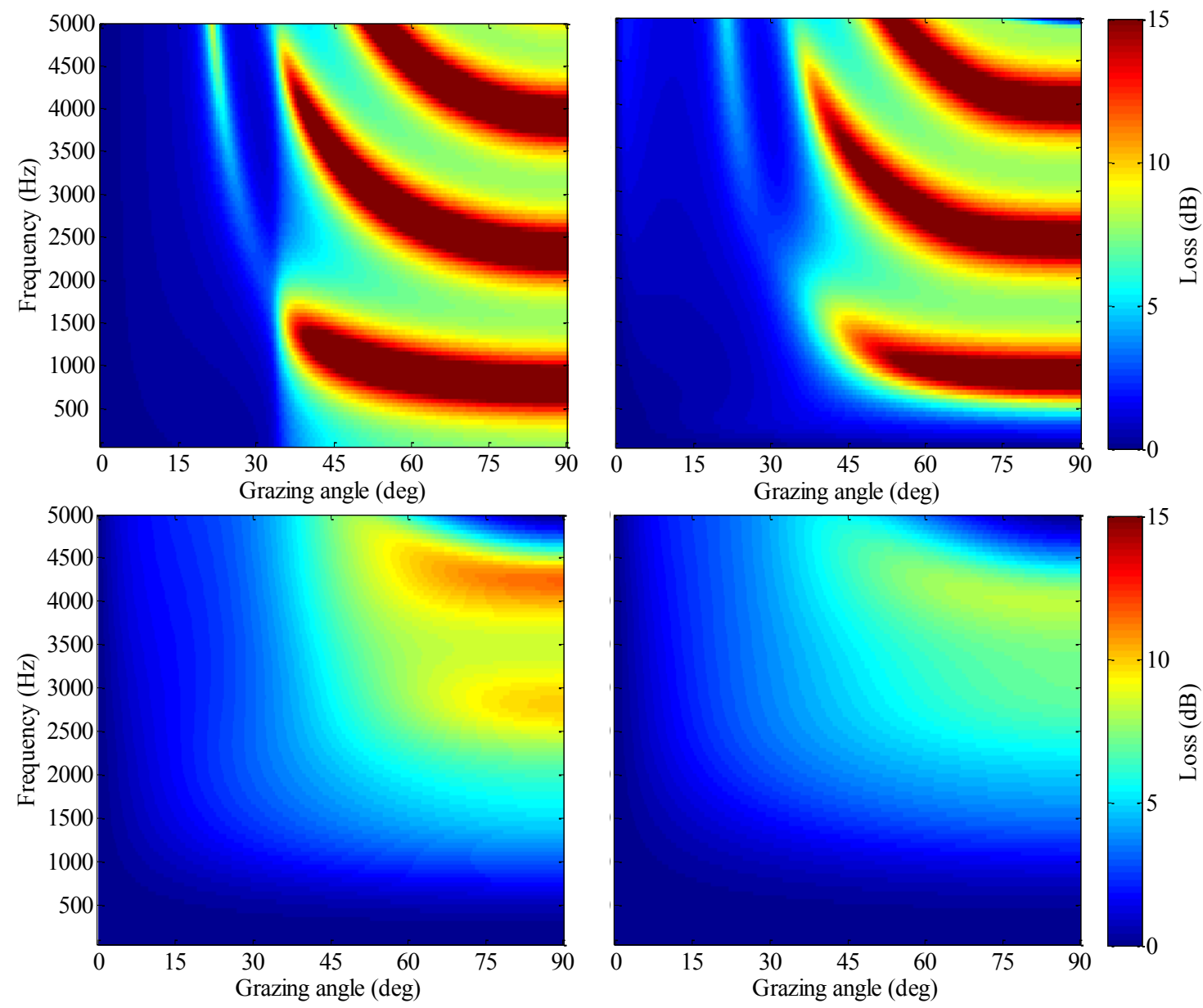

Figure 3. Bottom loss for the configuration of TABLE I: Computed using Jensen's model (top left) and estimated by applying conventional beamforming to an OASN CSD matrix with 40 (top right), 8 (bottom left), and 5 elements (bottom right). In general, the beamformed estimate appears as a "smoothed" version of the theoretical prediction. This is particularly visible around the critical angle in the top row, where the transition is not as sharp and the detail of the "double" critical angle due to layering (visible in the theoretical prediction above $2500 \mathrm{~Hz}$, between $15^{\circ}$ and $30^{\circ}$ ) is lost. Note the severe deterioration of the estimated bottom loss as the array length decreases. 


\subsection{Boundary data}

The research performed for this study has included applying Harrison and Simons' technique to a number of different datasets, some of which have been studied for years by the scientific community (data from the Boundary 2003, and the Boundary 2004 experiments), others are more recent (GLASS 2012 and GLASS 2013 experiments) and others are presented for the first time (REP14-MED experiment). More details on these experiments and datasets are provided in Sections 6-8; this section presents one example of the results, to give the reader an idea of what they look like, compared to the theoretical and simulated results shown above.

In the real world, the data are collected as sampled time histories by each hydrophone in the array. The data are then moved to the frequency domain through a Fast Fourier transform, averaged as indicated in Eq.(8) and beamformed according to Eq.(13). Figure 4 shows the result of beamforming on a data segment from the Boundary 2003 experiment. Compared to the simulated results, these show in particular poorer resolution of the interference striations and a much smoother transition at the critical angle. 


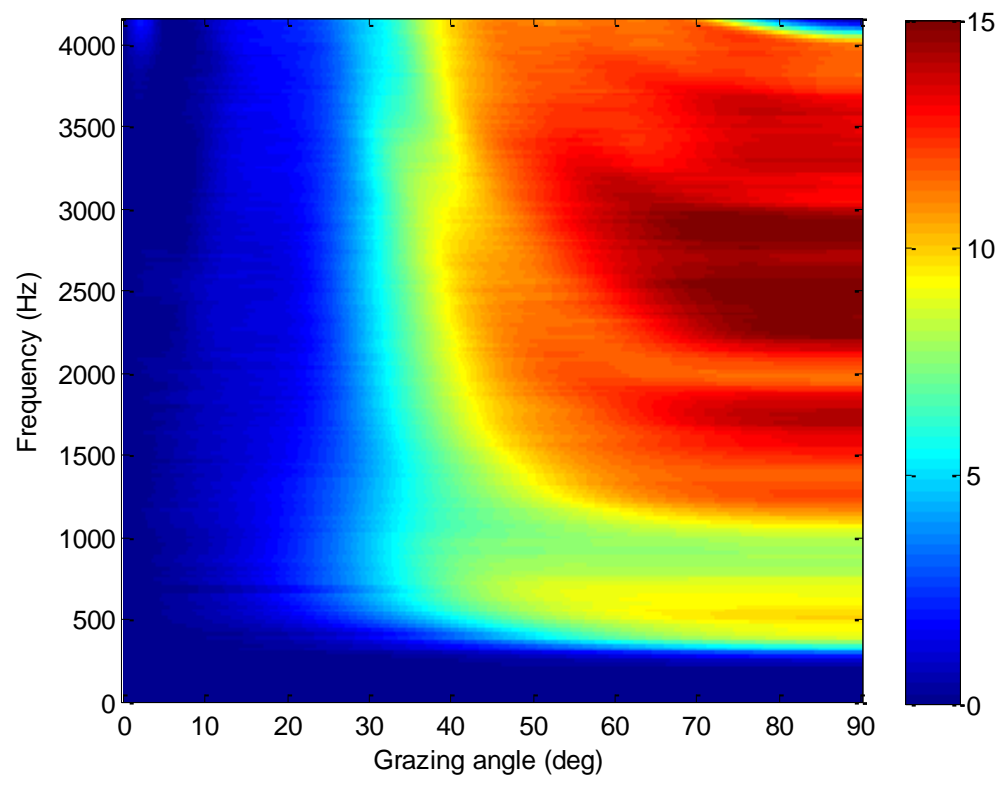

Figure 4. Estimated bottom loss using data from the Boundary 2003 experiment and an array with the same configuration as that in Section 4.1. The critical-angle transition is not as sharp as one would expect from theory. Some evidence of layering is present at grazing angles beyond $60^{\circ}$, although the striations are quite "smoothed", an effect of the finite beamformer resolution, coupled with the uncertainties in some of the beamforming parameters, such as the relative positioning of the array elements and the sound speed in water. 


\section{Use of adaptive beamforming techniques for bottom-loss estimation}

As previously explained in Section 3, Harrison and Simons' passive technique for estimating the bottom loss is based on beamforming of the marine ambient-noise field using a vertical line array. Although studies available in literature and based on measured data (as opposed to simulations) so far have only made use of conventional beamforming, some authors have suggested that adaptive beamforming techniques might improve the BL estimation. ${ }^{24,27,50}$ This has found partial confirmation for grazing angles below the critical angle in a study involving simulated data. ${ }^{27}$ The minimum-variance distortionless processor is a beamforming algorithm that has proven to achieve excellent performance in direction-of-arrival estimation. In this section, the application of this adaptive beamformer for BL estimation is explored by applying it to both simulated and measured data.

The BL plots in Figure 5 are obtained by computing $\hat{R}\left(\theta_{b}, \omega\right)$ with Jensen's model ${ }^{2}$ (a) and by beamforming a noise field produced by $\operatorname{OASN}^{77}$ (b and c), all for the same bottom type. Figure 5(c), in particular, shows that the MVDP beamformer performs significantly worse than the CBF in this application, generating artifacts such as an area of significant BL disruption around endfire, and disruptive striations running approximately perpendicularly to the BL ridges. 


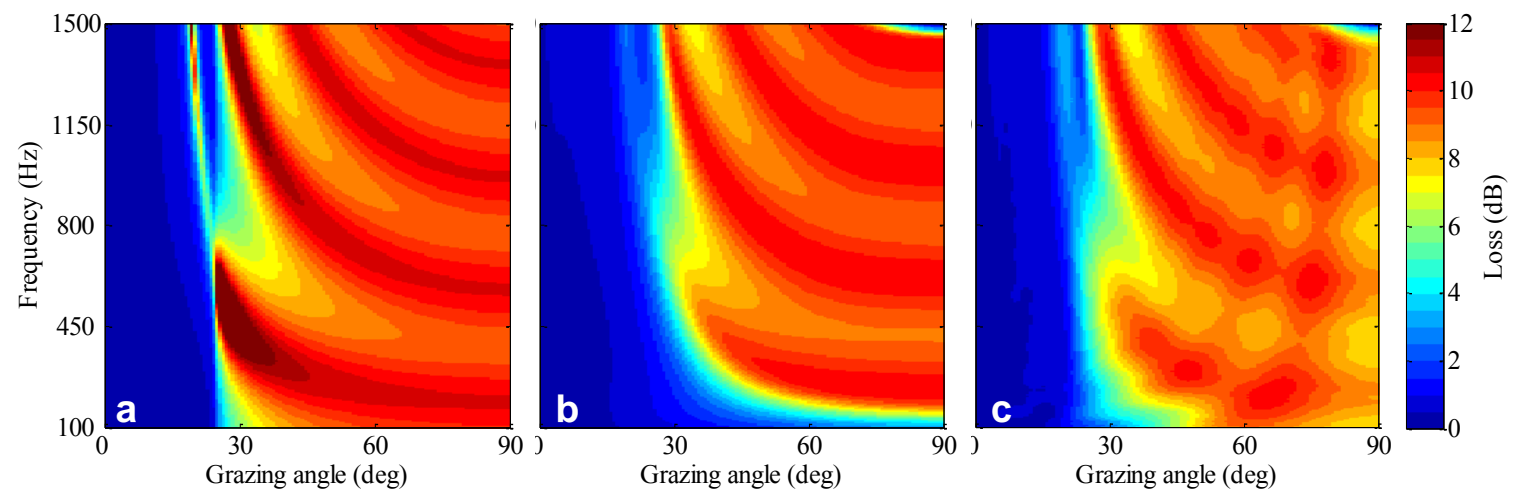

Figure 5. Bottom loss obtained through Jensen et al. model (a), and from synthetic data produced by OASN for the same bottom type, using CBF (b) and MVDP beamforming (c).
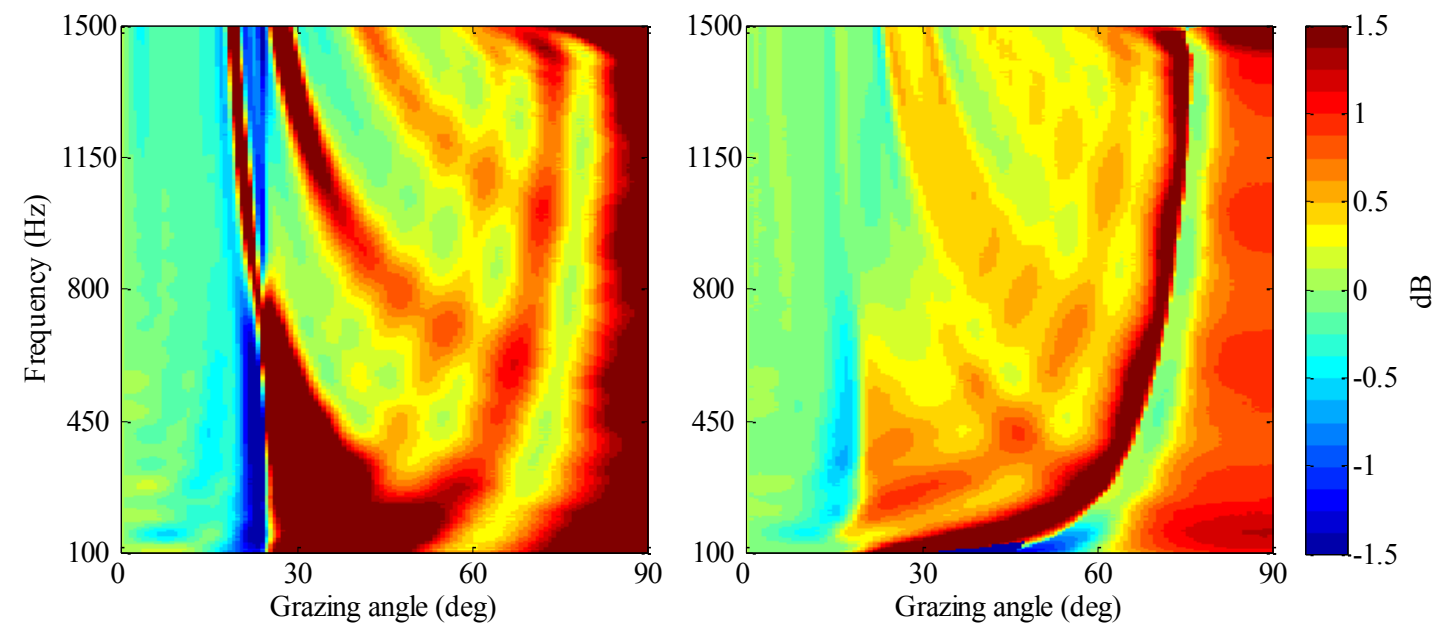

Figure 6. Bottom-loss error $B L_{t h}-B L_{M V D P}$ (left) and main-lobe-width ratio (right) for the MVDP beamformer on the same data as in Figure 5.

The disruption can be quantified by comparison with the predicted BL obtained from the theoretical model: Figure 6 shows the difference between the theoretical $\left(B L_{t h}\right)$ and the MVDP $\left(B L_{M V D P}\right)$ results as $B L_{t h}-B L_{M V D P}$ [i.e., the difference between (a) and (c) in Figure 5]. The disruption is particularly significant across the whole frequency spectrum at endfire and around the critical angle, and at low frequencies 
above the critical angle; the disruptive striations are clearly visible in the whole region to the right of the critical angle. Since these are synthetic data, the position of the array elements is known without ambiguity, and array mismatch can be ruled out as a possible cause of the artifacts. Furthermore, diagonal loading was used to stabilize the cross spectral density matrices with respect to inversion, to avoid effects due to numerical instability. Finally, the artifacts are absent in the CBF results, both for real and synthetic data. All these considerations suggest that the artifacts should be regarded as a consequence of the MVDP algorithm itself.

As shown in Eq.(6), the CBF weight vector depends only on the array configuration and the sound speed in the medium, whereas the MVDP weight vector in Eq.(10) includes properties of the acoustic field through the cross spectral density matrix $\mathbf{R}_{i+n}$. As shown in Figure 7(a), the data-independent CBF beam pattern has a "regular" shape, with sidelobes whose level decreases with distance from the steering angle, and when the array is steered towards two symmetric angles $+\vartheta_{s}$ and $-\vartheta_{s}$, the two beam patterns are perfectly symmetric around the horizontal grazing angle $\theta=0$. When comparing CBF and MVDP beam patterns, it is apparent that the adaptive nature of the MVDP beamformer destroys this symmetry, and yields beam patterns of more complex shape [Figure 7 (b)]. The MVDP beamformer, when steered towards the bottom, attempts to reduce the contribution of the surface sources to the total power by lowering the sidelobes that point towards the surface (positive grazingangle values). But in the case of Figure 7 (b) $\left(\vartheta_{s}=-71^{\circ}, f=945.5 \mathrm{~Hz}\right)$, the price to pay for this optimization is a wide main lobe. When steering towards the surface, the 
beamformer can afford higher sidelobes towards the bottom — because, due to the $\mathrm{BL}$, the contribution of bottom bounces to the total power is significantly lower and maintain a narrow main lobe at the steering angle.
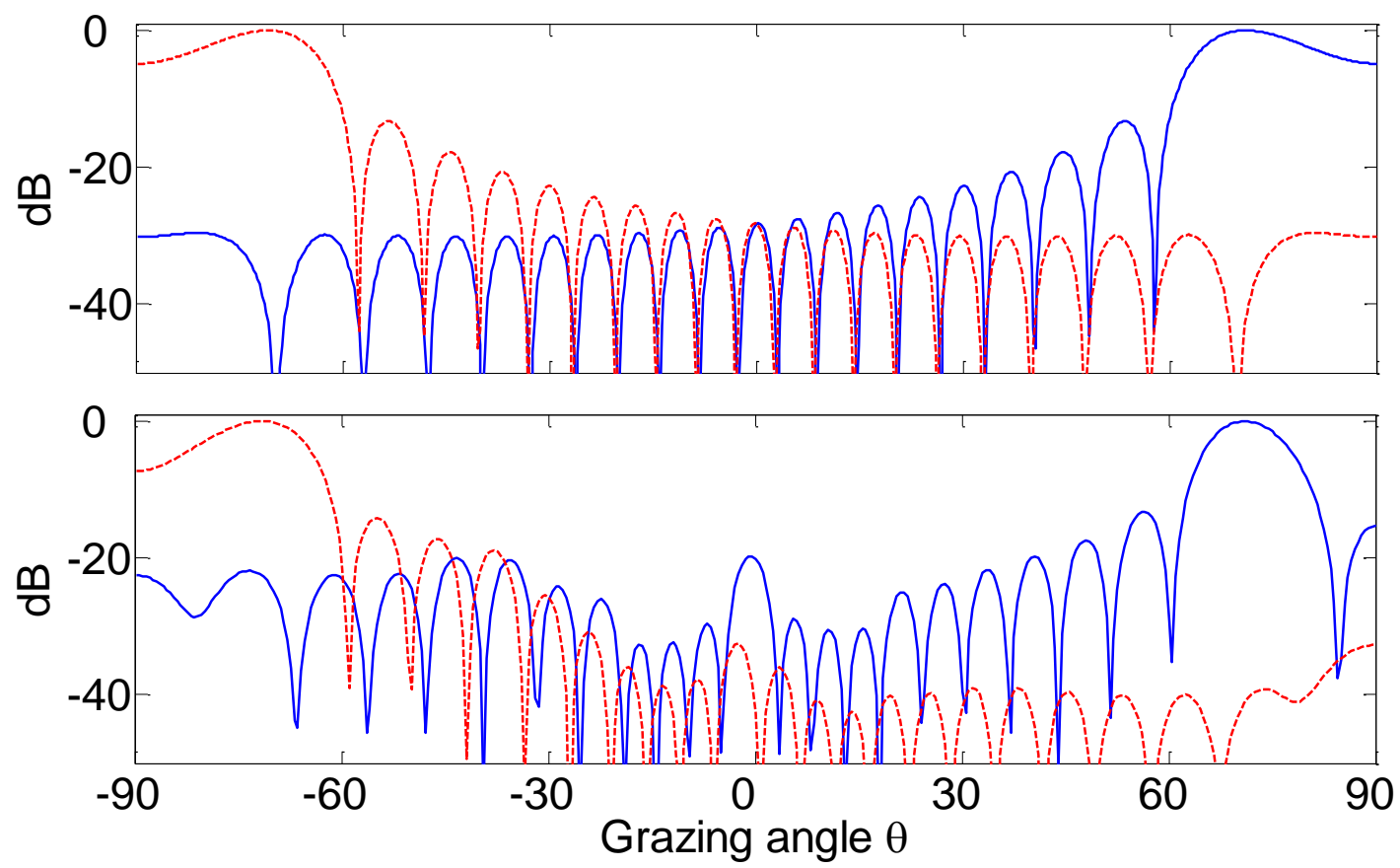

Figure 7. Top: $\mathrm{CBF}$ beam patterns at $945.5 \mathrm{~Hz}$ at $\vartheta_{s}=-71^{\circ}$ (hatched) and $\vartheta_{s}=$ $+71^{\circ}$ (solid). Bottom: MVDP beam patterns at the same frequency and steering angles. The MVDP beam patterns do not display the same symmetry as the CBF ones. When steered towards the bottom, the MVDP beamformer attempts to minimize the contribution from the sources at the surface by lowering the sidelobes in the positive grazing-angle region, at the price of a much wider down-looking main lobe.

It seems reasonable to make the hypothesis that this main-lobe asymmetry could affect the ratio in Eq.(12) to the point that it no longer represents a correct estimate of the directional power ratio, therefore ruining the BL estimate. To quantify the influence of this parameter, in this work the main-lobe width $W$ is defined as the full width at half maximum - i.e., the difference in grazing-angle value of the two $-6 \mathrm{~dB}$ 
points that are closest (on opposite sides) to the main-lobe maximum in the beam pattern plot.

Figure 6 (b) shows on a dB scale the ratio of the width of the down-looking main lobe $\left(W_{-}\right)$to the width of the up-looking main lobe $\left(W_{+}\right)$, for each pair of steering angles $\pm \vartheta_{S}$, as a function of frequency and grazing angle; the orientation of the axes is the same as in the BL plots. By comparison to the BL-error plot in Figure 6(a), it is apparent that both plots show ridges in the same positions where the disruptive striations appear in the MVDP BL plot [see Figure 5(c)]. Particularly, a darker area at endfire, and a strong ridge running from about $75^{\circ}$ and $f=1.5 \mathrm{kHz}$ to about $20^{\circ}$ and $f=100 \mathrm{~Hz}$ in the width-ratio plot clearly correspond to similar features in the BLerror plot, and to BL disruption in the MVDP BL pattern of Figure 7.

The same calculations and analyses were performed on other data sets, both measured and simulated, verifying that the artifacts shown above and their correspondence with main lobe asymmetry are not specific to the particular data set shown here. These results provide empirical evidence that the main lobe asymmetry plays an important role in the disruptive striations observed in MVDP-estimated BL plots.

In conclusion, beamforming of the underwater noise field using the MVDP algorithm can result in inaccurate estimation of the BL. Errors around the critical angle and in the low-frequency region above the critical angle are typical artifacts of this BL-estimation technique, due to the broadening of the beams induced by the finite length of the array. The MVDP algorithm introduces other errors, which 
concentrate particularly around endfire and along striations running approximately perpendicularly to the BL ridges in the BL-pattern plots. The locations of these errors correspond to locations at which the main lobes of the beam patterns steered towards symmetric directions show markedly different widths. More specifically, the MVDP algorithm can produce down-looking main lobes that are significantly wider than the corresponding up-looking main lobes. This appears to be a condition induced by the MVDP algorithm in the attempt to minimize contribution to the total power by the sources on the surface, when steering towards the bottom.

These considerations, together with the little support to the idea found in literature, indicate that the application of adaptive beamforming algorithms to Harrison and Simons' technique presents significant challenges. The reason for this appears to lie in the "mismatch" between the task these algorithms are designed for (direction-of-arrival estimation) and the purpose they should serve in BL estimation (accurate estimation of the spatial power spectrum). Algorithms such as the MVDP are designed to suppress interferers covering a very narrow range in angle of incidence, and whose level is above the signal that needs to be detected. However, in BL estimation, when steering towards the bottom, the surface ambient noise appears to the MVDP algorithm as an "interferer" with ample spatial coverage and a level comparable to the "signal" that should be detected (i.e., the reflection from the bottom). The two scenarios are virtually one the opposite of the other, and therefore the unpredictable behavior of the MVDP should not be surprising. 


\section{High resolution bottom-loss estimation}

In the previous section, it has been shown that the application of Harrison and Simons' technique to arrays whose length is short, compared to the signal wavelength, presents new, significant challenges, related in particular to the low angular resolution of the array. Besides increasing the array length, no other method for improving angular resolution in this particular application is available in literature (the application of adaptive beamforming techniques has not been proved to be a viable solution above the critical angle.) ${ }^{27-29}$ In this section, an original derivation is presented that shows how, under some reasonable conditions, the bottom reflection coefficient can be obtained from the array spatial coherence function without conventional beamforming. The prerequisite is that the array CSD matrix be (approximately) Toeplitz, which is a known property for a surfacegenerated, noise-only field. Furthermore, a technique for increasing the angular resolution of the computed reflection coefficient (and therefore of the derived bottom loss) is demonstrated. The technique emerges as a natural consequence of the derivation, and is demonstrated on both synthetic and experimental data.

\subsection{Derivation of the power reflection coefficient from the noise spatial coherence function}

\subsubsection{Derivation}

The un-normalized spatial coherence function and its relationship to the CSD matrix have already been introduced in Eq.(2) and Eq.(7) (for the sake of brevity, "unnormalized" will be omitted in the remainder of this document, but the spatial 
coherence function should always be considered in this form, unless otherwise specified.) This section presents the derivation in the frequency-wavenumber domain of a formula for computing the power reflection coefficient from the coherence function of the surface-generated marine noise field as recorded by the array.

Using a ray-based approach Harrison derived a formula for the spatial coherence function of surface-generated noise in the ocean, which for the case of two hydrophones joined by a perfectly vertical line and separated by a distance $z$ is written (see Figure 1 for the definition of the coordinate system and all geometric quantities):64,74

$$
\begin{aligned}
C_{\omega}(z)= & \int_{0}^{\pi / 2} \frac{2 \pi \sin \theta_{s} \cos \theta_{r}}{1-R_{s}\left(\theta_{s}\right) R\left(\theta_{b}\right) e^{-a s_{c}\left(\theta_{r}\right)}} \\
& \left\{e^{i(\omega / c) z \sin \theta_{r}} e^{-a s_{p}}+R\left(\theta_{b}\right) e^{-i(\omega / c) z \sin \theta_{r}} e^{-a\left[s_{c}\left(\theta_{r}\right)-s_{p}\left(\theta_{r}\right)\right]}\right\} d \theta_{\mathrm{r}} .
\end{aligned}
$$

In Eq.(16), $C_{\omega}(z)$ introduces a more compact notation $C_{\omega}(z) \equiv C_{z}\left(\mathbf{r}_{1}, \mathbf{r}_{2}\right)$, where it is assumed that $\mathbf{r}_{1}=(0,0)$ and $\mathbf{r}_{2}=(0, z)-i . e$. the hydrophone pair is assumed to be aligned with the $z$ axis, whose origin is at the depth of hydrophone 1. Furthermore, $\theta_{r}, \theta_{s}$, and $\theta_{b}$ are the ray angles at the receiver, the surface, and the bottom; $s_{c}$ and $s_{p}$ are the complete and partial ray-path lengths (specifically, $s_{c}$ is the length of a surface-bottom-surface ray path), whose dependence on $\theta_{r}$ is determined by the sound-speed profile in the water column, $c$ is the sound speed at the receivers, and $R$ and $R_{S}$ are the bottom and surface power reflection coefficients. In general, besides the ray angle, the reflection coefficients are also a function of frequency, but for the 
sake of simplicity this dependence will not be indicated explicitly. Note that $a$ is the power attenuation per unit length along the ray path. The model assumes that the hydrophones are "close", so that a single ray path and sound speed can be defined for the sensor pair.

Since $\theta_{r}$ is limited in Eq.(16) to non-negative values, by defining $\theta \equiv\left|\theta_{r}\right|$ and using Snell's law:

$$
\begin{aligned}
\theta_{s} & \equiv \sin ^{-1}\left(\frac{c}{c_{s}} \sin \theta\right) \\
\theta_{b} & \equiv \sin ^{-1}\left(\frac{c}{c_{b}} \sin \theta\right),
\end{aligned}
$$

(where $c_{s}$ and $c_{b}$ indicate the sound speed at the surface and bottom, respectively) the equation can be rewritten as a function of the sole variable $\theta$ :

$$
\begin{aligned}
C_{\omega}(z)= & \int_{0}^{\pi / 2} \frac{2 \pi\left(c / c_{s}\right) \sin \theta \cos \theta}{1-R_{s}(\theta) R(\theta) e^{-a s_{c}(\theta)}} \\
& \left\{e^{i(\omega / c) z \sin \theta} e^{-a s_{p}}+R(\theta) e^{-i(\omega / c) z \sin \theta} e^{-a\left[s_{c}(\theta)-s_{p}(\theta)\right]}\right\} d \theta .
\end{aligned}
$$

Now let:

$$
k \equiv \frac{\omega}{2 \pi c} \sin \theta_{r}=\frac{\sin \theta_{r}}{\lambda},
$$

where $\lambda$ is the signal wavelength; then $0<\theta_{r}=\sin ^{-1}(\lambda k)<\pi / 2$ gives the bounds $0<k<1 / \lambda$ [note that Eq.(19) defines $k$ as a scaled vertical wavenumber at the receiver: $k=\kappa_{z} / 2 \pi$.] By substituting $\theta \equiv \sin ^{-1}(\lambda|k|)$ into Eq.(17) and Eq.(18), and defining:

$$
\frac{\widetilde{G}(k)}{\lambda} \equiv \frac{2 \pi\left(c / c_{s}\right) \lambda|k|}{1-R_{s}(k) R(k) e^{-a \widetilde{s}_{c}(k)}},
$$


Harrison's equation can be rewritten as:

$$
\begin{aligned}
C_{\omega}(z) & =\int_{0}^{1 / \lambda} \widetilde{G}(k) e^{-a \widetilde{s}_{p}(k)} e^{i 2 \pi z k} d k \\
& +\int_{0}^{1 / \lambda} \widetilde{G}(k) R(k) e^{-a\left[\widetilde{s}_{c}(k)-\widetilde{s}_{p}(k)\right]} e^{-i 2 \pi z k} d k
\end{aligned}
$$

Now by letting:

$$
\begin{aligned}
& \widetilde{G}_{1}(k) \equiv \widetilde{G}(k) e^{-a \widetilde{s}_{p}(k)} ; \\
& \widetilde{G}_{2}(k) \equiv \widetilde{G}(k) e^{-a\left[\widetilde{s}_{c}(k)-\widetilde{s}_{p}(k)\right]},
\end{aligned}
$$

and by introducing the generalized rectangle function:

$$
\Pi(x) \equiv \begin{cases}0 \text { for } & |x|>1 / 2 \\ 1 \text { for } & |x| \leq 1 / 2\end{cases}
$$

equation (21)can be rewritten as:

$$
\begin{aligned}
C_{\omega}(z)= & \int_{0}^{1 / \lambda} \widetilde{G}_{1}(k) e^{i 2 \pi z k} d k+\int_{0}^{1 / \lambda} \widetilde{G}_{2}(k) R(k) e^{-i 2 \pi z k} d k \\
= & \int_{-\infty}^{\infty} \widetilde{G}_{1}(k) \Pi(\lambda k-1 / 2) e^{i 2 \pi z k} d k \\
& +\int_{-\infty}^{\infty} \widetilde{G}_{2}(k) R(k) \Pi(\lambda k-1 / 2) e^{-i 2 \pi z k} d k \\
= & \mathscr{f F}^{-1}\left\{\widetilde{G}_{1}(k) \Pi(\lambda k-1 / 2)\right\}+\mathcal{F}\left\{\widetilde{G}_{2}(k) R(k) \Pi(\lambda k-1 / 2)\right\} .
\end{aligned}
$$

Equation (24) states that the two addends can be expressed as a direct and an inverse Fourier transform between the $z$ and the $k$ domains. Taking the Fourier transform of both sides yields:

$$
\begin{aligned}
\mathscr{F}\left\{C_{\omega}(z)\right\}= & \mathscr{F}^{\mathcal{F}}\left\{\mathscr{F}^{-1}\left\{\widetilde{G}_{1}(k) \Pi(\lambda k-1 / 2)\right\}\right\} \\
& +\mathscr{J}^{\mathscr{F}}\left\{\mathscr{F}\left\{\widetilde{G}_{2}(k) R(k) \Pi(\lambda k-1 / 2)\right\}\right\} \\
\equiv & \mathcal{F}^{+}(k)+\mathcal{F}^{-}(k) .
\end{aligned}
$$


The first addend in Eq.(25) reduces to the argument of the inner inverse Fourier transform:

$$
\mathcal{F}^{+}(k) \equiv \mathscr{F}^{f}\left\{\mathscr{F}^{-1}\left\{\widetilde{G}_{1}(k) \Pi(\lambda k-1 / 2)\right\}\right\}=\widetilde{G}_{1}(k) \Pi(\lambda k-1 / 2),
$$

whereas the second addend, by applying the duality property of the Fourier transform, yields:

$$
\begin{aligned}
\mathcal{F}^{-}(k) & \equiv \mathscr{F}\left\{\mathscr{F}\left\{\widetilde{G}_{2}(k) R(k) \Pi(\lambda k-1 / 2)\right\}\right\} \\
& =\widetilde{G}_{2}(-k) R(-k) \Pi(\lambda k+1 / 2) .
\end{aligned}
$$

Equations (25)-(27) show that $\mathscr{F}\left\{\mathrm{C}_{\omega}(\mathrm{z})\right\}$, the $k$-spectrum of the coherence function, is split into a portion $\mathcal{F}^{+}(k)$, which is nonzero only for positive $k$ values, and a portion $\mathcal{F}^{-}(k)$, which is nonzero only for negative $k$ values. $R(k)$ can now be computed as the ratio:

$$
\begin{aligned}
R(k) & =\frac{\mathcal{F}^{-}(-k)}{\mathcal{F}^{+}(k)} \frac{\widetilde{G}_{1}(k)}{\widetilde{G}_{2}(k)} \\
& =\frac{\mathcal{F}^{-}(-k)}{\mathcal{F}^{+}(k)} e^{-a\left[2 \widetilde{s}_{p}(k) \widetilde{s}_{c}(k)\right]}, \quad k \in\left[\begin{array}{ll}
0 & \frac{1}{\lambda}
\end{array}\right],
\end{aligned}
$$

where, recalling that both $\tilde{G}(k)$ and $R(k)$ are even functions of $k, \mathcal{F}^{-}(-k)$ is given by:

$$
\mathcal{F}^{-}(-k)=\widetilde{G}_{2}(k) R(k) \Pi(\lambda k-1 / 2)
$$

Note that, because of the rectangle functions in $\mathcal{F}^{+}(k)$ and $\mathcal{F}^{-}(k)$, the power reflection coefficient $R(k)$ is defined only for $\in\left[\begin{array}{ll}0 & 1 / \lambda\end{array}\right]$, i.e. $\theta_{r} \in\left[\begin{array}{ll}0 & \pi / 2\end{array}\right]$, which are the integration limits in Eq.(16). Since negative values of $k$ correspond to $\theta_{r}<0$ in Eq.(19) - i.e. waves reaching the hydrophones after reflection from the bottom - 
and positive values of $k$ correspond to $\theta_{r}>0-$ i.e. reflection from the surface - the result in Eq.(28) is reminiscent of the method for estimating $R$ described by Harrison and Simons, ${ }^{2}$ but it provides an explicit account of the influence of the volume attenuation on the estimate of $R$ that the energy-flux argument in the original reference did not have. Furthermore, Eq.(28) is the theoretical basis of the improvedresolution BL-estimation algorithm illustrated in Section 6.2.

If volume attenuation in the water column is neglected, Eq.(20), Eq.(22), and Eq.(28) can be simplified by dropping the exponential factors. For a lossy medium, Eq.(28) shows that the ratio of the two halves of $\left\{\mathrm{C}_{\omega}(\mathrm{z})\right\}$ must be corrected by the additional exponential factor, which takes into account volume attenuation along the partial and complete ray paths. In general, the exact form of $\tilde{s}_{c}$ and $\tilde{s}_{p}$ depends on the sound-speed profile, and given the definition in Eq.(11), this factor adds to the bottom loss an excess of $10 \log _{10}\left\{\exp \left[2 a \tilde{s}_{p}(k)-a \tilde{s}_{c}(k)\right]\right\}$, which needs to be subtracted when estimating the bottom loss using Harrison and Simons' method. However, Eq.(28) also shows that this correction can be minimized by positioning the array close to the bottom (a similar conclusion was reached by Arvelo, ${ }^{50}$ although in the context of a different derivation): In this case the approximation $2 \tilde{s}_{p} \approx \tilde{s}_{c}$ can be considered valid for most grazing angles (see Figure 1), and the value of the exponential term approaches one.

For the special case of an isospeed water column of depth $H$, assuming the hydrophone pair is at depth $h$, the complete and partial ray-path lengths are: 


$$
\begin{aligned}
& \widetilde{s}_{c}(k) \equiv \frac{2 H}{\lambda|k|} \\
& \widetilde{s}_{p}(k) \equiv \frac{h}{\lambda|k|},
\end{aligned}
$$

and Eq.(28) becomes:

$$
R(k)=\frac{\mathcal{F}^{-}(-k)}{\mathcal{F}^{+}(k)} e^{-\frac{2 a}{\lambda|k|}(h-H)}, \quad k \in\left[\begin{array}{ll}
0 & \frac{1}{\lambda}
\end{array}\right]
$$

As an example, the correction to the bottom loss that one should apply when using Harrison an Simons' technique, computed from the exponential in Eq.(31), is plotted in Figure 8 as a function of grazing angle in a water column of depth $H=200 \mathrm{~m}$, for different values of the sensor depth $h$ and $a=2.46 \cdot 10^{-4} \mathrm{~m}^{-1}$ (a value typical for a frequency of $10 \mathrm{kHz}$ ). Since the sensor depth can vary between zero and $H$, when $h=$ $H$ the exponential factor is identically equal to one, whereas when $h<H$ the plot shows that the correction is relevant especially at very low grazing angles, and is minimized when the array is close to the bottom. 


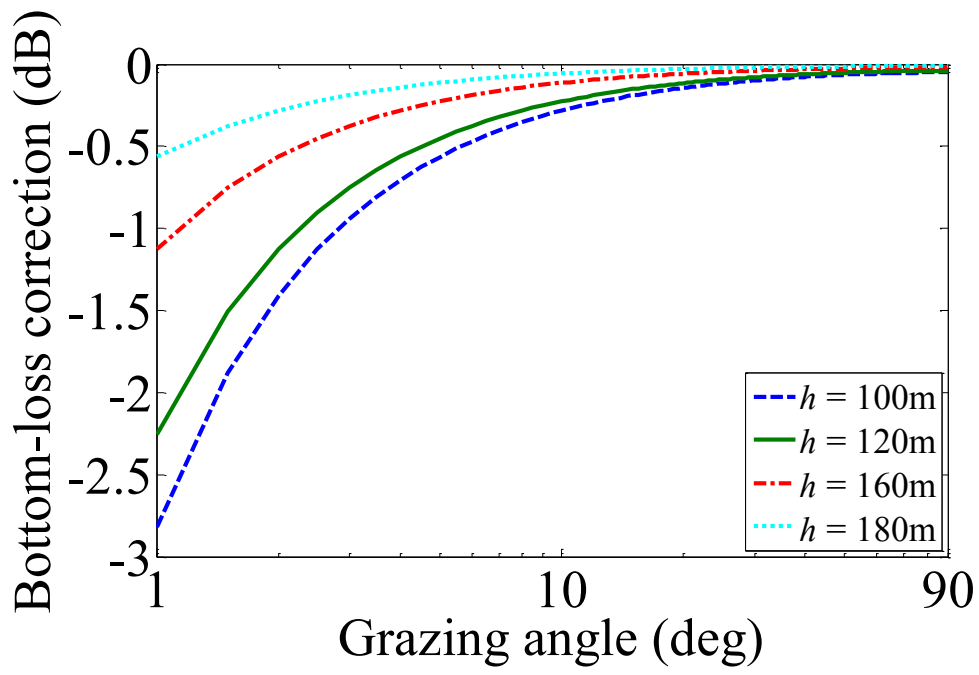

Figure 8. Correction introduced by the exponential factor from Eq.(31) as a function of grazing angle and sensor depth $h$ for power attenuation $a=2.46$. $10^{-4} \mathrm{~m}^{-1}$ and water-column depth $H=200 \mathrm{~m}$. The correction is relevant especially at very low grazing angles, and can be minimized by positioning the array close to the bottom.

\subsubsection{Applicability of the approach}

The conditions under which the results shown above apply are determined by the assumptions underlying Eq.(16). For this study, the most important assumption is that the acoustic field be generated by surface noise: The derivation does not make allowances for sources of a different kind. In the real world, this implies being able to acquire data when the surface noise is sufficiently high and shipping interference is negligible. The derivation also assumes that the spacing between the hydrophones whose data are being correlated is small enough to guarantee that the angle at the receiver for a given ray is the same for both hydrophones. This condition is usually well approximated for vertical line arrays, especially those used for beamforming. In the derivation, the hydrophones are also assumed to be joined by a perfectly vertical 
line. When this is not the case (e.g., for a tilted array), the accuracy in the estimate of $R(k)$ deteriorates, but this is not investigated in this study.

Finally, a correction is required in Eq.(16) in the proximity of boundaries. Harrison ${ }^{64}$ shows that such correction can be safely neglected at distances from the boundaries of the order of $\lambda / \sin \theta_{c}$ (where $\theta_{c}$ is the critical angle), which, e.g., corresponds to about $1.5 \mathrm{~m}$ for $\theta_{c}=20^{\circ}$ and a $3 \mathrm{kHz}$ signal. When these conditions are met, CSD matrices produced by Eq.(16) result in estimates of $R(k)$ in excellent agreement with those produced using OASN, the noise-propagation module of OASES,77 which implements a full wave solution based on wavenumber integration for horizontally stratified media.

\subsection{Array processing for high resolution bottom-loss estimation}

\subsubsection{Technique implementation}

When working with array data, measurements are only available at the locations of the sensors, so the coherence function $C_{\omega}(z)$ is sampled at integer multiples of $d$ along the $z$ axis, and its Fourier transform in Eq.(25) must be interpreted as a discrete Fourier transform (DFT). The resolution of the DFT in spatial wavenumber $k$ increases with the number of samples available to the transform, i.e. with the number of array elements. This translates into better estimation of the seabed bottom loss, but it comes at the price of physically increasing the array length. An alternative approach is proposed here, which is based on the idea of exploiting the physical properties of $C_{\omega}(z)$ before applying the DFT. 
In order to explain the technique, a first important consideration is the dependence of the coherence function on the hydrophone-pair depth $h$. This dependence appears implicitly in Eq.(16) in the difference between $s_{c}$ and $s_{p}$, and its effect on the bottom reflection coefficient is quantified by the exponential correction factor in Eq.(28). This correction can become important at very shallow grazing angles, but this effect can be minimized by positioning the array close to the bottom.

When this is added to the conditions outlined in Section 6.1.2, the noise coherence function between two hydrophones depends primarily on the distance between the hydrophones, rather than their absolute position in the water column. In other words, equally-spaced hydrophones have the same coherence function, regardless of their position in the array. This spatial stationarity of the marine ambient-noise field has been theoretically proved and verified against experimental data for both deep 66,75 and shallow water (at sufficient distance from the waveguide boundaries).64,69 Harrison's ray treatment estimated that the spatial coherence function becomes weakly dependent on sensor depth at a distance from the waveguide boundaries of the order of a few wavelengths. ${ }^{64}$

For the CSD matrix $\mathbf{C}_{\omega}$, the spatial stationarity of $C_{\omega}(z)$ implies that $C_{\omega}\left(\mathbf{r}_{i}, \mathbf{r}_{j}\right)=$ $C_{\omega}\left(\mathbf{r}_{l}, \mathbf{r}_{m}\right)$ when $(i-j)=(l-m)$, i.e., besides being Hermitian by construction, the matrix is Toeplitz. Finally, the spatial stationarity also implies that $C_{\omega}(z)$ is (approximately) conjugate symmetric:

$$
C_{\omega}(-z) \approx C_{\omega}^{*}(z) .
$$


The Toeplitz structure of $\mathbf{C}_{\omega}$ implies that all the relevant information is contained in its first row. The elements in each of the diagonals parallel to the main diagonal are all equal, and all correspond to the same value of $z$. However, the number of (repeated) elements in each diagonal decreases linearly with increasing $z$. This has important consequences on the quality of the $\mathrm{BL}$, when $\mathrm{CBF}$ is used for its estimation, as in Harrison and Simons' technique.

The following is a key proof, in order to understand the advantages provided by the algorithm for BL estimation presented in this section. The proof shows that, when $\mathrm{CBF}$ is implemented as a matrix product, as in Eq.(13), is has an effect equivalent to applying a triangular shading window to the spatial coherence function.

The simple case of a 4-element array provides a starting point, from which the expressions for a general, $M$-element array are then derived. The weight vector for a 4-element array is:

$$
\mathbf{w}\left(\vartheta_{s}, \omega\right)=\left[\begin{array}{llll}
w_{1} & w_{2} & w_{3} & w_{4}
\end{array}\right]^{T}
$$

where the expression for the individual weight $w_{m}$ was given earlier in Eq.(6). Furthermore, by exploiting its Toeplitz character, the CSD matrix can be expressed as:

$$
\mathbf{C}=\left[\begin{array}{llll}
c_{1,1} & c_{1,2} & c_{1,3} & c_{1,4} \\
c_{2,1} & c_{2,2} & c_{2,3} & c_{2,4} \\
c_{3,1} & c_{3,2} & c_{3,3} & c_{3,4} \\
c_{4,1} & c_{4,2} & c_{4,3} & c_{4,4}
\end{array}\right] \equiv\left[\begin{array}{llll}
c_{0} & c_{1} & c_{2} & c_{3} \\
c_{1}^{*} & c_{0} & c_{1} & c_{2} \\
c_{2}^{*} & c_{1}^{*} & c_{0} & c_{1} \\
c_{3}^{*} & c_{2}^{*} & c_{1}^{*} & c_{0}
\end{array}\right]
$$


where the subscript of each element indicates the magnitude (in units of $d$ ) of the spacing between the two sensors whose coherence is given by the element itself. It is important, for this proof, to note that in Eq.(34), elements corresponding to negative spacing values - i.e., according to the convention established in Section 6.1.1, elements whose row index is greater than their column index — are easily identified by the complex conjugate sign accompanying them. The average beam power can then be computed explicitly as:

$$
\begin{aligned}
& \mathbf{w}^{\mathrm{H}} \mathbf{C} \mathbf{w}=w_{1} w_{1}^{*} c_{0}+w_{1} w_{2}^{*} c_{1}^{*}+w_{1} w_{3}^{*} c_{2}^{*}+w_{1} w_{4}^{*} c_{3}^{*} \\
& +w_{2} w_{1}^{*} c_{1}+w_{2} w_{2}^{*} c_{0}+w_{2} w_{3}^{*} c_{1}^{*}+w_{2} w_{4}^{*} c_{2}^{*} \\
& +w_{3} w_{1}^{*} c_{2}+w_{3} w_{2}^{*} c_{1}+w_{3} w_{3}^{*} c_{0}+w_{3} w_{4}^{*} c_{1}^{*} \\
& +w_{4} w_{1}^{*} c_{3}+w_{4} w_{2}^{*} c_{2}+w_{4} w_{3}^{*} c_{1}+w_{4} w_{4}^{*} c_{0}
\end{aligned}
$$

and, by regrouping:

$$
\begin{aligned}
& \mathbf{w}^{\mathrm{H}} \mathbf{C} \mathbf{w}=\left(w_{1} w_{1}^{*}+w_{2} w_{2}^{*}+w_{3} w_{3}^{*}+w_{4} w_{4}^{*}\right) c_{0} \\
& +\left(w_{2} w_{1}^{*}+w_{3} w_{2}^{*}+w_{4} w_{3}^{*}\right) c_{1}+\left(w_{1} w_{2}^{*}+w_{2} w_{3}^{*}+w_{3} w_{4}^{*}\right) c_{1}^{*} \\
& +\left(w_{3} w_{1}^{*}+w_{4} w_{2}^{*}\right) c_{2}+\left(w_{1} w_{3}^{*}+w_{2} w_{4}^{*}\right) c_{2}^{*} \\
& +w_{4} w_{1}^{*} c_{3}+w_{1} w_{4}^{*} c_{3}^{*}
\end{aligned}
$$

It is now necessary to introduce a more explicit expression for the weight products in Eq.(36). For CBF, by using the substitution $\delta=(\omega / c) d \sin \theta$, the expression for the weights $w_{m}$ given in Eq.(6) becomes:

$$
w_{m}=\frac{1}{\sqrt{M}} e^{-i(m-1) \delta}
$$


and, with some algebra, the products in Eq.(36) can be written for a generic sensorindex $m$ as:

$$
\begin{gathered}
w_{m} w_{m}^{*}=\frac{1}{M} \\
w_{m} w_{m+n}^{*}=\frac{1}{M} e^{i n \delta} \\
w_{m+n} w_{m}^{*}=\frac{1}{M} e^{-i n \delta} .
\end{gathered}
$$

By observing the pattern in Eq.(36) for the particular case of $M=4$, the general expression for the average beamformer output, in the case of Toeplitz CSD matrix, can be derived:

$$
\begin{aligned}
\mathbf{w}^{\mathrm{H}} \mathbf{C} \mathbf{w}=c_{0} & +c_{1} \sum_{m=1}^{M-1} w_{m+1} w_{m}^{*}+c_{1}^{*} \sum_{m=1}^{M-1} w_{m} w_{m+1}^{*} \\
& +c_{2} \sum_{m=1}^{M-2} w_{m+2} w_{m}^{*}+c_{2}^{*} \sum_{m=1}^{M-2} w_{m} w_{m+2}^{*} \\
& +c_{M-1} w_{M} w_{1}^{*}+c_{M-1}^{*} w_{1} w_{M}^{*} \\
=c_{0} & +\frac{M-1}{M} e^{-i \delta} c_{1}+\frac{M-1}{M} e^{i \delta} c_{1}^{*} \\
& +\frac{M-2}{M} e^{-i 2 \delta} c_{2}+\frac{M-2}{M} e^{i 2 \delta} c_{2}^{*} \\
& +\frac{1}{M} e^{-i(M-1) \delta} c_{M-1}+\frac{1}{M} e^{-i(M-1) \delta} c_{M-1}^{*}
\end{aligned}
$$

The pattern of the coefficients in Eq.(39) shows that they implement a shading of the coherence function by a "lifted" triangular window, which has a value of one at the central point of the function (element $c_{0}$, corresponding to zero spacing), and 
decreases linearly with increasing magnitude of the spacing, both towards negative and towards positive values of the spacing, reaching the value $1 / M$ at the maximum spacing magnitude.

The triangular coherence-function shading described above is intrinsic to the CBF operation, and combines with any other array shading that can be introduced in the processing. The remainder of this study shows that, even when no array shading is implemented, the triangular shading of the coherence function is not desirable for bottom-loss estimation, and describes a technique that is immune from this issue. More specifically, by using the results in Eq.(28) and Eq.(32), this study introduces an algorithm for fast, improved-resolution BL estimation from array data, which also removes the limitations imposed by the triangular shading implicit in Eq.(13).

In this algorithm, first $\mathbf{C}_{\omega}$ is estimated by averaging array data over an adequate number of snapshots [see Eq.(8)]; if the field is only due to surface ambient noise, the CSD matrix is (approximately) Toeplitz and an average along the diagonals provides an estimate $\hat{C}_{\omega}(z)$ of $C_{\omega}(z)$. Furthermore, the estimated $\hat{C}_{\omega}(z)$ can be extended to the negative side of $z$ according to Eq.(32), windowed as desired, and its DFT taken between $\hat{C}_{\omega}(-L)$ and $\hat{C}_{\omega}(L)$, i.e. over $(2 M-1)$ samples. The ratio of the portions of the DFT of $\hat{C}_{\omega}(z)$ on the positive and negative sides of the $k$ axis (the discrete equivalent of Eq.(28)) provides an estimate of the power reflection coefficient $R(k)$. No further correction is needed if the array is sufficiently close to the bottom, and $R(k)$ can then be mapped back to angle space $\theta_{r}$ and used to estimate the BL according to Eq.(11). Sections 6.3 and 6.4 show how this technique can increase the 
angular resolution of the estimated bottom loss both in simulated and experimental shallow-water scenarios.

\subsubsection{Examples in simple scenarios}

The result in Eq.(32) deserves some more attention, as it may appear counterintuitive initially: For example, it does not hold for the case of a single point source. If such source were at depth $h_{s}$ and range $r$ from a receiver at depth $h$ (Figure 1 can still be used as reference), the normal-mode expression for the resulting pressure field at the receivers at a given frequency $\omega$ would be: 83

$$
\begin{aligned}
p_{\omega}(r, h) & \approx A\left(h_{s}\right) \sum_{m=1}^{\infty} \psi_{m}\left(h_{s}\right) \psi_{m}(h) i H_{0}^{(1)}\left(\kappa_{m} r\right), \\
A\left(h_{s}\right) & =\frac{1}{4 \rho\left(h_{s}\right)},
\end{aligned}
$$

Where $\rho$ is the water density, $\psi_{m}$ is the mode shape function, $\kappa_{m}$ is the modal wavenumber, and $H_{0}^{(1)}\left(\kappa_{m} r\right)$ is the Hankel function of the first kind [note that, in the interest of readability, in this section the notation is slightly different from that in Section 3.2; letting $\mathbf{r}=(r, h)$, the equivalence between the two notations is given by $\left.p_{\omega}(r, h)=p_{r}(\omega)\right]$

Using three receivers positioned along a vertical line at depths $(D-d), D$, and $(D+d)$, the resulting coherence functions between the center receiver and the other two would be: 


$$
\begin{aligned}
C_{\omega}(d) & =p_{\omega}(r, D) p_{\omega}^{*}(r, D+d) \\
& \approx A^{2}\left(h_{s}\right) \sum_{m=1}^{\infty} \sum_{n=1}^{\infty} \psi_{m}\left(h_{s}\right) \psi_{m}(D+d) \psi_{n}\left(h_{s}\right) \psi_{n}(D)\left[i H_{0}^{(1)}\left(\kappa_{m} r\right)\right]^{*} H_{0}^{(1)}\left(\kappa_{n} r\right) ; \\
C_{\omega}(-d) & =p_{\omega}(r, D) p_{\omega}^{*}(r, D-d) \\
& \approx A^{2}\left(h_{s}\right) \sum_{m=1}^{\infty} \sum_{n=1}^{\infty} \psi_{m}\left(h_{s}\right) \psi_{m}(D-d) \psi_{n}\left(h_{s}\right) \psi_{n}(D)\left[i H_{0}^{(1)}\left(\kappa_{m} r\right)\right]^{*} H_{0}^{(1)}\left(\kappa_{n} r\right) .
\end{aligned}
$$

Due to the difference in the depth dependence of the mode shape functions $\psi_{m}$, Eq.(41) does not necessarily imply that Eq.(32) would hold in this case. However, the surface noise considered in this work is different from a single point source, and its peculiar nature gives rise to the result in Eq.(32). For instance, in the case of surface

noise in an isospeed deep ocean, Cron and Sherman's model ${ }^{64,65}$ expresses the spatial coherence function as: ${ }^{75}$

$$
C_{\omega}(z)=2\left[\frac{\sin (2 \pi z / \lambda)}{2 \pi z / \lambda}+\frac{\cos (2 \pi z / \lambda)-1}{(2 \pi z / \lambda)^{2}}\right]-2 i\left[\frac{\cos (2 \pi z / \lambda)}{2 \pi z / \lambda}-\frac{\sin (2 \pi z / \lambda)}{(2 \pi z / \lambda)^{2}}\right]
$$

which yields the result in Eq.(32) exactly.

\subsection{Application to data}

\subsubsection{Application to simulated data}

Section 6.4 presents the results of applying the technique proposed in this study to measured data. In order to facilitate the interpretation of those results, this section applies both this technique and Harrison and Simons' technique to CSD matrices obtained from an OASN simulation. Since the simulated environment is perfectly known, in this case ground-truth bottom loss can be obtained from the power 
reflection coefficient computed by a theoretical model, ${ }^{2}$ and used to judge the quality of the results.

The geoacoustic parameters for this test are shown in TABLE II. Two array configurations are used, with 16 and 32 hydrophones; the inter-sensor spacing is $0.18 \mathrm{~m}$ in both cases, with the shallowest hydrophone at a depth of $180 \mathrm{~m}$, i.e. at $20 \mathrm{~m}$ from the bottom, to minimize the effect of attenuation. In the remainder of this dissertation, the proposed technique will be referred to as "high resolution bottomloss estimation" (HR-BL). Figure 9 shows the bottom-loss theoretical prediction, the HR-BL, and the CBF estimate at $2500 \mathrm{~Hz}$, from OASN-generated CSD matrices of sizes $32 \times 32$ and $16 \times 16$. The spatial coherence function for HR-BL is estimated by averaging the elements of the CSD matrix along the diagonals parallel to the main diagonal (exploiting the Toeplitz character of the matrix); the function is then "doubled" by extension to the negative $z$ values and tapered with a Tukey window with 0.6 taper width (the same used to shade the array when computing the CBF estimate). The DFT of this extended coherence function is computed as a Fast Fourier Transform and the reflection coefficient is estimated as the ratio of the halves of the resulting $k$ spectrum, as indicated in Eq.(28).

For both array lengths, the HR-BL results follow more closely the theoretical prediction, particularly in the 32-element case (Figure 9-a). Moving from 32 to 16 elements (Figure 9-b), the CBF experiences a significant loss of resolution, failing to recover most of the details of the peaks. On the other hand, the result of the 16element HR-BL processor is very close to the longer CBF over almost the entire 
angular range (except between the peaks). Results in Section 6.4 confirm that a 16element HR-BL processor can perform at a level comparable to a 32-element physical array when applied to measured data.

TABLE II. Water-column and bottom properties for the simulated cases; $\Delta$ is the layer thickness, $\rho$ is the density, $\alpha_{c}$ is the compressional volume attenuation, and $\lambda$ is the wavelength. Shear sound speed and attenuation were set to zero. Attenuation in water is set by OASN to its lower bound.

\begin{tabular}{ccccc}
\hline & $\Delta(\mathrm{m})$ & $c_{p}(\mathrm{~m} / \mathrm{s})$ & $\rho\left(\mathrm{kg} / \mathrm{m}^{3}\right)$ & $\alpha_{c}(\mathrm{~dB} / \lambda)$ \\
\hline Water & 200 & 1500 & 1000 & - \\
Sediment & 0.75 & 1550 & 1500 & 0.2 \\
Halfspace & $\infty$ & 1600 & 2000 & 0.15 \\
\hline \hline
\end{tabular}



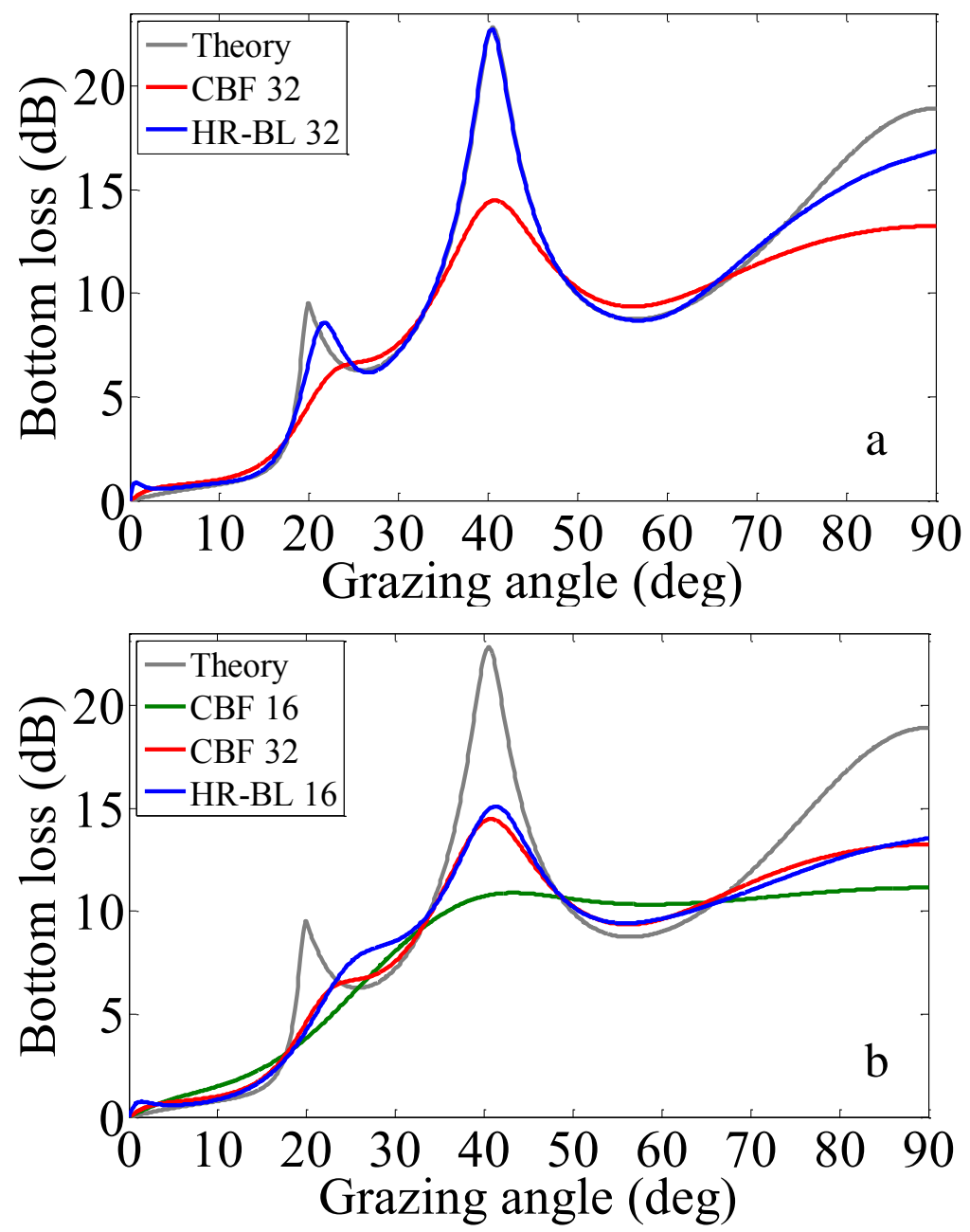

Figure 9. Estimated bottom loss at $2500 \mathrm{~Hz}$ from OASN data for the environment in TABLE I. (a): Theoretical bottom loss ("Theory"), HR-BL processor and CBF using $32 \times 32$ CSD matrices produced by OASN. (b): Same as in (a), but using $16 \times 16$ matrices (the CBF 32 curve is repeated to facilitate a direct comparison). In both cases, when using the same number of physical sensors the HR-BL curve is closer to the theoretical prediction than the CBF curve over almost the entire angular range. Note the significant degradation of the CBF when moving from 32 to 16 elements, and how the HR-BL 16 curve is very close to the CBF 32 curve.

To further highlight the benefits of HR-BL processing over CBF, Figure 10 shows the bottom loss estimated over the frequency range $25-4166 \mathrm{~Hz}$ for the same OASN data used in Figure 9, this time using a Hanning taper (the Tukey taper used in Figure 9 
can be too "aggressive" at low frequencies), and the pixel-by-pixel error between the values predicted by the theoretical model and the estimated ones. Although the Hanning taper does not maximize the performance of either technique in the upper part of the frequency range, the HR-BL processor is closer to the theoretical prediction along the ridge peaks and performs particularly well at the lower frequencies.
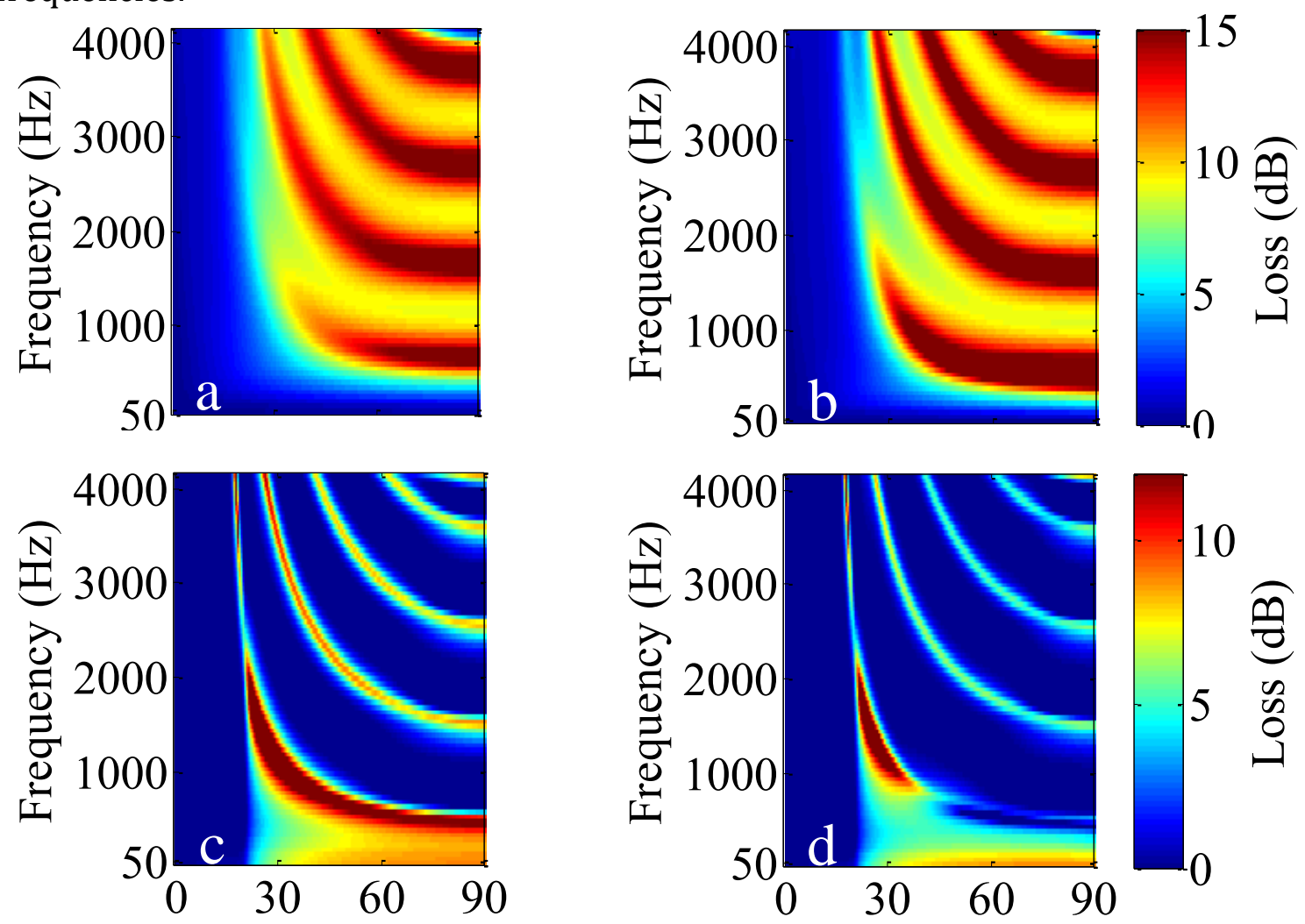

Grazing angle (deg)

Grazing angle (deg)

Figure 10. Estimated bottom loss and error over the frequency range $25-4166 \mathrm{~Hz}$ from OASN data for the environment in TABLE I. The bottom loss is estimated from the same $32 \times 32$ CSD matrix using a Hanning taper and CBF (a) and HR-BL processing (b). The error is computed as the pixel-by-pixel difference in $\mathrm{dB}$ between the bottom loss predicted using the model by Jensen et al. and that estimated by the CBF (c) and the HR-BL processor (d). 


\subsubsection{More on the Toeplitz character of the CSD matrix}

HR-BL processing is based on the assumption that the CSD matrix of the original array is Toeplitz, which is a known property for a surface-noise-only field. However, measured CSD matrices do not always exhibit a Toeplitz structure. As an example, Figure 11 shows the real and imaginary part of two CSD matrices obtained from the BOUNDARY-03 experiment ${ }^{48}$ by averaging 5 -minute segments collected about 40 minutes apart. Since the interest here is in the geometric structure of the matrices, rather than the values of their elements, to ease the comparison each matrix has been normalized so that the maximum absolute value of its elements is 1 . Two important differences are apparent. First, the CSD matrix in Figure 11-a,b does not show as clear a Toeplitz structure as the matrix in Figure 11-c,d. Second, its diagonal bands are wider, and do not decay as markedly when moving away from the main diagonal. These two differences appear to have a strong influence on the bottom loss estimated from these CSD matrices. The results shown in Figure 12 were obtained by applying conventional beamforming and HR-BL processing to the $32 \times 32$ matrices shown in Figure 11. In Figure 12-a, both curves drop below zero at low grazing angles, an implausible result for a field generated only by surface noise and therefore an indication that some fundamental assumption in the model is violated. In this case, the HR-BL curve shows very large oscillations, which are inconsistent with the curves in Figure 12-b (from data collected about 40 minutes later), which shows more plausible curves: The physical-array curve appears to be a smoothed version of the HR-BL curve and the latter shows more marked oscillations and a higher bottom loss 
around endfire. In other words, the HR-BL processor results compare to the CBF results in a manner similar to what was observed for the OASN simulation (see Figure 9).
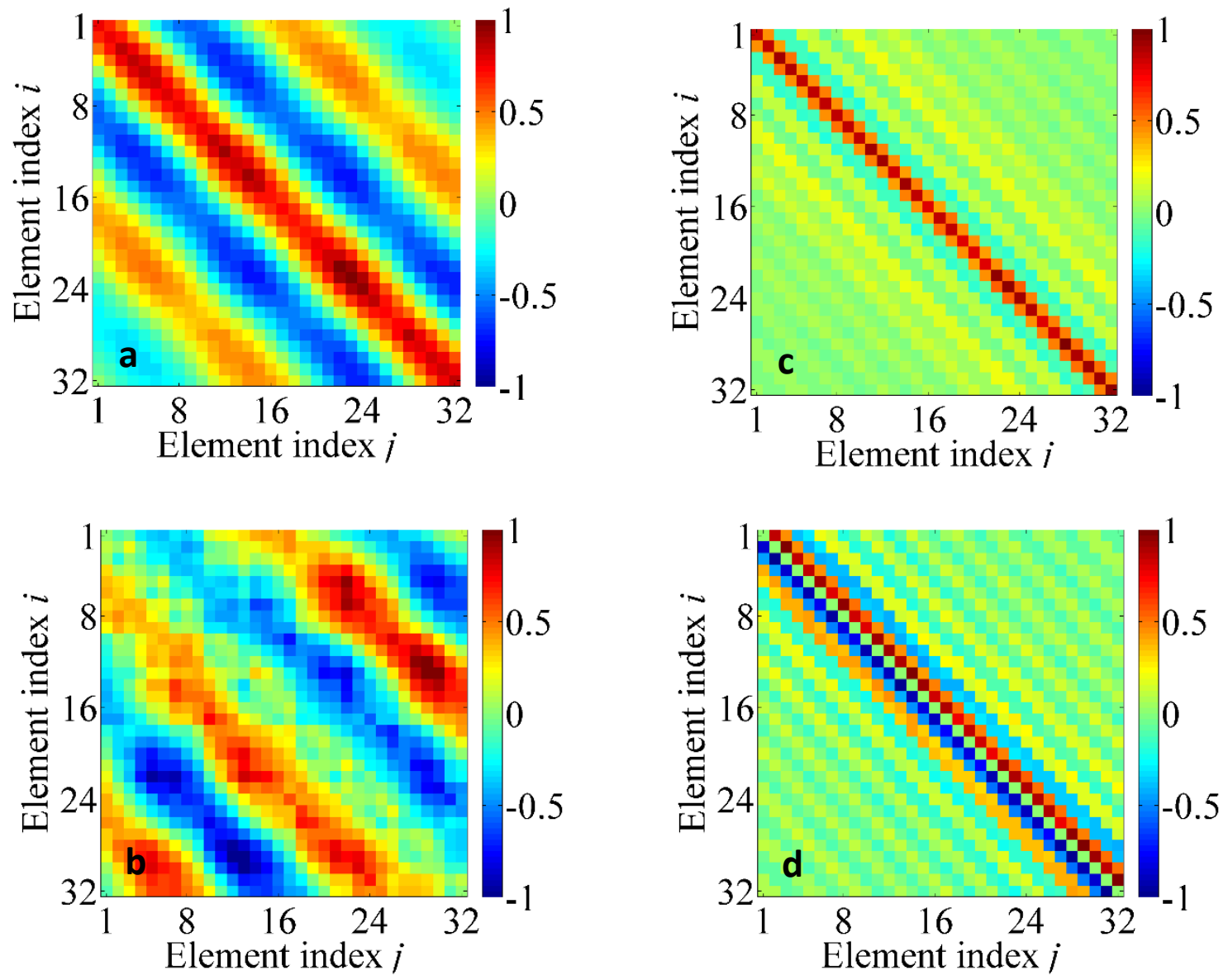

Figure 11. Real (top) and imaginary (bottom) parts of the normalized CSD matrices at $2156 \mathrm{~Hz}$, computed from two 5 -minute snapshots (collected about 40 minutes apart) from the BOUNDARY-03 experiment. The matrices in panels c-d appear to be closer to Toeplitz than the matrices in panels a-b. 

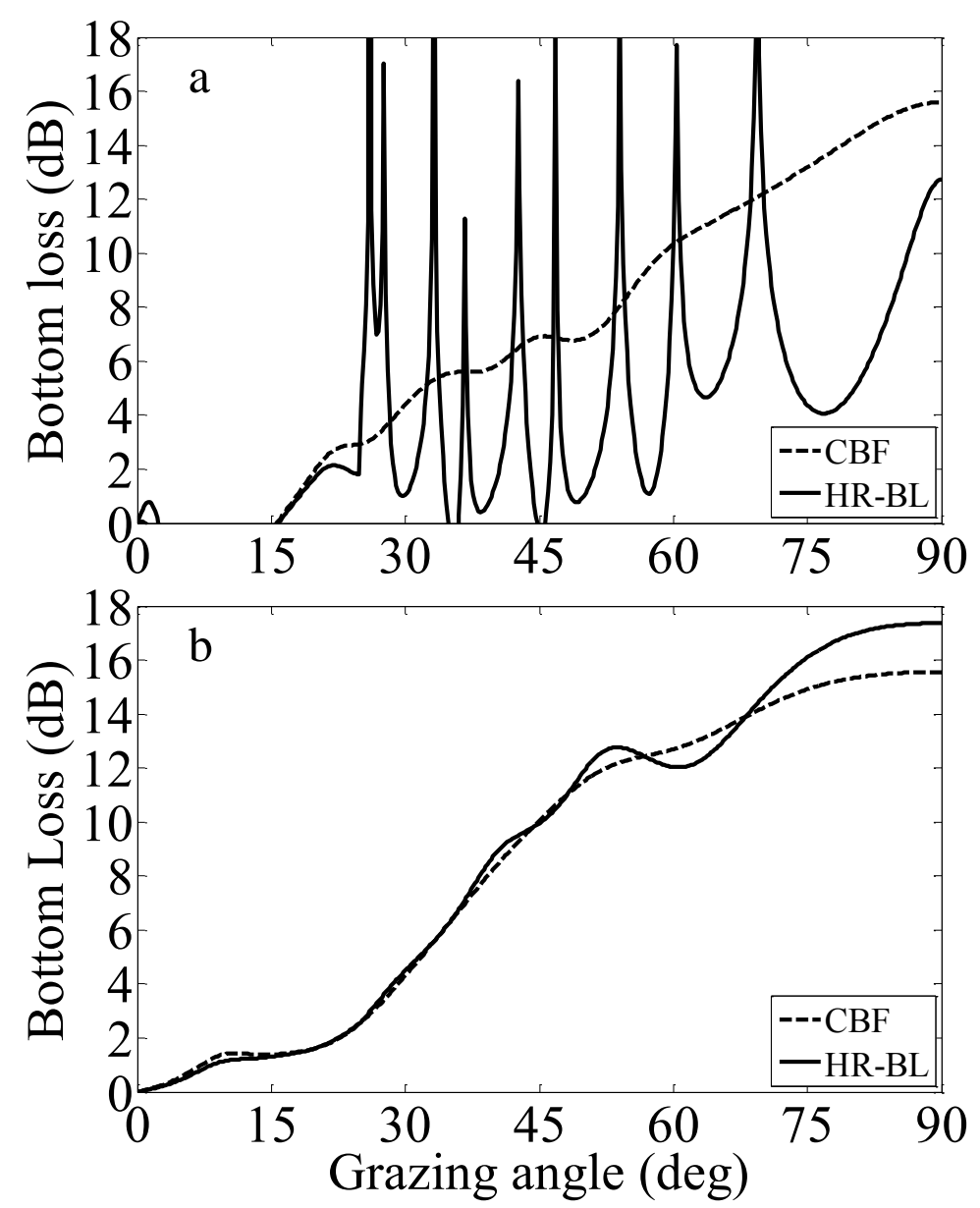

Figure 12. Bottom-loss curves: 32 -element CBF vs. HR-BL for the same data as in Figure 11. The drop below zero of the bottom-loss curves visible below $20^{\circ}$ in (a) (corresponding to Figure 11-a,b) is an indication that some violation of the model assumptions is occurring in this 5-minute average. The large oscillations of the HRBL curve are also inconsistent with the curve from data collected about 40 minutes later (b) (corresponding to Figure 11-c,d), where the two techniques compare in a manner analogous to what observed for the OASN simulation (see Figure 9).

The comparison between the two CSD matrices in Figure 11 raises the question of what is inducing such dramatic alterations in the structure of the matrices. One possible cause of the non-Toeplitz character of the matrix is array deformation. Harrison's model for the spatial coherence function contains a term that is a function of the elevation angle of the line joining the two receivers. When the array is assumed 
to be perfectly vertical (as in this study), this term equals one and therefore does not appear in Eq.(16). But if the array is deformed, in general the elevation angle of the line joining an arbitrary pair of sensors will vary depending on the particular pair chosen, changing the value of the additional term. This variation will reflect on the CSD matrix by introducing some variability along the diagonals. Given the arbitrary character of array deformation, it is hard to provide a systematic study of the influence of this effect on the CSD matrices. OASN simulations conducted with rather severe deformations showed increased variability along the diagonals, but not to the extent visible in Figure 11, failing in particular to produce the alteration of the band structure.

It is reasonable to think that a nearby discrete source such as a ship could be responsible for the effects observed in Figure 11 and Figure 12; Figure 13 supports this hypothesis by plotting the beamformer output at $2156 \mathrm{~Hz}$, as a function of steering angle and time. A loud interferer appears close to broadside around time 19:25, and approaches the array reaching the closest point around time 19:40, as indicated by the broadening of the angle covered by the interferer. The CSD matrix shown in Figure 11a,b corresponds to a 5-minute time average centered around time 19:27:30 (when the interferer's presence is quite strongly affecting the array output), while the matrix in Figure 11c,d is based on a time average centered around time 20:07:30 (when the interferer's influence is much reduced). 


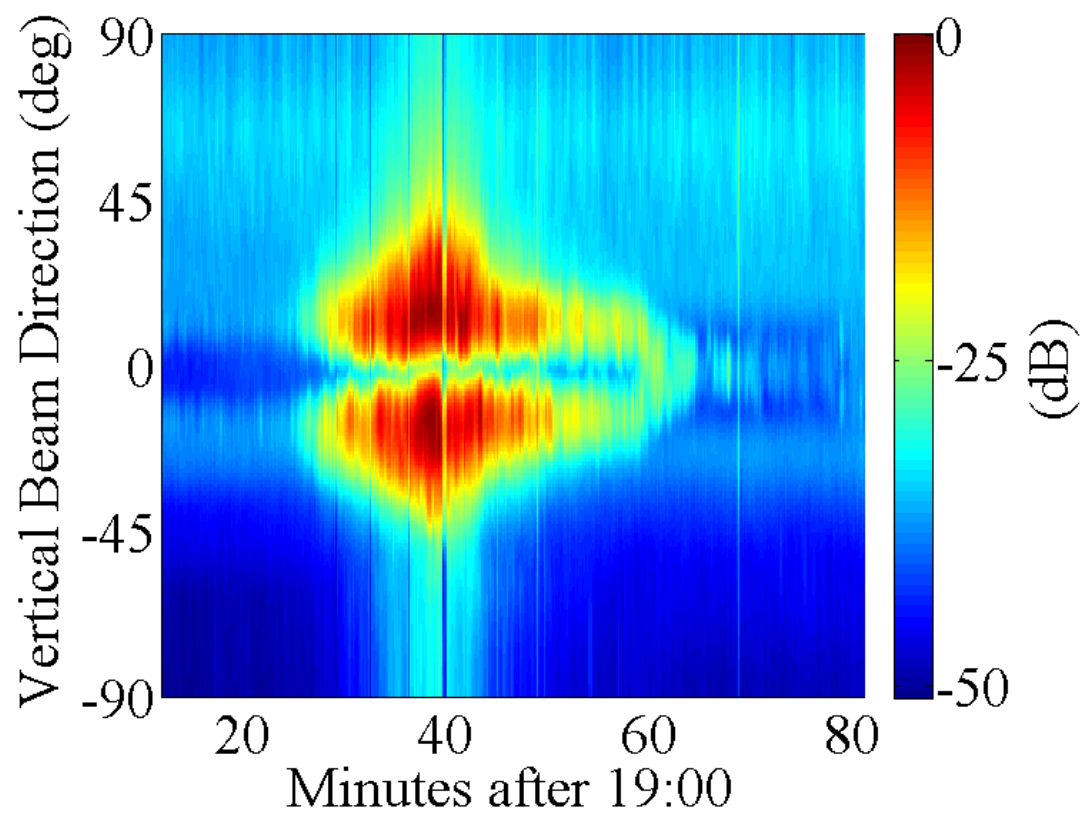

Figure 13. Beamformer output at $2156 \mathrm{~Hz}$, as a function of steering angle and time, from the dataset used to produce the CSD matrices in Figure 11. A loud interferer appears close to broadside around time 19:25, and approaches the array reaching the closest point around time 19:40, as indicated by the broadening of the angle covered by the interferer. The CSD matrix shown in Figure 11-a,b corresponds to a 5-minute time average centered around time 19:27:30 (when the interferer is quite strongly affecting the array output), while the matrix in Figure 11-c,d is based on an average centered around time 20:07:30 (when the interferer's influence is much reduced).

\subsection{Results}

In this section, the procedures described in Section 8.4 for simulated data are applied to the CSD matrices obtained from data collected at sea by 32-element arrays during three separate experiments by the NATO-STO Centre for Maritime Research and Experimentation (CMRE, La Spezia, Italy - formerly NATO Undersea Research Centre). The data represent measurements from two different vertical arrays, at six different locations. The dataset identifiers used in this chapter are reported in TABLE III, together with the basic features of the array and acquisition system. 
TABLE III. Datasets and array basic features.

\begin{tabular}{cccccc}
\hline Dataset ID & $\begin{array}{c}\text { Num. of } \\
\text { elements }\end{array}$ & Spacing (m) & $\begin{array}{c}\text { Sampling freq. } \\
(\mathrm{Hz})\end{array}$ & $\begin{array}{c}\text { Design freq. } \\
(\mathrm{Hz}) @\left(\begin{array}{c}\text { Deploy } \\
\mathrm{c}=1500 \mathrm{~m} / \mathrm{s}\end{array}\right.\end{array}$ & $\begin{array}{c}\text { ment } \\
\text { type }\end{array}$ \\
\hline VLA-03 & 32 & 0.50 & 6000 & 1500 & Drifting \\
MFA-03 & 32 & 0.18 & 12000 & 4166 & Drifting \\
MFA-04 & 32 & 0.18 & 12000 & 4166 & Drifting \\
GLASS12 & 5 & 0.1 & 90000 & 7500 & Moored \\
GLASS13 & 5 & 0.1 & 90000 & 7500 & Moored \\
SLIVA-14 & 32 & 0.18 & 50000 & 4166 & Moored \\
\hline \hline
\end{tabular}

For the location of these measurements, the only ground truth available is in the form of normal incidence measurements (e.g., seismic chirp sonar), which can provide information about the layering of the bottom, but not the bottom loss, which is of interest in this study. For this reason, Figure 14, Figure 15, and Figure 16 show two CBF lines, corresponding to bottom-loss estimates obtained using the full array (32 elements), and a sub-array composed of the first 16 elements. The third line, in each of the plots, is the bottom loss estimated by the HR-BL processor using data from the same sensors as the 16-element CBF. Since no bottom-loss ground truth is available, the estimate from the longer array is assumed to be the better one, and the performance of the 16-element HR-BL processor can be assessed by comparison with that of the two CBF results. All the CSD matrices were obtained by averaging 5 minutes of data. Both the HR-BL coherence function before the DFT, and the array 
data used by the CBF are tapered using a Taylor window with $-30 \mathrm{~dB}$ maximum sidelobe level (compared to the main lobe).

For the 16-element cases, the CBF curves show a marked degradation in angular resolution, in the form of less pronounced, wider peaks and valleys, and a generally lower loss estimated towards $90^{\circ}$. The HR-BL curves are obtained by processing only the first 16 elements of the array. The HR-BL curves appear largely immune to the degradation experienced by the 16-element CBF, very closely resembling the performance of the 32-element CBF. Note that, given the larger inter-element spacing, the frequencies in the VLA-03 case are lower than in the others, but CBF and HR-BL compare in similar terms. 

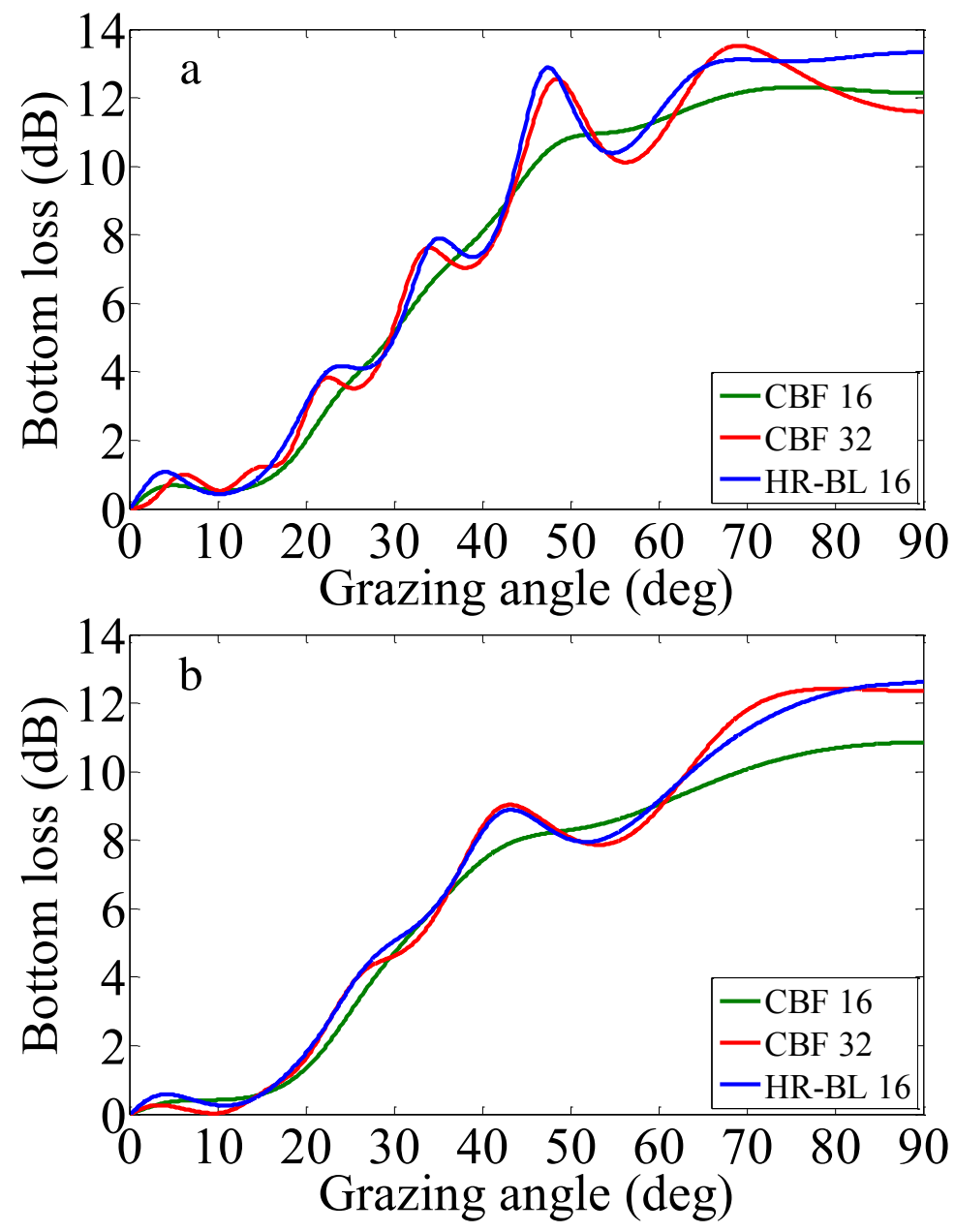

Figure 14. Bottom-loss curves computed from two 5-minute averages (data from the VLA-03 dataset) at $1313 \mathrm{~Hz}$ (a) and $972 \mathrm{~Hz}$ (b): Conventional beamforming (CBF) for 32-element and 16-element physical array vs. 16-element HR-BL processor using a Taylor taper with $-30 \mathrm{~dB}$ sidelobe level. In both cases the 16-element HR-BL processor reproduces the features of the 32-element CBF curve more faithfully than the 16-element CBF, and limits the bottom-loss disruption around endfire. 

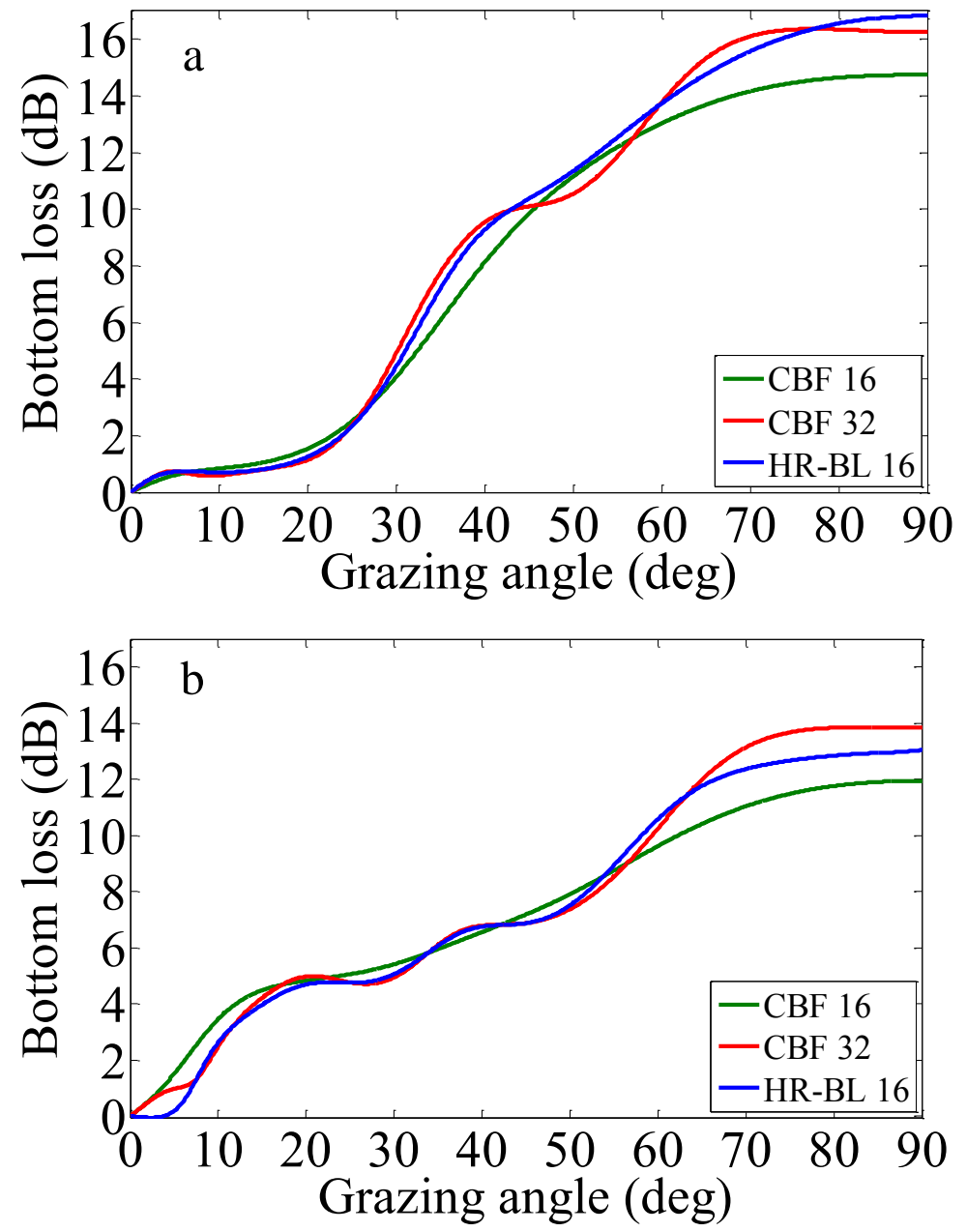

Figure 15. Bottom loss curves computed from two 5-minute averages (data from the MFA-03 dataset) at $2000 \mathrm{~Hz}$ (a) and $2250 \mathrm{~Hz}$ (b); processing and naming conventions are the same as in Figure 14. 

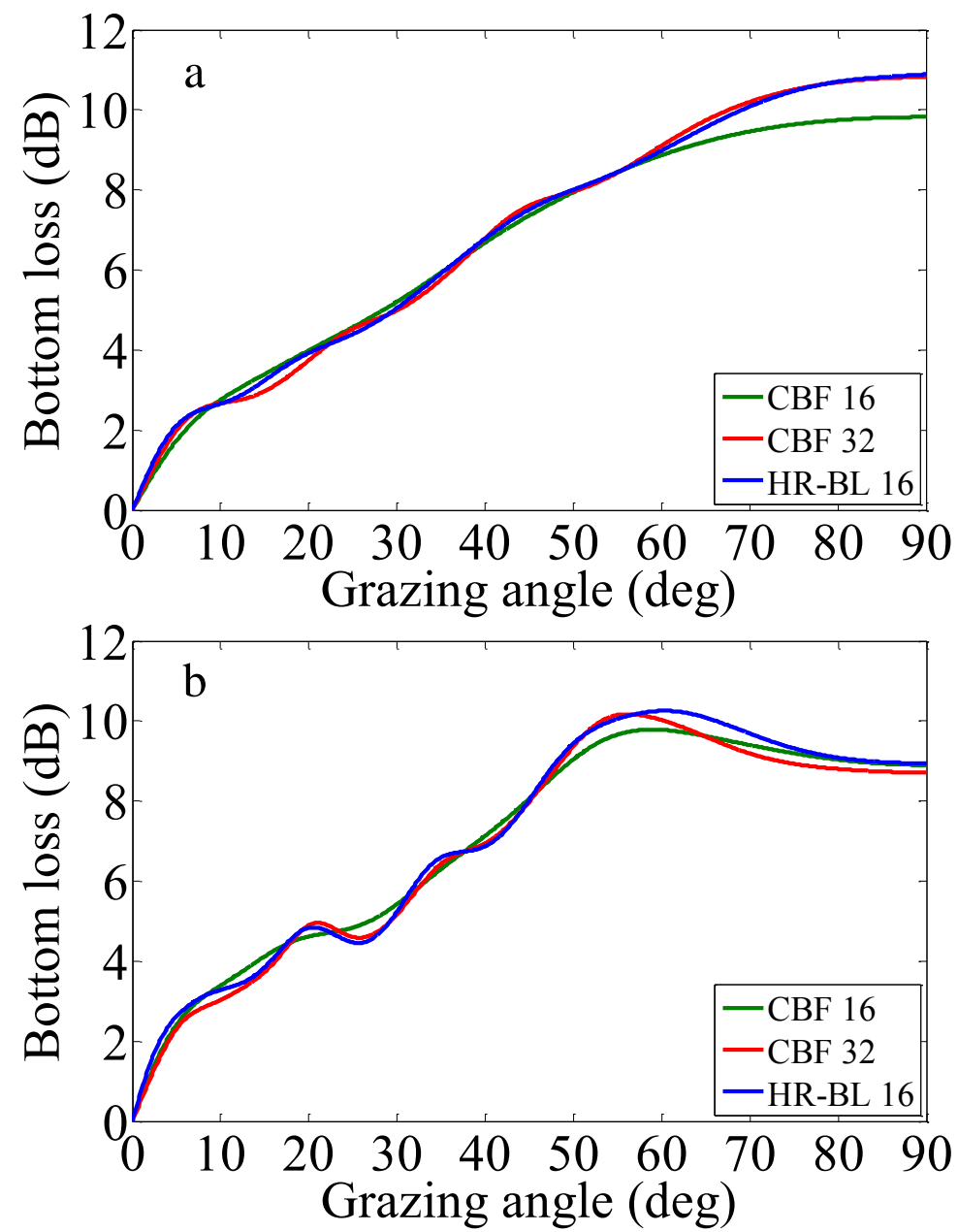

Figure 16. Bottom loss curves computed from two 5-minute averages (data from the MFA-04 dataset) at $2414 \mathrm{~Hz}$ (a) and $3070 \mathrm{~Hz}$ (b); processing and naming conventions are the same as in Figure 14. 


\subsection{Summary}

In this section, a previously introduced derivation in frequency-wavenumber domain of the bottom plane-wave power reflection coefficient from the array coherence function has been extended to include the effects of volume attenuation and variable sound speed in the water column. The main result is that, under certain conditions, for a surface-noise-only field it is possible to obtain the reflection coefficient (and therefore the bottom loss) by computing the Fourier transform of the coherence function $C_{\omega}(z)$. A technique has been presented, and theoretically justified, that improves on the bottom-loss estimate provided by the matrix-product implementation of conventional beamforming, by exploiting the Toeplitz structure of the noise-only CSD matrix and a DFT implementation of beamforming. The technique has been demonstrated both on simulated and measured data. When the estimated cross-spectral-density matrix obtained from array data is sufficiently close to Toeplitz, experimental results show that a 16-element array can improve the estimated bottom loss, achieving an angular resolution comparable to that of a matrix-product implementation of $\mathrm{CBF}$ on a 32-element array. 


\section{Application to arrays suitable for AUV deployment}

Harrison and Simons' technique has proved to perform consistently and reliably in the past, but those studies used arrays of lengths of at least $5.5 \mathrm{~m}$, and exclusively adopted conventional beamforming for obtaining the estimated spatial power spectrum. The recent introduction of AUVs as operational platforms has raised the question of whether they could be employed as bottom-survey tools using this technique. Particularly, Arvelo ${ }^{27}$ has proposed inserting very short arrays in the nose of AUVs, and the NATO-STO CMRE has developed a short array that can be mounted on the nose of an AUV. ${ }^{78}$ In these cases, the array length can range between a few tens of a meter and less than $2 \mathrm{~m}$, and these lengths will be considered as viable for AUV deployment in the remainder of this dissertation.

Since the angular resolution of the estimated bottom loss deteriorates as the array length reduces [see Eq.(15) and related discussion], it is natural to wonder how the performance of this technique is impacted when moving to array lengths suited for AUV deployment. In the case of this particular application, the literature does not provide an answer: Arvelo's work, which was limited to simulated data, remains the only study available. He proposed overcoming the resolution problem by aggressive adaptive beamforming, but showed significant results only at high frequencies, and below the critical angle. Part of the research described in this study aims at filling this gap by investigating the performance of short arrays using both Harrison and Simons'

original technique, and the new algorithms proposed in this dissertation, both in simulation and on measured data. 


\subsection{Results from the GLASS experiment}

The GLASS experiment represents the first attempt at investigating AUV-based bottom survey through ambient-noise processing. The main agency behind the project is the Centre for Maritime Research and Experimentation (La Spezia, Italy), part of the North Atlantic Treaty Organization, Science and Technology Organization (NATO-STO), and the author has collaborated with them taking part in the GLASS 2013 and REP14-MED campaigns both on the field, and by subsequently processing the data collected. 

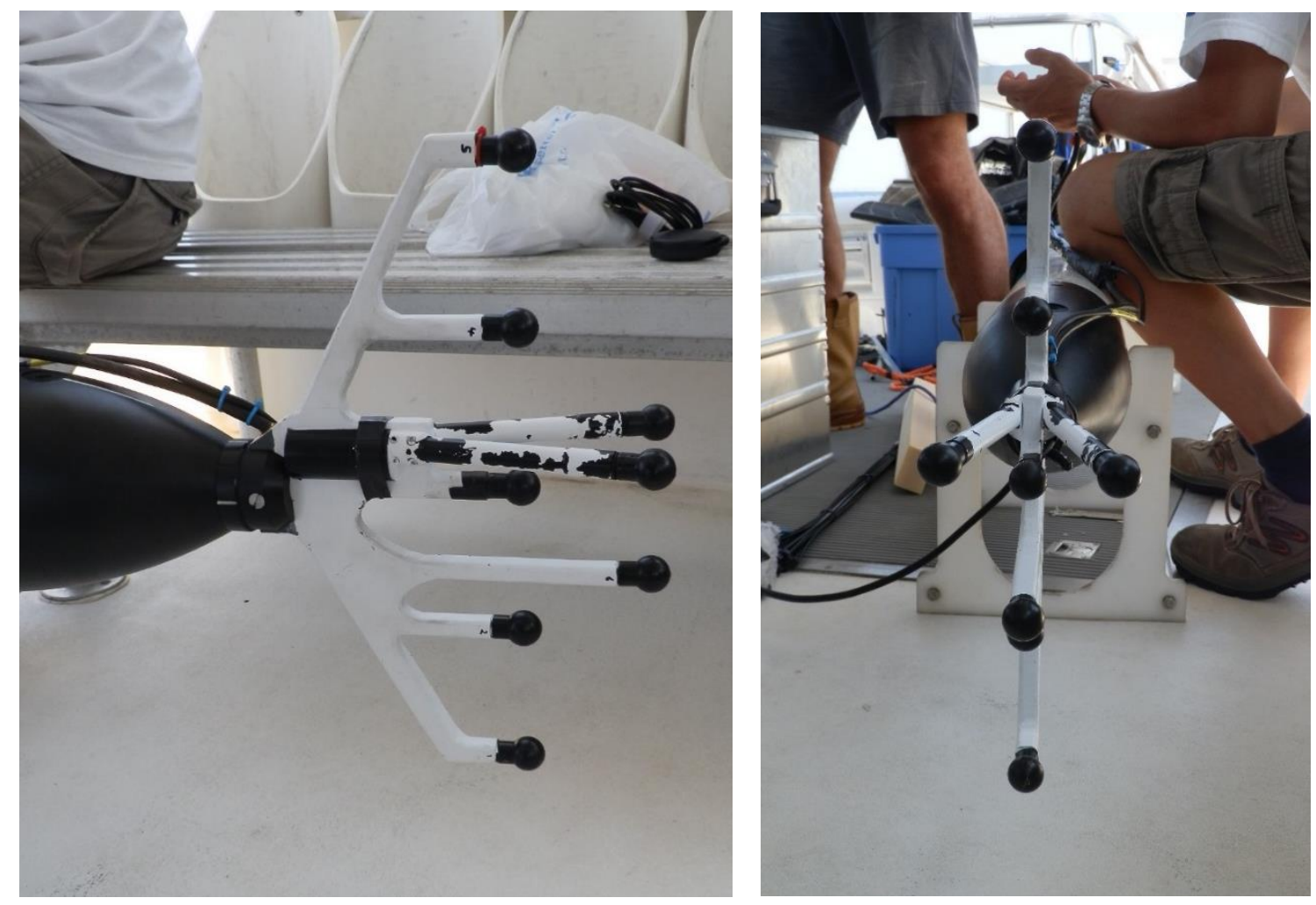

Figure 17. The GLASS volumetric array — Side and front view.

The goal of the project is to transition Harrison and Simons' technique, so far implemented only using moored or drifting arrays, to an autonomous vehicle equipped with a compact array. A new type of hybrid AUV named eFOLAGA 61 is being jointly developed by the University of Genova (Genova, Italy), the CMRE and the manufacturer GraalTech s.r.l (Genova, Italy). The vehicle is equipped with jet pumps and blade propellers, which has the advantage, over ordinary gliders, of more flexible maneuver features, including e.g. diving in the vertical direction, hovering at specific heading and depth and rotation on the spot. For GLASS, the CMRE developed a nosemounted combined vertical and tetrahedral array (see Figure 17 and Figure 18), 
comprising a total of 8 elements, 5 of which are on a straight (vertical) line with $0.1 \mathrm{~m}$ spacing. The central element of the line array is also one of the vertices of the tetrahedral array, which includes 3 more elements; the spacing between any two sensors in the tetrahedral array is still $0.1 \mathrm{~m}$.

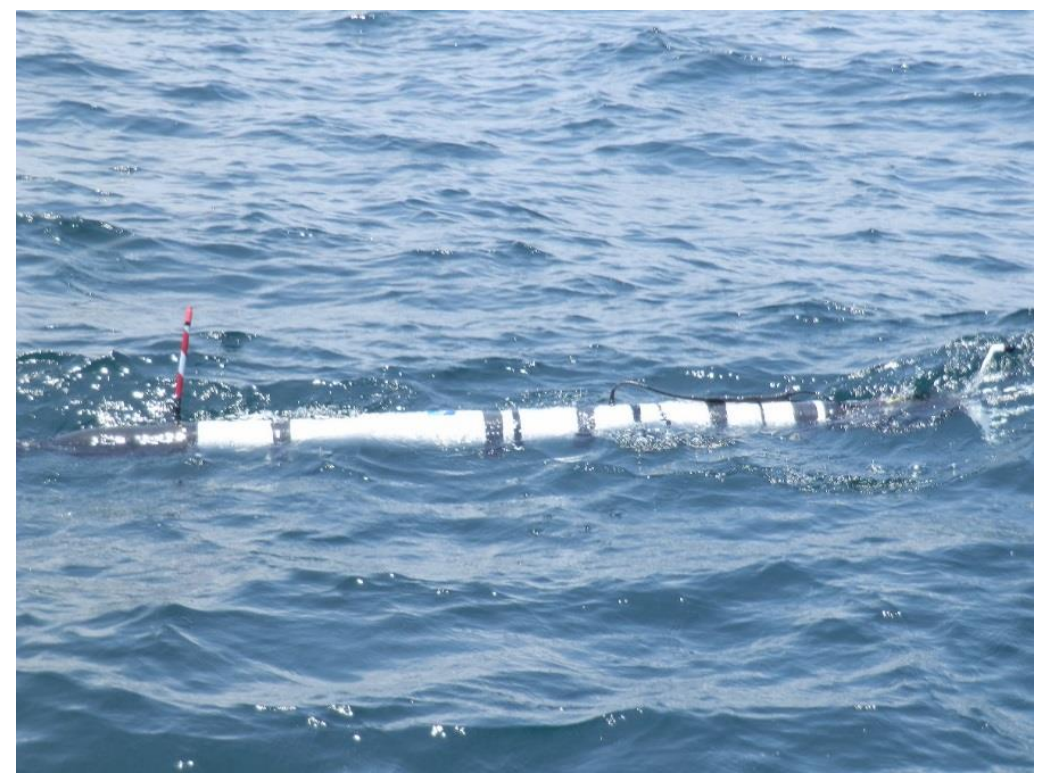

Figure 18. The eFolaga, with the GLASS volumetric array mounted on its nose, beginning one of its missions.

\subsubsection{GLASS sea trials}

The AUV with mounted data-acquisition package was deployed for the first time in the Summer of 2012 (GLASS'12) off the Versilian Coast, Mediterranean Sea (Italy), ${ }^{78}$ with the engineering objective of integrating the nose-mounted and combined vertical and tetrahedral array with the remaining electronic control and sensing devices on the eFOLAGA. Some ambient-noise data were collected during this trial, with the AUV mounted on a frame fixed on the bottom. 
In the Summer of 2013, the author took part in the GLASS '13 experiment, off the coast of Panama City, Florida, US, collaborating to the phases of mission planning, equipment deployment and retrieval, data collection and data processing. The scientific goal was to provide experimental validation of the performance of an AUVmounted short array in ambient-noise-based bottom characterization. This would be accomplished by collecting data to estimate bottom loss and layering structure from sea surface ambient noise and distant shipping in an environment different from the GLASS12 region, and by comparing ambient noise derived seabed properties with independent measurements obtained during the ONR sponsored Target and Reverberation Experiment 2013 (TREX13), conducted during April-May 2013 in the same area. A total of 80 minutes of ambient noise data was identified as usable for seabed characterization during the GLASS'13, where 70 minutes of the data were acquired while the AUV was bottom moored (moorings over two days), and 10 minutes of useful data while the vehicle was kept at constant depth and gliding through the water. The study described here represents the first investigation of the dataset.

\subsubsection{Array performance characterization: Sensitivity to tilt and bottom properties}

While the moored/drifting arrays used in the past to implement Harrison and Simons' technique were several meters long, the 5-element linear array mounted on the eFolaga AUV has a total length of $0.4 \mathrm{~m}$. This fact alone indicates that a significant deterioration in angular resolution of the bottom-loss estimate must be expected, and 
in fact bottom-loss plots produced by GLASS data look markedly different from the ones observed above, as a comparison between Figure 4 and Figure 19 demonstrates.
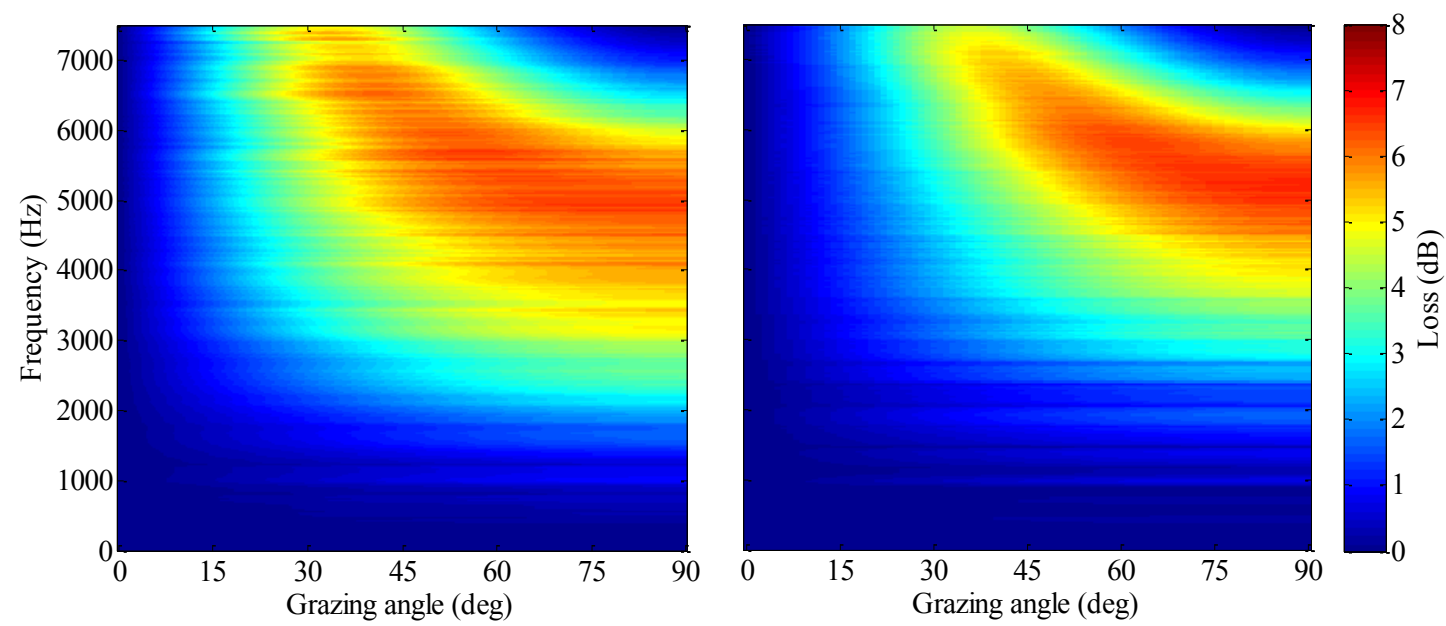

Figure 19. Estimated bottom loss from GLASS'12 (left) and GLASS'13 (right) data. In both cases, the limitations imposed on the estimate by the reduced array length are apparent, when comparing these plots with Figure 4.

After the sea trial in June 2013, the author spent 6 weeks processing/analyzing the GLASS data as a Visiting Researcher at the CMRE. Since no systematic study investigating the effects of such short arrays on the estimated bottom loss had been conducted before, the first part of the term at the CMRE was spent to gain a better understanding of these issues, to which this section is devoted. The details on what kind of quantitative and qualitative information can be extracted from bottom-loss maps such as the ones in Figure 19 will be given in the next section.

The differences in the plots of Figure 19 suggest that the seabed properties must be different in the two cases. Although results from previous experimental activities at the two sites confirm this circumstance, the appearance of the GLASS'13 plot raised some legitimate suspicion. The two salient features of the plot are its very high critical 
angle and very low estimated bottom loss below about $4500 \mathrm{~Hz}$. In previous work the author had observed that such a combination of features can present itself when the array is tilted, i.e. its longitudinal axis forms an angle smaller than $90^{\circ}$ with the horizontal. Since the amount of data collected is not large, rather than discarding these data altogether, it is important to try and establish whether the plot's appearance is due to adverse deployment conditions (tilted array) or represents a physically plausible result. In this case, during data collection the AUV was tethered with rope and ballast (contrary to the specially manufactured rigid frame used during GLASS'12), which may introduce current- and wave-induced tilt of the vertical line array and, therefore, possibly degrade the performance of the seabed characterization. The array was deployed twice in this configuration:

1) bottom moored with only the acquisitions system turned on, to evaluate the state of the array and self-noise of the acquisition system;

2) bottom moored with both acquisition system and control unit powered.

The latter would indicate any motion of the vehicle induced by current and waves that would alter the array orientation. However, due to a software bug the propeller of the vehicle turned on a couple of minutes after the latter deployment and contaminated the noise measurements and attitude of the vehicle. However, in the first couple of minutes after the deployment without the propeller turned on, the attitude sensors did indicate a roll of around $3^{\circ}$ and pitch close to $8^{\circ}$. The question now is to establish whether this would be enough to cause a significant alteration of the bottom-loss estimate. 
The question was addressed through numerical modeling by beamforming noiseonly CSD matrices produced by OASN in an environment chosen to emulate the experimental set-up during the GLASS'13 deployment, and introducing increasing amounts of tilt in the array orientation. The study was repeated for several different bottom types, varying in particular the sound speed (which directly influences the critical angle) and the bottom density (which influences the bottom-loss level above the critical angle). The results clearly indicated that a tilt of $10^{\circ}$ would not have a significant impact on the estimated bottom loss, but that some physically sensible combinations of seabed properties, coupled with the array's low angular resolution, can result in plots like the one in the right panel of Figure 19.

\subsubsection{GLASS line-array data processing for bottom-loss estimation}

This section presents an example of what kind of qualitative and quantitative information can be obtained from bottom-loss estimates produced by the GLASS array. In general, one can expect this task to be harder than in the case of longer arrays, and a comparison between Figure 4 and Figure 19 immediately confirms this circumstance. The two plots shown in Figure 19 differ from the ones produced by longer arrays in that they appear to be "noisier", and do not seem to show evidence of striations (which would indicate layering in the bottom).

Differences can also be observed between the two GLASS plots; as stated above, this is unlikely to be due to array tilt, and is believed to be due to the bottom physical properties, coupled with the low array resolution. Now, the seabed properties in part of the GLASS'12 experimental area can be characterized as clay or clay-silt based on 
previous experimental activities and by core data acquired during the experiment. The GLASS'13 site has been visited several times in the past by the majority of the Principal Investigators of the TREX, and results from these previous experiments indicate that the seabed is composed mainly of sand with shell debris and clay/silt inclusions. Therefore, the AUV has been deployed in two different shallow-water environments during the GLASS' 12 and GLASS' 13 experiments. The differences in the two plots show that the measured loss is affected by the different bottom types. This finds confirmation in the geoacoustic inversion study described below.

Another salient feature in the GLASS plots is the absence of striations, which would in theory indicate a bottom with no layering ("halfspace"); however, this feature could also be a consequence of the poor angular resolution of the array. In the case of GLASS'12, the seismic profiling at the site did not reveal any clear bottom layering structure, whereas this type of information is not available for GLASS'13. It is therefore hard from these plots to understand which of the circumstances is presenting itself in this case.

\subsubsection{Use of estimated bottom loss for geoacoustic inversion}

The second part of the analysis carried out at the CMRE was aimed at obtaining more quantitative results, by investigating the feasibility of inverting the data for geoacoustic parameters of the bottom. The derived reflection loss from the GLASS'12 and GLASS'13 sites has been used in a model-based inference method to estimate the geoacoustic properties of the bottom. In this analysis, the seabed is described as an infinite halfspace with only three unknown parameters, namely density, attenuation 
and sound speed. The inversion is based on a very simple algorithm, which does not aim at competing with state-of-the art systems: The aim of this study, rather than accurate determination of the seabed parameters, is a first assessment of the sensitivity of this array to different bottom types.

The main components of the search algorithm are the cost function and the search space. The algorithm compares the bottom loss estimated from data at all grazing angles and in the $1-7.5 \mathrm{kHz}$ frequency range to the entire corpus constituting the search space and produces as solution the bottom type that results in the lowest costfunction value. The cost function is the RMS of the pixel-by-pixel difference between the data-estimated bottom loss and the model-derived bottom loss for a given combination of the bottom parameters. The search space included bottom types given by all possible combinations of the discretized values of the three search parameters: 8 values for the attenuation covering the range from $0.2-1.6 d B / \lambda ; 15$ values for the sound speed in the range $1440-2000 \mathrm{~m} / \mathrm{s}$, and 41 density values in the range $1000-3000 \mathrm{~kg} / \mathrm{m}^{3}$, for a total of 4920 configurations constituting the search space.

The corpus of bottom-loss values (as a function of frequency and grazing angle) constituting the search space was obtained by running for each configuration the following procedure:

1) A model found in literature ${ }^{2}$ was implemented and run to compute the planewave power reflection coefficient for each combination of the bottom parameters. 
2) To compute the spatial coherence function between each pair of hydrophones, the implementation of Harrison's model ${ }^{73,74}$ introduced in Section 5 was used. This receives as input the reflection coefficient generated in step 1), together with the water-column physical parameters and positions of the array hydrophones.

3) The coherence function resulting from Harrison's model is used to build the CSD matrix, which is then beamformed.

4) The result is used to estimate the bottom loss.

Having implemented Jensen et al.'s model and Harrison's model was key to the performance of this procedure. The same study would have been possible by running OASN simulations for all the bottom types, but it is estimated that it would have required running times higher by one to two orders of magnitude. Excellent agreement between the implemented models and OASN has been verified by comparing the results over a number of test cases (which included bottom layering). It seems important to state that the $\mathrm{CBF}$ has in this case been applied using a Taylor taper. This taper is not as established in its use in signal processing as others (such as the Hanning taper), and one of its salient features is that its value at the end points is not zero. Although this can in some cases introduce undesirable artifacts in signal processing, the Taylor taper empirically appeared to provide better "contrast" in the bottom-loss plots for this type of array, and this feature is very desirable when working with a cost function as the one used in this study. Since the taper is applied both to the measured data and to the synthetic data it is compared to for the search, 
any "features" due to the taper would appear in both cases, and should therefore not have any deleterious impact on the search result.

Two examples of the results obtained by the exhaustive search algorithm for GLASS'12 and GLASS'13 data are shown in TABLE IV, Figure 20 and Figure 21.

TABLE IV. Results of GLASS data inversion for two data samples.

\begin{tabular}{cccc}
\hline Data file & $\begin{array}{c}\text { Optimal } \\
\alpha_{p}(\mathrm{~dB} / \lambda)\end{array}$ & $\begin{array}{c}\text { Optimal } \\
c(\mathrm{~m} / \mathrm{s})\end{array}$ & $\begin{array}{c}\text { Optimal } \\
\rho\left(\mathrm{kg} / \mathrm{m}^{3}\right)\end{array}$ \\
\hline glass_2012_07_24_09_28_10 & 1 & 1560 & 2250 \\
glass13_2013_06_13_17_43_08 & 0.6 & 1600 & 1750 \\
\hline \hline
\end{tabular}

The sensitivity of the search to the three parameters has been examined in a number of cases, showing consistently that the sound speed is the most sensitive parameter, i.e. the one that induces the largest variations of the cost function for a given configuration, followed by the density and the attenuation. 


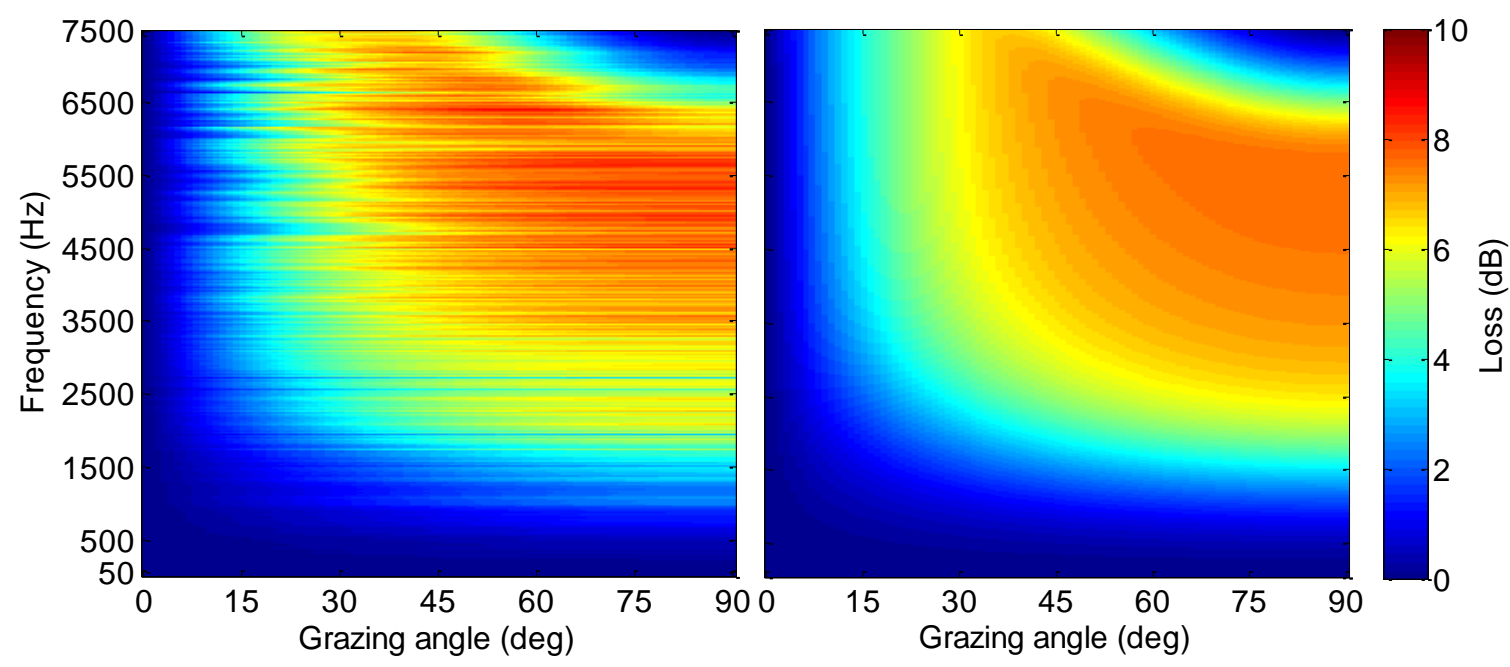

Figure 20. Beamformed BL from GLASS'12 data (left), and the case resulting from the exhaustive search (right).

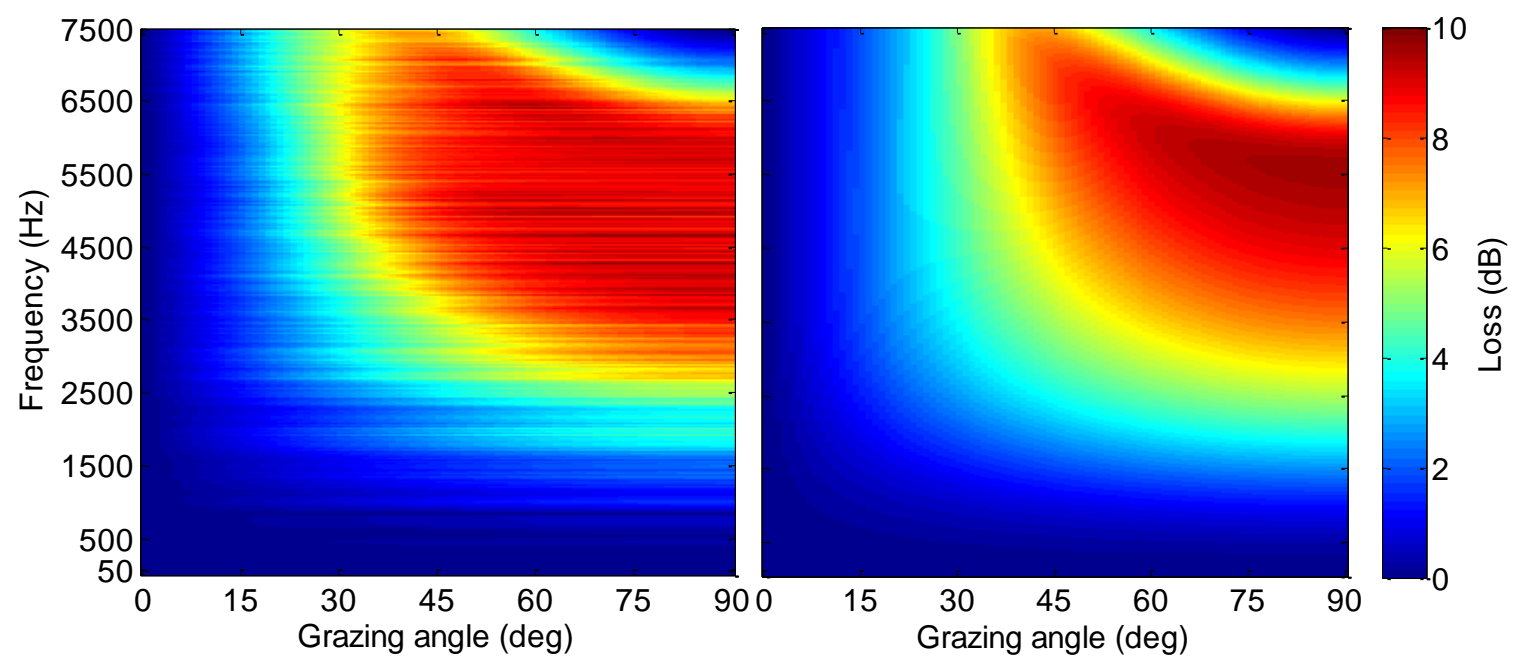

Figure 21. Beamformed BL from GLASS'13 data (left), and the case resulting from the exhaustive search (right).

For the GLASS'12 experiment, seabed properties deduced from cores collected on site showed a muddy bottom, with sound speed around $1510 \mathrm{~m} / \mathrm{s}$ and density in the range of $1600-1800 \mathrm{~kg} / \mathrm{m}^{3}$. Although these measurements are rigorously representative 
only of the upper $1 \mathrm{~m}$ of the seabed, whereas the array-deduced measurement can be influenced by bottom material at greater depths, they represent a basic benchmark for this study. The results shown in TABLE IV are representative of what was obtained examining a number of data snapshots from the sites, and show that the sound speed for GLASS'12 is in very good agreement with core measurements. The overestimation of the density is believed to be a consequence of the low surface-generated noise level from that experiment, which can cause an excessively low estimate of the bottom loss, which the algorithm attempts to "justify" by introducing higher density values. For the GLASS'13 experiment, no ground-truth data are available, but the sound-speed and density values to which the algorithm converges are sensible. In general, the search seems to place properly the critical angle (which is controlled by the sound speed) and the cutoff frequency below which the array does not measure any bottom loss. Given the nature of the cost function, it is reasonable to imagine that these two features are the ones driving the algorithm.

\subsubsection{Application of the HR-BL algorithm}

The estimated bottom-loss plots in Figure 19 show the limitations that one could expect from the simulation experiment shown in Figure 3: The limited angular resolution causes an uncertain location of the critical angle, significant underestimation of the bottom loss in the lower frequency range, and possibly loss of the striations indicating the presence of layers in the bottom. The HR-BL algorithm presented in Section 5 has proved to improve the angular resolution of relatively short arrays, and now the very short GLASS array offers a particularly hard test. 
Figure 22 shows the results of applying HR-BL to the same data that generated Figure 19. In both cases, the new plots present features that one would expect from improved angular resolution, based on the analysis in Section 5: Generally higher bottom-loss levels, which help "extend" the plot significantly towards lower frequencies, and a sharper transition at the critical angle.
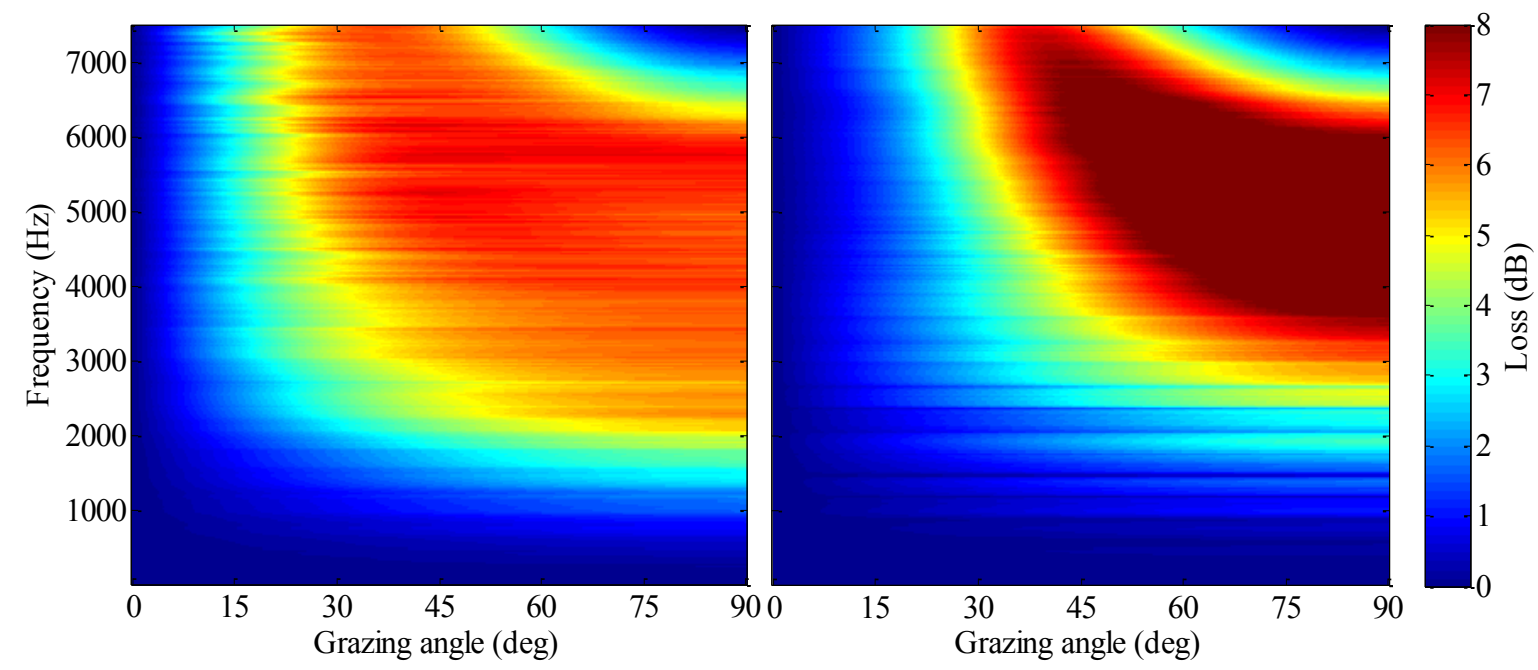

Figure 22. Estimated bottom loss from GLASS12 (left) and GLASS13 (right) using the HR-BL algorithm.

Unfortunately, data from a longer array are not available in either case, so one cannot validate these results against any "ground truth". However, the consistency with what shown in Section 6 makes these results appear sensible.

\subsection{Results from the Recognized Environmental Picture experiment}

The REP14-MED sea trial was conducted in the Sardinian Sea (Western Mediterranean) in June 2014, and coordinated by the CMRE as part of a series of multinational sea experiments. Two vessels participated in the campaign: the NATO Research Vessel Alliance, and the Research Vessel Planet of the German Navy. The 
experiments involved the collection of both physical oceanography and underwater acoustics data.

The NEAR Lab was involved both in the collection and in the processing of marine ambient-noise data recorded by two CMRE hydrophone arrays deployed during the campaign. The objective of the non-anthropogenic ambient-noise experiments was to validate seabed characterization by using short hydrophone arrays with a dimension suitable for installation on gliders and autonomous underwater vehicles.

In order to fulfill the objective, a newly developed prototype array (called HYDRA) was deployed, consisting of eight hydrophones positioned in a line and mounted on a ridged pole. The spacing of the individual hydrophones was adjustable from $0.15 \mathrm{~m}$ (design frequency of $5 \mathrm{kHz}$, total length of $1.05 \mathrm{~m}$ ) to $0.30 \mathrm{~m}$ (design frequency of $2.5 \mathrm{kHz}$, total length of $2.10 \mathrm{~m}$ ). The reference array (called SLIVA) had 32 hydrophones positioned at a fixed spacing of $0.18 \mathrm{~m}$ (design frequency of $4.167 \mathrm{kHz}$.) The arrays were bottom moored a few hundred meters apart with a distance from the seabed to the first hydrophone of approximately $20 \mathrm{~m}$ in a water depth of $170 \mathrm{~m}$. The weather conditions during the ambient noise measurements were calm to moderate with white caps visually observed.

Although data from both arrays were processed, in this dissertation only results from the SLIVA array are shown, because the spacing of this array is very close to that of the HYDRA prototype, and the 32 elements allow one to carry out a comparative study, where only part of the array can be selected, and its performance compared against the full array, in a fashion similar to what has been shown in Section 6 . 
The SLIVA array was deployed at a single site during REP14-MED, and processing of ambient-noise data for the full array produces the bottom loss shown in Figure 23. The appearance of this plot is markedly different from that of other plots seen before, particularly for the presence of the large area of low loss between 1 and $2 \mathrm{kHz}$, below $60^{\circ}$. The origin of this peculiar behavior is unknown at present, but its presence does not preclude the use of these data for the purpose of evaluating the performance of the algorithms described in this study. Furthermore, there appears to be some evidence of striations similar to those seen in other datasets, indicating the presence of layering in the bottom.

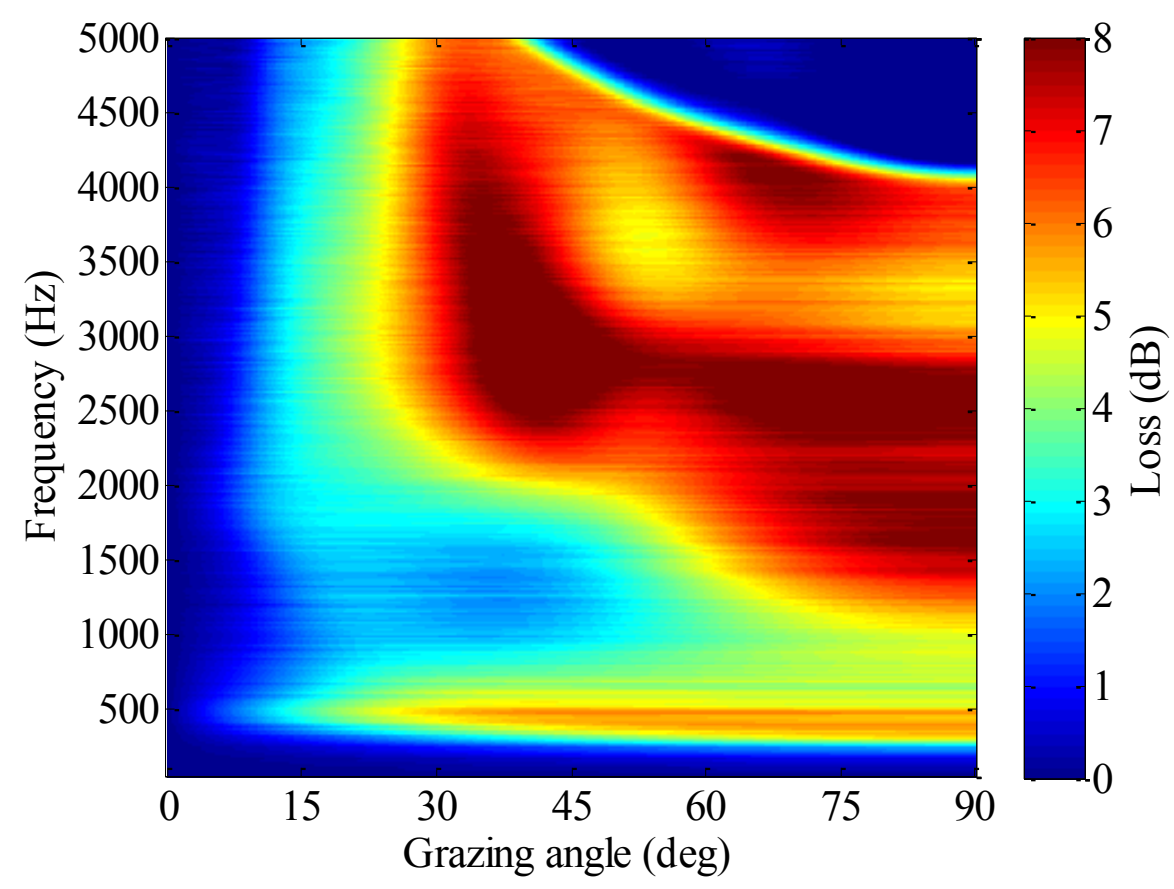

Figure 23. SLIVA data: Estimated bottom loss using the full 32-element array. 

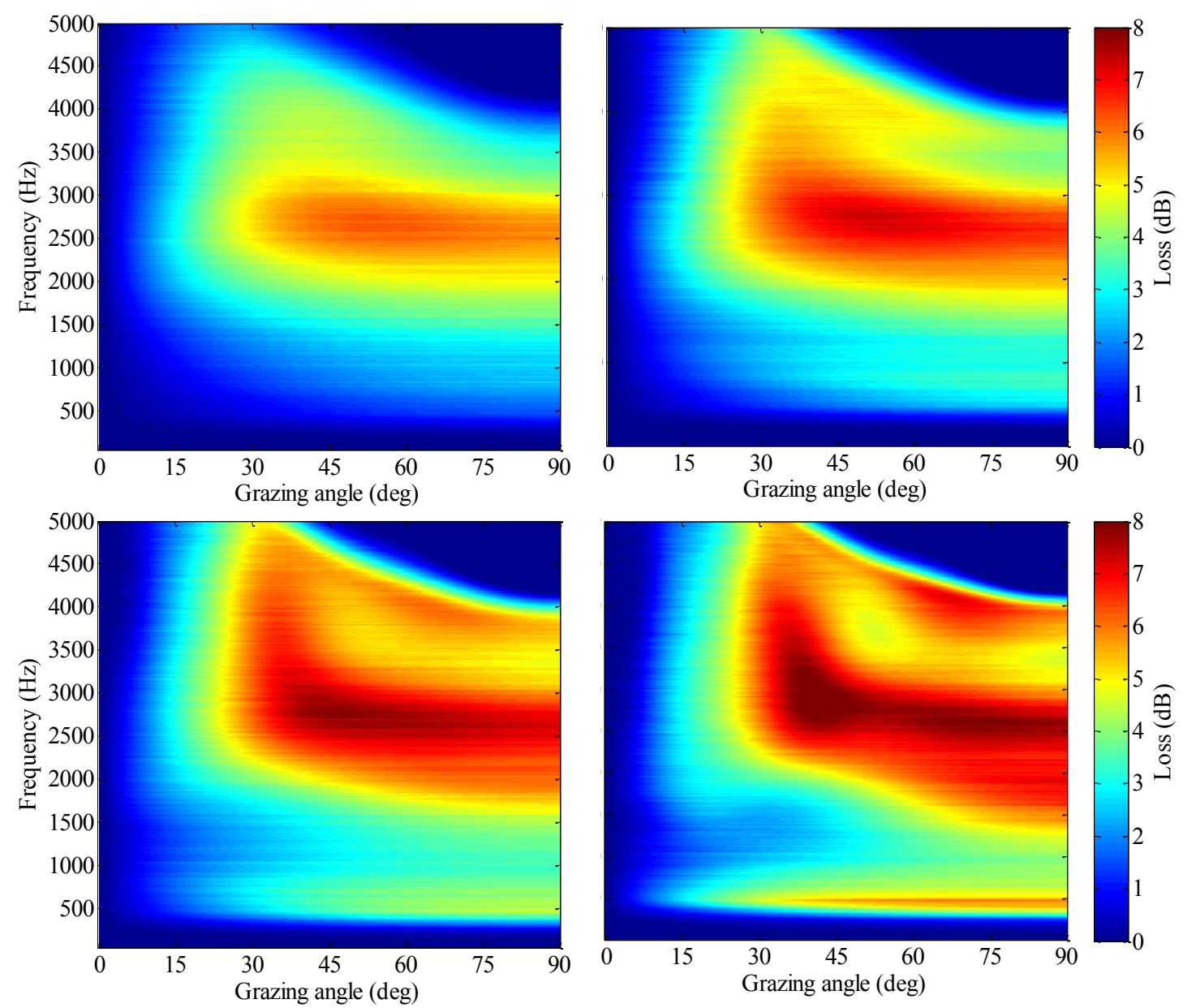

Figure 24. SLIVA data: Estimated bottom loss using 8 (top), and 16 (bottom) elements of the array, and CBF (left) and HR-BL (right).

In Figure 24, the estimated bottom loss from 8 and 16 elements of the array is computed using the original technique by Harrison and Simons, and the HR-BL algorithm. Similarly to what was done in Figure 10, in order to process the entire frequency range in the same way, a Hanning window was applied to the data. This is not the most advantageous for the HR-BL algorithm, but even so, the plots show improvement in both cases, along the same lines as described in Section 6 . 


\subsection{Summary}

The analysis conducted in this study of the performance of the GLASS array for the purpose of passive bottom-loss estimation indicates that the task presents challenges of a unique nature. This work, involving simulation, modeling, and data analysis, represents the first investigation of the impact of these particular array configurations on the results obtained by Harrison and Simons' technique, as well as the first analysis of the GLASS'13 dataset for this purpose. The encouraging results are that array tilt, when limited to less than $15^{\circ}$, does not appear to impair the bottomloss measurement, and that the results do appear to contain meaningful information on the bottom.

The array can be used to distinguish between different types of bottom, but the limits imposed by its much reduced length cause a significant loss in angular resolution, with the consequences expected on the quality of the estimated bottom loss. In order to successfully migrate Harrison and Simons' technique from moored/drifting arrays to small AUV mounted arrays, improved angular resolution must be achieved, and, despite the lack of ground truth, the results achieved by the HR-BL algorithm are consistent with such an improvement.

This section also presented the application of HR-BL to data collected by the SLIVAI array during the REP14-MED measurement campaign. Unlike the GLASS array, the SLIVA-I array, with its 32 elements, affords the comparison of the performance of HRBL between the results obtained by a sub array of a length deployable on a small AUV (8 sensors were chosen in this case, for a total length of $1.26 \mathrm{~m}$ ) and a longer sub array 
(16 elements were chosen in this case, for a total length of $2.70 \mathrm{~m}$ ). The results show that the HR-BL algorithm, as expected, can improved the BL estimate and recover some of the features lost when moving from a longer array to a shorter one. 


\section{Frequency based Coherence-function extension}

The HR-BL algorithm, illustrated in Section 6, enhances the angular resolution of the BL estimated from array data by exploiting specific properties of the ambient-noise vertical coherence function to remove some undesirable effects of conventional beamforming. ${ }^{34}$ Another recent study proposed to employ existing algorithms for the extrapolation of band-limited signals to reconstruct the noise coherence function of a longer array, starting from data measured by a shorter array. The extrapolation results appeared promising, but the potential of this technique for bottom-loss estimation has not been investigated yet. ${ }^{35}$

In this section, the idea of overcoming the limitations of short arrays by synthesizing the coherence function of a longer array is treated with the specific purpose of improving the performance of bottom-loss estimation (particularly the angular resolution) through Harrison and Simons' technique. However, instead of applying extrapolation algorithms, the proposed technique uses data measured at different frequencies by the hydrophones, to approximate the coherence function at sensors located beyond the physical length of the array. The treatment begins by describing the algorithm, and then proceeds to justify its use by means of both theoretical and empirical arguments.

\subsection{Algorithm}

This section introduces an algorithm for extending the coherence function beyond the physical length of the array, which in the remainder of this dissertation will be called 
"frequency based extension" (FBE). The algorithm is computationally simple, and makes a more efficient use of the frequency band available to modern acquisition systems, which often extends well beyond the array design frequency.

It has already been shown in this dissertation that the performance of an array of sensors in noise depends upon the accuracy of the estimate of the CSD matrix - i.e., the second order statistics of the noise field at the sensors (see Section 3) - and that this is modeled in physics by the spatial coherence function $C_{\omega}^{\prime}(z)$, defined in Eq.(2) and Eq.(3). The idea at the basis of FBE is as follows: Given two frequency values $\omega_{1}>\omega_{0}$, and two spacing values $z_{1}>z_{0}$, an estimate of the coherence function $C_{\omega_{0}}^{\prime}\left(z_{1}\right)$ can be obtained, if an estimate of $C_{\omega_{1}}^{\prime}\left(z_{0}\right)$ is available (e.g., from measured data), as long as the condition:

$$
\omega_{1} z_{0}=\omega_{0} z_{1}
$$

(or, equivalently, $z_{0} / \lambda_{1}=z_{1} / \lambda_{0}$ ) is met. From a practical standpoint, it is convenient to implement the algorithm by choosing $z_{0}=(M-1) d$ (where $M$ is the total number of elements in the array), i.e., the total length of the array. Then, the (extended) value of the function at $z_{1}=n d>z_{0}-$ where $n$ is an integer such that $n \geq M-$ can be obtained by assuming:

$$
\begin{aligned}
C_{\omega_{0}}(n d) & =C_{\omega_{1}}[(M-1) d], \\
\omega_{1} & =n \omega_{0} /(M-1) .
\end{aligned}
$$


The base spacing $z_{0}$ is set to the maximum value available on the physical array on purpose, in order to minimize the difference between $\omega_{0}$ and $\omega_{1}$, for reasons that will be clarified below. If $C_{\omega_{1}}^{\prime}\left(z_{0}\right)$ is obtained from data measured by an array, then this algorithm can be used to estimate the coherence function for spacing values greater than the physical length of the array. This bare definition of the FBE algorithm is integrated in the following sections with examples of its application to simple cases, then the treatment proceeds to justify its use by means of both theoretical and empirical arguments.

\subsection{Modeling and application: Halfspace bottom}

It is easier to start discussing the FBE algorithm by considering the simple case of a bottom composed of a single material of constant acoustic properties, extending indefinitely in depth - this bottom type will be referred to as "halfspace". The starting point is provided by the integral expression derived by Harrison for the unnormalized noise vertical coherence, already introduced in Eq.(18) and repeated here for convenience:

$$
\begin{aligned}
C_{\omega}(z)= & \int_{0}^{\pi / 2} \frac{2 \pi\left(c_{r} / c_{s}\right) \sin \theta \cos \theta}{1-R_{s}(\theta) R(\theta) e^{-a s_{c}(\theta)}} \\
& \left\{e^{i(\omega / c) z \sin \theta} e^{-a s_{p}}+R(\theta) e^{-i(\omega / c) z \sin \theta} e^{-a\left[s_{c}(\theta)-s_{p}(\theta)\right]}\right\} d \theta .
\end{aligned}
$$

Since the reflection coefficient of a halfspace bottom is independent of frequency, ${ }^{2}$ if one neglects the frequency dependence of $a$ (an acceptable assumption, as shown later), in this case the integrand of $C_{\omega}(z)$ does not depend on $\omega$ and $z$ separately, but 
rather on the term $\omega z / c=2 \pi z / \lambda$, where the familiar ratio of sensor spacing to wavelength appears. This is confirmed by the example provided in Figure 25 and Figure 26: Both plots show the normalized coherence function computed at selected frequency values using Eq.(45) and Eq.(3), for a halfspace bottom (see case HS in TABLE V for the physical properties of the water column and the bottom.) Each curve is plotted using 391 points over $0 \leq z \leq 5.85 \mathrm{~m}$, corresponding to an inter-sensor spacing of $0.015 \mathrm{~m}$. The markers show the positions of sensors number 10,20 , and 40 in an array of spacing $d=0.15 \mathrm{~m}$ (this value is used in the simulations shown later in Section 8.4).

In Figure 25, the curves are plotted as a function of sensor spacing $z$, and show the familiar decay along the horizontal axis, with more oscillations included over the array aperture as the frequency increases. However, the dependence of the coherence on the $z / \lambda$ ratio is better illustrated by the $C_{\omega}^{\prime}(z / \lambda)$ curves shown in Figure 26, where, given the quantity on the horizontal axis, at a lower frequency two consecutive points of a curve are closer than they are at a higher frequency. The plots show that, aside from the slight amplitude differences due to the inclusion of volume attenuation in Eq.(45) - which is at the basis of the model used to generate these plots - both the real and the imaginary part of $C_{\omega}^{\prime}(z / \lambda)$ overlap almost perfectly, regardless of frequency. However, due to the difference in wavelength, the curves at higher frequencies extend farther to the right on the horizontal axis than those at lower frequencies. Using Figure 26 for reference, understanding how the FBE algorithm works becomes straightforward, in this case: For example, the maximum spacing for 
a 10 -element array is $z_{0}=1.35 \mathrm{~m}$, and an additional ("nonphysical") sensor number 11 would be at $z_{1}=1.50 \mathrm{~m}$ from sensor number 1 . If one assumes $f_{0}=\omega_{0} / 2 \pi=$ $1 \mathrm{kHz}$, then $z_{1} / \lambda_{0}=1$, and Eq.(43) yields $f_{1}=(1.50 / 1.35) f_{0}=1.111 \mathrm{kHz}$. The point corresponding to $z / \lambda=1$ on the $1.111 \mathrm{kHz}$ curve is then used to estimate the coherence at the position of the "nonphysical" sensor number 11 on the $1 \mathrm{kHz}$ curve. The maximum available value for $z_{0}$ is chosen on purpose in this example, so as to minimize the difference between $\omega_{0}$ and $\omega_{1}$, ensuring that the error between the two $C_{\omega}^{\prime}(z / \lambda)$ curves is minimized.

TABLE V. Water-column and bottom properties for the simulated cases; $\Delta$ is the layer thickness, $\rho$ is the density, $\alpha_{c}$ is the compressional volume attenuation, and $\lambda$ is the wavelength.

\begin{tabular}{lcccc}
\hline & $\Delta(\mathrm{m})$ & $c_{p}(\mathrm{~m} / \mathrm{s})$ & $\rho\left(\mathrm{kg} / \mathrm{m}^{3}\right)$ & $\alpha_{c}(\mathrm{~dB} / \lambda)$ \\
\hline \multicolumn{1}{c}{ Water } & 170 & 1500 & 1000 & $1 \mathrm{e}-4$ \\
\hline \multicolumn{1}{c}{ HS } & $\infty$ & 1565 & 1500 & 0.2 \\
\hline 1L - Layer & 0.5 & 1650 & 1500 & 0.2 \\
1L - Halfspace & $\infty$ & 1700 & 2000 & 0.5 \\
\hline 2L - Layer \#1 & 0.5 & 1565 & 1500 & 0.2 \\
2L - Layer \#2 & 3 & 1625 & 1700 & 0.3 \\
2L - Halfspace & $\infty$ & 1800 & 2000 & 0.5 \\
\hline \hline
\end{tabular}



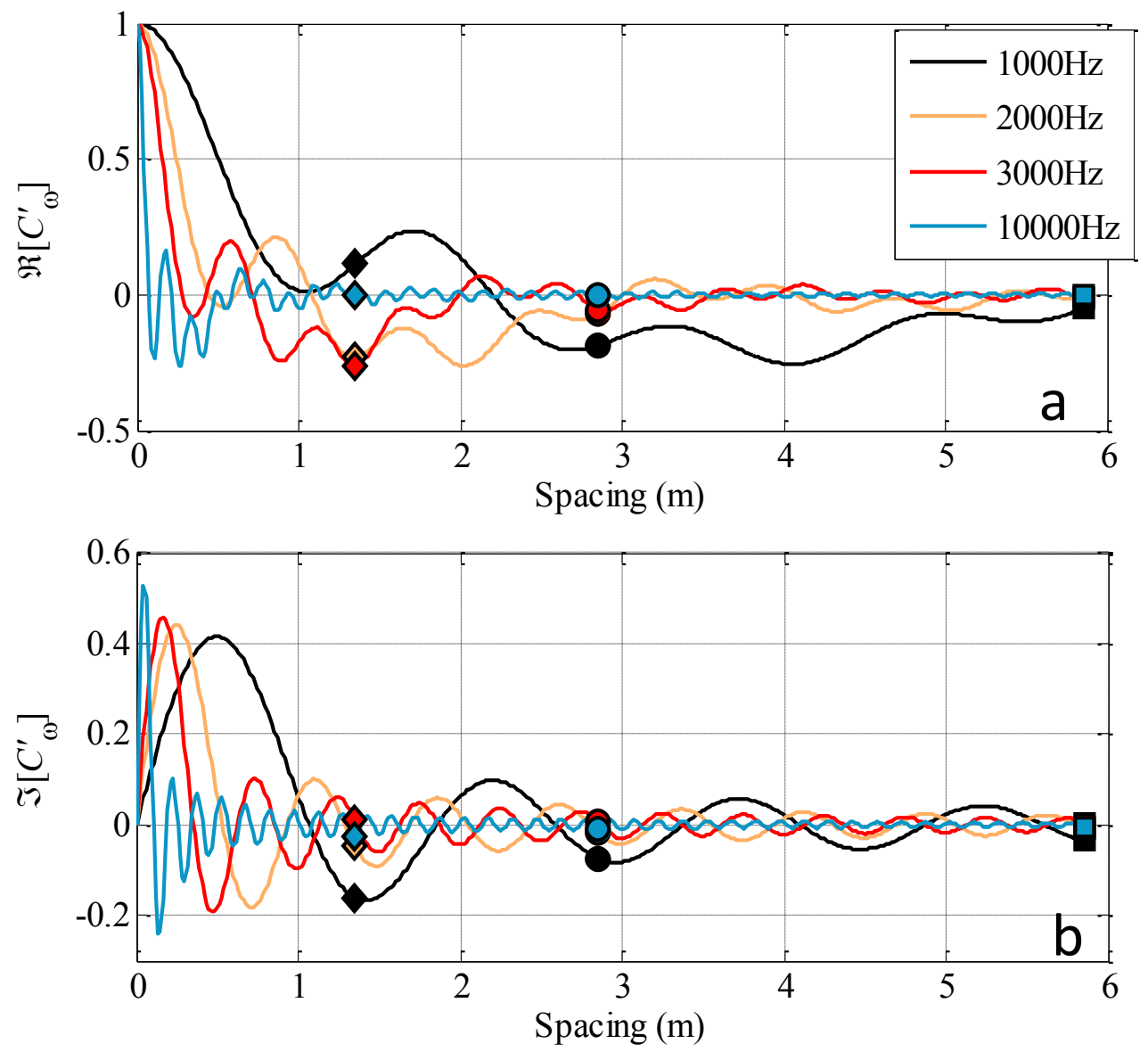

Figure 25. Halfspace bottom (case HS): Normalized coherence-function real (a) and imaginary (b) part at several frequencies, as a function of sensor spacing $z$. The markers indicate the positions of sensors number 10 (diamond), 20 (circle), and 40 (square) for an array of spacing $d=0.15 \mathrm{~m}$. 

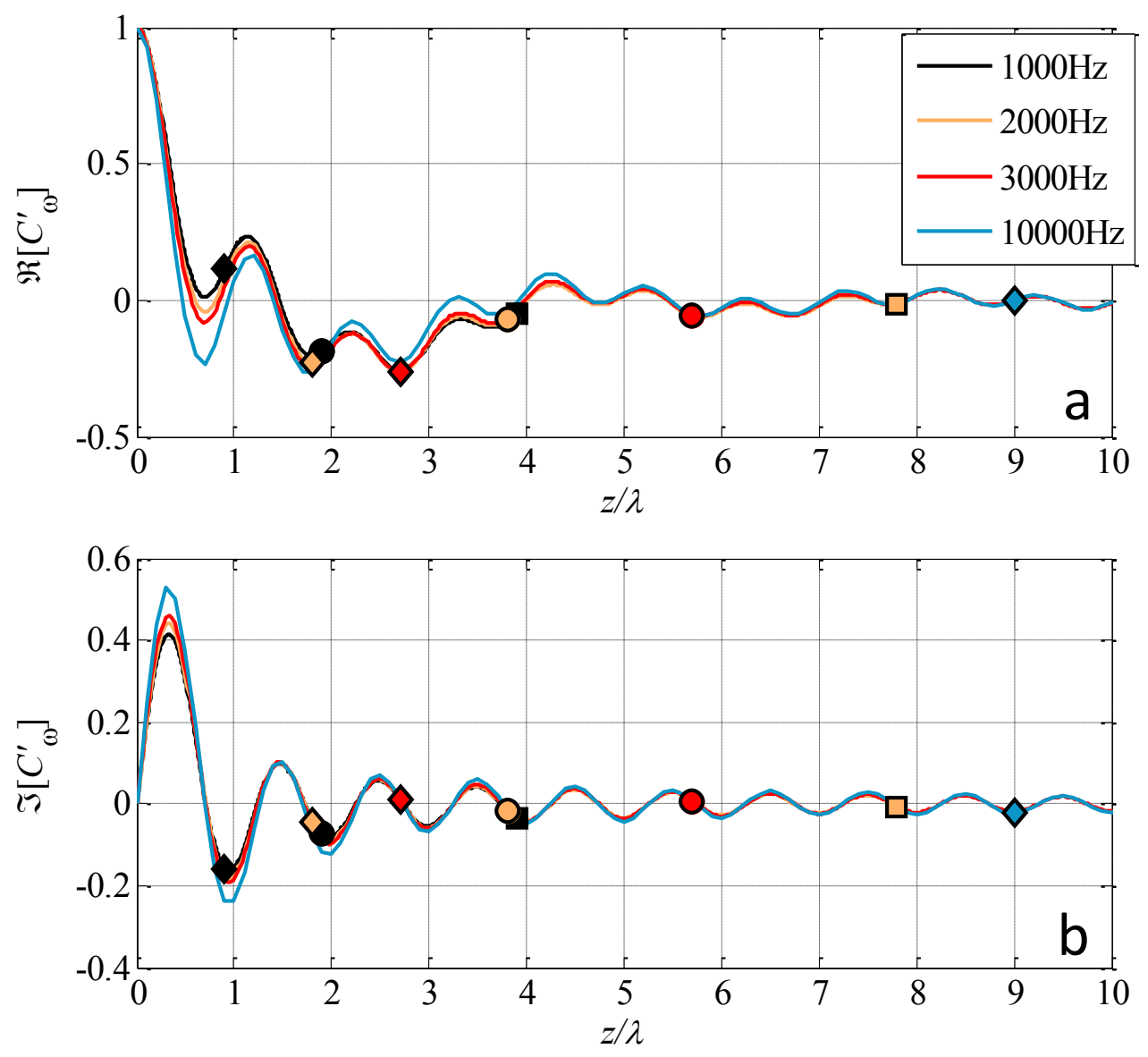

Figure 26. Halfspace bottom (case HS): Normalized coherence-function real (a) and imaginary (b) part at the same frequencies as in Figure 25, as a function of the $z / \lambda$ ratio. The markers indicate the same sensors as in Figure 25, but given the quantity on the horizontal axis, at a lower frequency two consecutive points of a curve are now closer than they are at a higher frequency, and the curve corresponding to a higher frequency reaches higher values on the horizontal axis. Note the almost perfect overlap of the curves. 


\subsection{Considerations on layered bottoms}

Although the treatment above relies on the fact that the bottom reflection coefficient is independent of frequency, use of the FBE algorithm prior to BL estimation (treated extensively in Section 8.4 and Section 8.5 below) improves the quality of the results also in the case of layered bottoms, where the frequency dependence of the reflection coefficient can be dramatic. The reason for this is not immediately apparent from theoretical models, which present $C_{\omega}(z)$ either in integral form, such as Eq.(45), or as a series expansion. ${ }^{67}$ The expression of $C_{\omega}(z)$ as the combination of a direct and an inverse Fourier transform between the hydrophone-spacing $z$ and the vertical wavenumber (and therefore, the frequency $\omega$ ) domains ${ }^{32,34}$ makes the connection between the two quantities explicit, but this fact alone does not fully explain why FBE is so effective in aiding BL estimation. An approach based on both theoretical and empirical considerations is proposed in this study.

As a starting point, the $C_{\omega}^{\prime}(z / \lambda)$ curves obtained from Eq.(3) and Eq.(45) for the layered bottom types 1L and 2L in TABLE V are shown in Figure 27 and Figure 28. One of the most remarkable features in the plots is that, while the real parts of $C_{\omega}^{\prime}(z / \lambda)$ appear to vary significantly between the two cases, the imaginary parts have a much more "regular" behavior, and appear to differ mostly in the amplitude of their oscillations. This can be explained starting from Eq. (45), and introducing the simplifying hypotheses of an isospeed water column with negligible volume attenuation $(a \approx 0)$, and a unit-value surface reflection coefficient $\left(R_{S} \approx 1\right)$, which yields the simplified expression: 


$$
C_{\omega}(z)=\int_{0}^{\pi / 2} \frac{\pi \sin 2 \theta}{1-R(\theta)}\left[e^{i(\omega z / c) \sin \theta}+R(\theta) e^{-i(\omega z / c) \sin \theta}\right] d \theta .
$$

Now, by expressing the exponentials in trigonometric form, one can separate the real and imaginary parts of the integral:

$$
\begin{aligned}
& \operatorname{Re}\left[C_{\omega}(z)\right]=\int_{0}^{\pi / 2} \pi \sin 2 \theta \frac{1+R}{1-R} \cos \left(\frac{\omega z}{c} \sin \theta\right) d \theta \\
& \operatorname{Im}\left[C_{\omega}(z)\right]=\int_{0}^{\pi / 2} \pi \sin 2 \theta \sin \left(\frac{\omega z}{c} \sin \theta\right) d \theta
\end{aligned}
$$

The most apparent feature in Eq.(47) is the absence of the reflection coefficient in $\operatorname{Im}\left[C_{\omega}^{\prime}(z / \lambda)\right]:$ In this simplified model, the imaginary part of $C_{\omega}^{\prime}(z / \lambda)$ is independent of the bottom reflection properties, and its dependence on $\omega$ is only present as the $\omega Z$ product. This conclusion is confirmed by Figure 27 and Figure 28, where the behavior of the imaginary part curves is perfectly analogous to that observed in the halfspace case (see Figure 26).

Furthermore, Eq.(47) indicates that the differences due to the bottom type should manifest themselves in the real part curves, which, in fact, show clear differences between the two cases. However, it should be noted that, even for these layered bottoms, if one considers two $\operatorname{Re}\left[C_{\omega}^{\prime}(z / \lambda)\right]$ curves corresponding to "close" frequency values, the points corresponding to the same $z / \lambda$ value on the two curves will be "close" too. In other words, although the curves can be proven theoretically to overlap perfectly (except for the frequency dependence of $a$ ) only for a halfspace bottom, they 
still appear to vary smoothly with frequency, in the case of layered bottoms. The results presented in Section 8.4 and Section 8.5 below confirm that this reasonable hypothesis holds, and that the FBE algorithm does help improve the BL estimates from short arrays.


Figure 27. Single layer over halfspace (case 1L): Normalized coherence-function real (a) and imaginary (b) part at several frequencies, as a function of the $z / \lambda$ ratio. The markers are positioned as in Figure 26. 

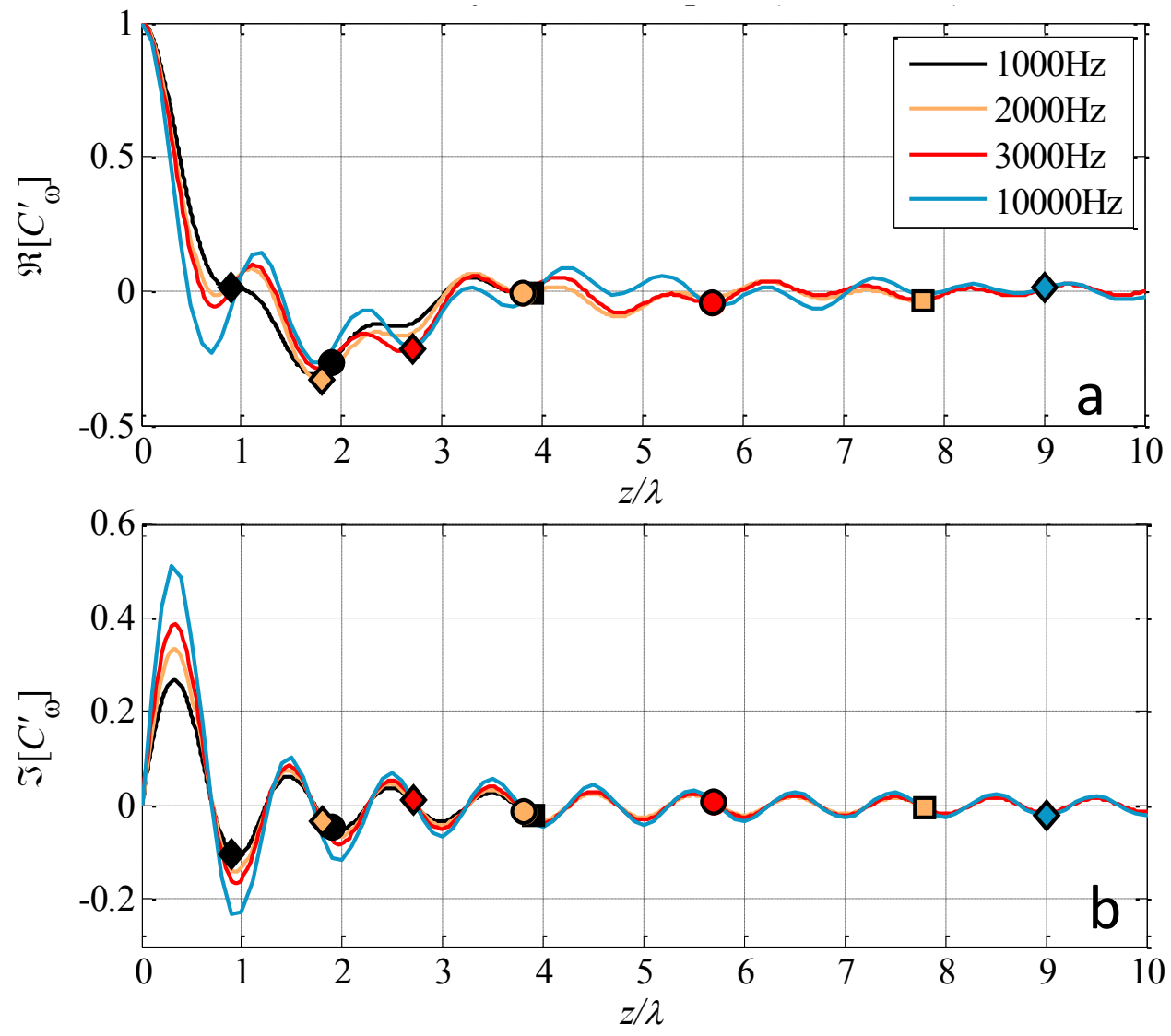

Figure 28. Double layer over halfspace (case 2L): Normalized coherence-function real (a) and imaginary (b) part at several frequency values, as a function of the $z / \lambda$ ratio. The markers are positioned as in Figure 26. 


\subsection{Application to bottom-loss estimation: Simulation}

Simulation can be useful at this point to investigate further the dependence of the coherence function on signal and array physical parameters. In the remainder of this chapter, results are presented from simulations run using OASN, the surface-noise module of the OASES ${ }^{77}$ package. By implementing a numerical solution to the full wave equation for range independent, stratified media - as opposed to implementing an analytical model - OASES produces directly the $\widehat{\mathbf{C}}_{\omega}$ matrix of Eq.(13), providing a more realistic approximation to what an estimate of the coherence function from measured data would be. For its simulation part, this study presents the application of OASN to the three different types of bottom already investigated above: Halfspace (identified by "HS"), single layer over halfspace ("1L"), and two layers over halfspace ("2L"). The bottom properties for each case are shown in TABLE V.

\subsubsection{Considerations on array configuration and the bandwidth of the estimated bottom loss}

Arrays suitable for AUV deployment should reasonably have a length not greater than $2 \mathrm{~m}$, but the applicability of Harrison and Simons' technique to such arrays, especially at frequencies below $10 \mathrm{kHz}$, has been shown to be unreliable, due to the severe deterioration of the beamformer's angular resolution. ${ }^{24}$ High resolution bottom-loss estimation (HR-BL) has recently shown that BL estimates from Harrison and Simons' technique can be improved, by replacing the CBF with a more sophisticated technique, which exploits the physical properties of the surface generated noise 
field. ${ }^{34}$ However, the challenge posed by BL estimation with AUV deployable arrays in the $500-5000 \mathrm{~Hz}$ frequency range makes any further performance improvement highly desirable.

As an example of an effective application of FBE, this section shows how it can improve significantly the performance of short arrays in passive bottom-loss estimation. The main advantage is the possibility of improving the grazing-angle resolution of the estimated bottom loss to a level that the original technique could only achieve by means of a longer array. A more subtle advantage is a more efficient use of the bandwidth of current acquisition systems, explained below.

The beamforming operation imposes a practical limitation on BL estimation: The upper limit on the frequency range over which data can be used (hereafter referred to as "array design frequency"). For conventional beamforming on a line array, this limit is determined by the inter-sensor spacing: The maximum frequency at which the array can operate as a directional antenna corresponds to a wavelength that is twice the spacing. For instance, assuming a sound speed in water of $1500 \mathrm{~m} / \mathrm{s}$, an array whose sensors are spaced $0.15 \mathrm{~m}$ has a design frequency of $5 \mathrm{kHz}$. The appearance of grating lobes in the beam pattern makes it impossible to estimate the bottom loss above the array design frequency.

However, with the sampling rates afforded by current acquisition systems, the array design frequency usually falls well below the Nyquist frequency, leaving a sizeable fraction of the data unused, for the purpose of bottom-loss estimation. In FBE, data from higher frequencies are used to estimate the noise spatial coherence 
function at a lower frequency, for values of the sensor spacing beyond the physical length of the array. As Eq.(44) shows, if one wants to double the number of sensors, and therefore the array length, and estimate the BL up to the array design frequency $f_{d}=c / 2 d$, it is necessary to have data available from the physical sensors up to a frequency that is roughly $2 f_{d}$. By doing so, the angular resolution of the bottom-loss estimate can be improved, often making use of data at frequencies otherwise not utilized for beamforming.

\subsubsection{Application to simulated data}

In this section, the application of FBE to passive bottom-loss estimation is investigated through simulation. This ensures the a priori knowledge of the bottom and the water column, a luxury that experiments on the field usually cannot afford, making it possible to compare the results to model-based predictions. Since the goal is bottom-loss estimation, in this study the reference is provided by a model, presented by Jensen et $a l_{.,}{ }^{2}$ that predicts the power reflection coefficient of a horizontally stratified fluid bottom of known physical properties, as a function of frequency and grazing angle.

For each of the cases introduced above, Jensen et al.'s model has been run to provide the predicted BL to be used as reference. For the HS case, the CSD matrices produced by OASN for a 24-element array with $0.15 \mathrm{~m}$ spacing have been processed to estimate the BL both by the HR-BL algorithm, and by CBF, as in Harrison and Simons' original technique. The latter procedure has been repeated using only the first 12 elements of the array, to show how the estimated BL is impacted by a 
significant reduction of the array length. The same 12-element CSD matrices have then been used to estimate the 12-point coherence function by diagonal averaging, ${ }^{34}$ and this has been extended as described in Section 8 up to the length of the original array, i.e. adding 12 points corresponding to "synthetic" sensors beyond the length covered by the "physical" sensors. In the examples presented in this section, the frequency domain has been sampled with 680 bins of $50 \mathrm{~Hz}$ width between $50 \mathrm{~Hz}$ and 34kHz. In general, the frequency value required to apply Eq.(44) will not fall at the center of one of the chosen frequency bins. The results presented in the remainder of this chapter have been obtained by simple linear interpolation of the coherence function between the closest available frequency bins. The extended coherence function has been used to build a Toeplitz CSDM, ${ }^{32,34}$ which has then been passed to the HR-BL algorithm to estimate the BL.

The results obtained by the procedure outlined above are shown in Figure 29. Panels $a$ and $c$ show the limitations imposed by beamforming: While the predicted BL is perfectly frequency independent, the 24-element beamformer - corresponding to a $3.45 \mathrm{~m}$ aperture - places the critical angle correctly only at the higher frequencies. With decreasing frequency, the beams become wider, and the decreased angular resolution causes an area of substantial underestimation, which extends to cover the entire grazing angle range at the bottom of the plot. The design frequency for this array is $5 \mathrm{kHz}$, and at this frequency, around normal incidence, the BL estimate drops to zero, due to the grating lobes that appear in the beam pattern. When the aperture is reduced to $1.65 \mathrm{~m}$ (12 elements), all the limitations indicated above are 
magnified. The last panel in Figure 29 shows the BL estimated with data from the same 12 sensors, but after extending the coherence function by FBE back to 24 elements: The plot shows a virtually complete recovery of the information lost by the shorter array.

Given the analysis presented in Section 8.2, the result above is not surprising, whereas for a layered bottom one could expect the more pronounced dissimilarity of the coherence-function real part to make the application of FBE more difficult. This has been preliminary investigated through a number of simulations, of which the results for cases $1 \mathrm{~L}$ and $2 \mathrm{~L}$, shown in Figure 30 and Figure 31 respectively, are presented as a sample. These results have been produced following the same procedure as the one described above for case HS.

For layered bottoms, the predicted BL presents much more structure than for a halfspace. Such structure can be rather fine, as shown, for instance, in case $1 \mathrm{~L}$ at the critical angle (the angle above which the BL becomes significant, Figure 30), and, in case $2 \mathrm{~L}$, in the thin striations overlapping with the three wide striations (Figure 31). The data being simulated, the BL estimated by Harrison and Simons' technique in these cases is very "clean", compared to what is usually observed when working with experimental data. Nevertheless, the 24-element CBF results present a significant underestimation of the BL at low frequencies, and in general the estimated BL appears to be a "smeared" version of the BL predicted by the Jensen et al.'s model. These effects are expected, as they are due to the finite angular resolution of the beamformer, and are accentuated when moving to the BL estimated by the half-length 
array (12 elements). The BL estimated by HR-BL using the data from the same 12 elements - after extending the estimated coherence function at the locations of 12 additional "synthetic" sensors - shows in both cases an almost complete recovery of the information lost by the 12-element $\mathrm{CBF}$, with a sharper critical-angle transition, better definition of the striations, and improved BL estimation at the lower frequencies. 

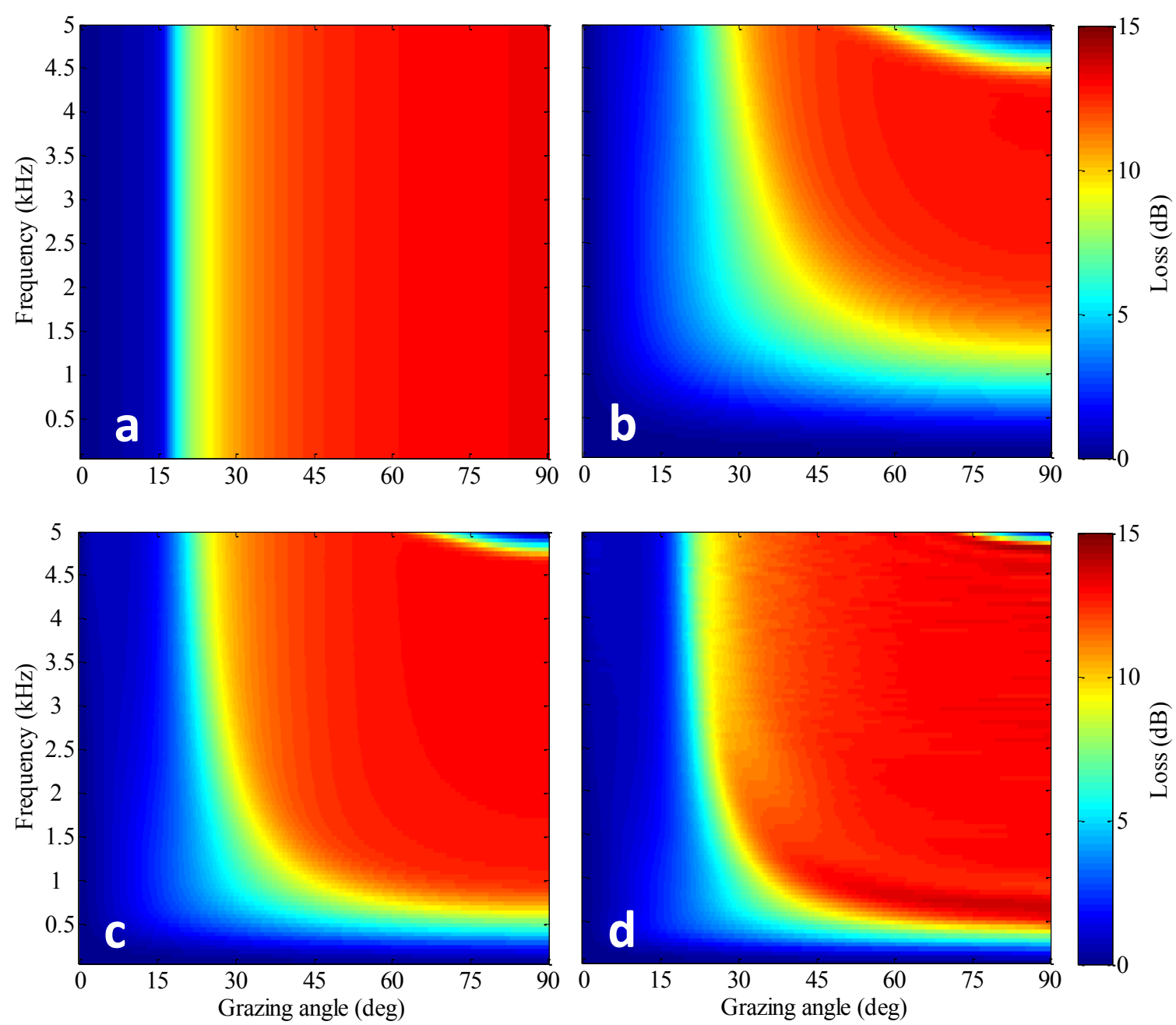

Figure 29. Halfspace bottom (case HS): BL predicted using the reflection coefficient given by Jensen et al.'s model (a); BL estimated from OASN data using CBF over 12 (b) and 24 (c) sensors, and BL estimated by HR-BL over 12 sensors extended to 24 by FBE (d). 

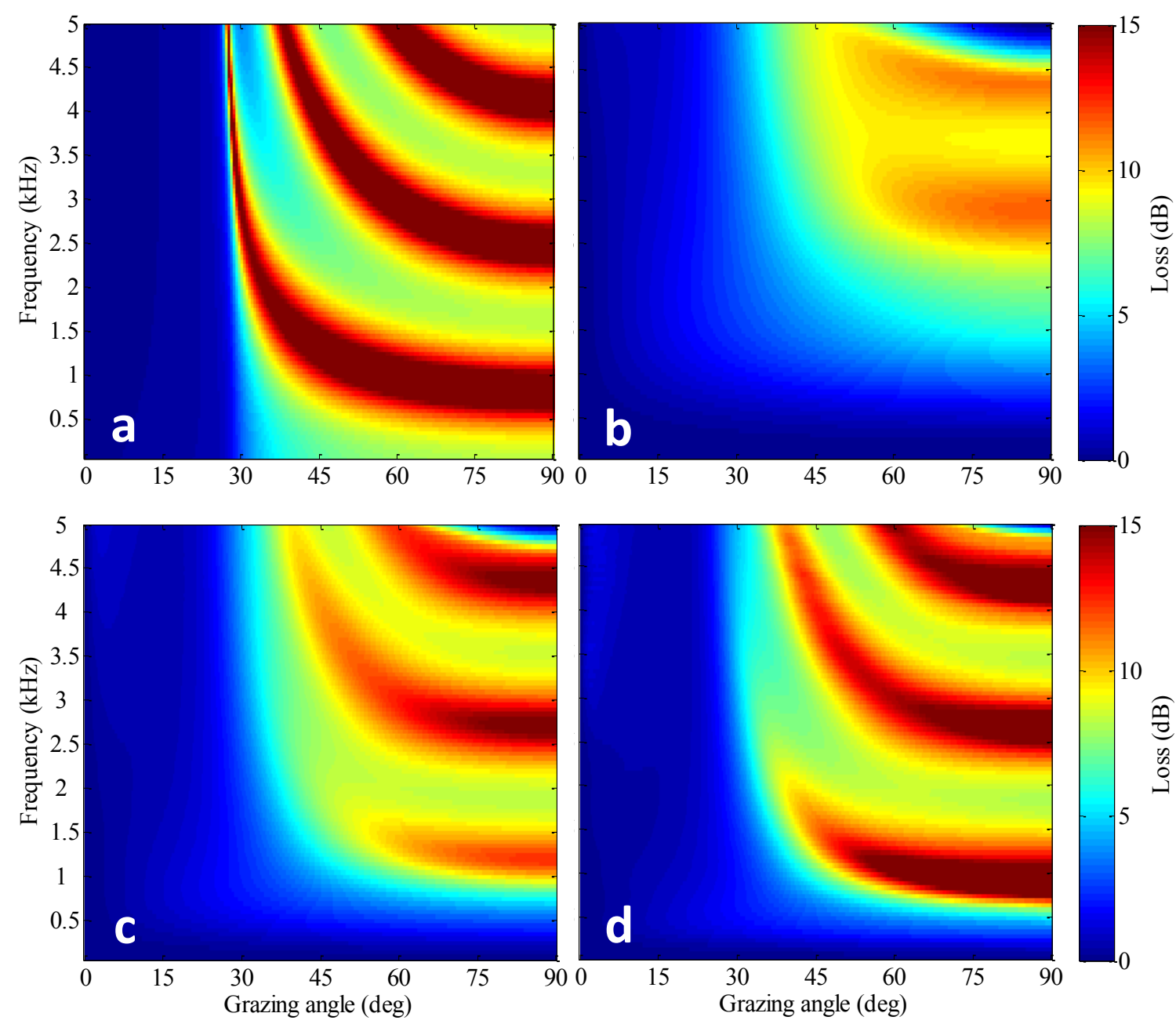

Figure 30. Single layer over halfspace (case 1L): BL predicted using the reflection coefficient given by Jensen et al.'s model (a); BL estimated from OASN data using CBF over 12 (b) and 24 (c) sensors, and BL estimated by HR-BL over 12 sensors extended to 24 by FBE (d). 

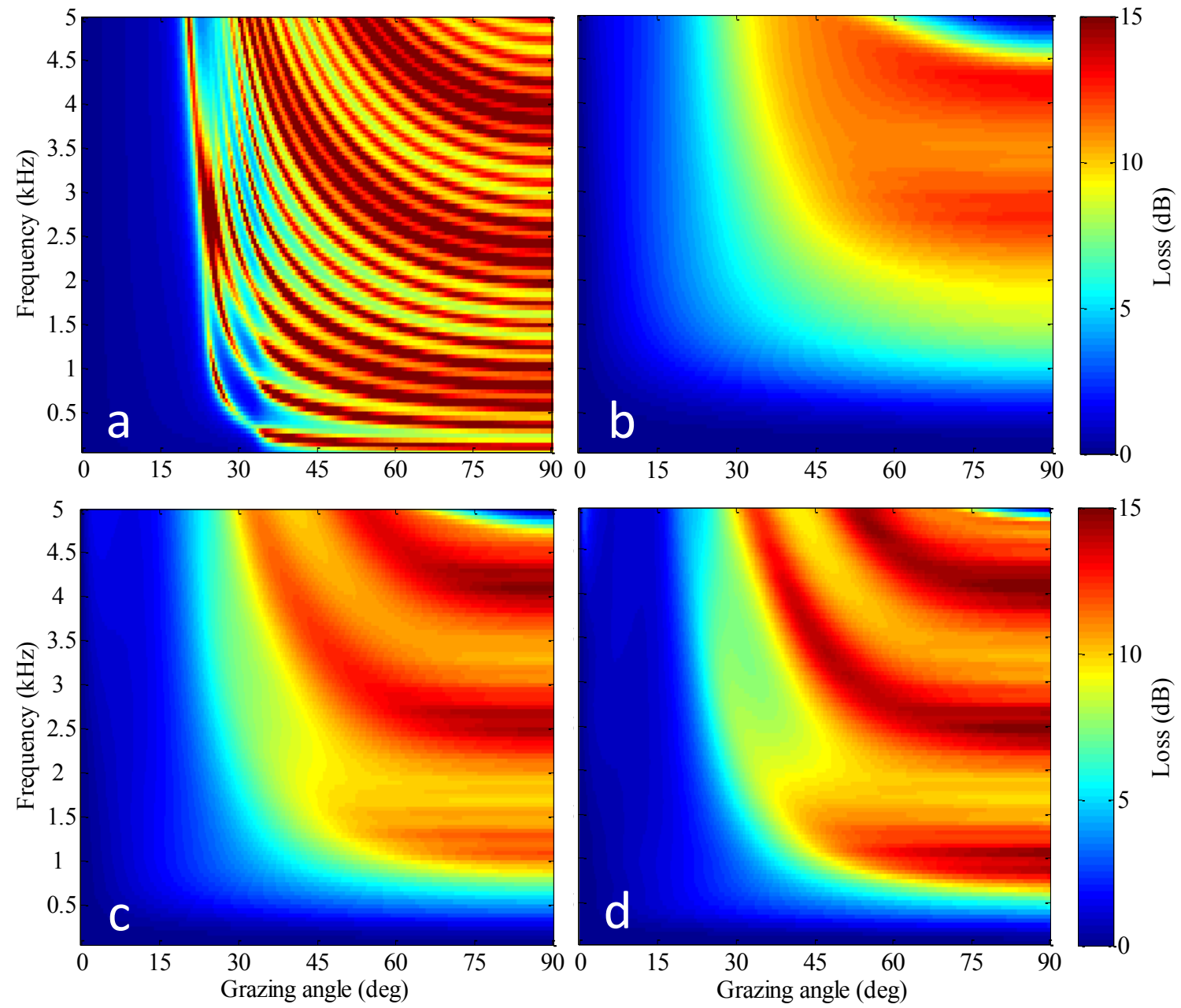

Figure 31. Double layer over halfspace (case 2L): BL predicted using the reflection coefficient given by Jensen et al.'s model (a). BL estimated from OASN data using CBF over 12 (b), and 24 (c) sensors, and BL estimated by HR-BL over 12 sensors extended to 24 by FBE (d). 


\subsection{Application to measured data}

This section presents the results of applying FBE to passive BL estimation from actual data measured in three different experiments at sea by the NATO-STO Centre for Maritime Research and Experimentation. The data refer to three different sites and arrays, and the dataset identifiers used in the remainder of this chapter, as well as the basic characteristics of each data set and array, are listed in TABLE VI. The MFA and VLA data are from the experimental campaign named Boundary 2003, while the SLIVA dataset was recorded during the REP14-MED experiment of 2014.

The emphasis in this study is in showing how FBE can improve the performance of a short array in BL estimation. For this reason, rather than comparing the results to a ground truth that in the case of the measured data is rather uncertain, the comparison is carried out between the full 32-element array, a subarray including only a subset of the original elements, and the same subarray extended to the original length by using FBE to estimate the coherence function at the location of the missing original sensors.

TABLE VI. Datasets and array basic features.

\begin{tabular}{cccccc}
\hline Dataset ID & $\begin{array}{c}\text { Num. of } \\
\text { elements }\end{array}$ & Spacing (m) & $\begin{array}{c}\text { Sampling freq. } \\
(\mathrm{Hz})\end{array}$ & $\begin{array}{c}\text { Design freq. } \\
(\mathrm{Hz}) @ \mathrm{c}= \\
1500 \mathrm{~m} / \mathrm{s}\end{array}$ & $\begin{array}{c}\text { Deploy } \\
\text { ment } \\
\text { type }\end{array}$ \\
\hline VLA-03 & 32 & 0.50 & 6000 & 1500 & Drifting \\
MFA-03 & 32 & 0.18 & 12000 & 4166 & Drifting \\
SLIVA-14 & 32 & 0.18 & 50000 & 4166 & Moored \\
\hline \hline
\end{tabular}


All the CSD matrices used in this study correspond to 5-minute averages of data. The plots in Figure 32, Figure 33, and Figure 34 compare the BL estimated by applying Harrison and Simons' original technique to data recorded over the full 32-element arrays of the Boundary experiment, and to a 20 -element subarray. In these examples, the magnitude of the extension (from 20 to 32 elements) is limited by the low Nyquist frequency, which in particular prevents the recovery of the BL estimate up to the design frequency $(4166 \mathrm{~Hz}$, with a sound speed of $1500 \mathrm{~m} / \mathrm{s}$ ) for the MFA dataset. In all cases the deterioration due to the loss in angular resolution is visible in the same terms as already described above for the synthetic data, and FBE coupled with HR-BL proves to be able to recover in the BL most of the information lost by applying the CBF to the same array, as in Harrison and Simons' original technique.

Furthermore, some high loss striations appear at very low grazing angles in the FBE result in Figure 32. It is unclear whether these represent an actual feature of the bottom, but analysis of the other panels in the figure, as well as Figure 33, shows that these features are present in the BL estimated by the other techniques, and are simply emphasized by the high resolution of the HR-BL algorithm.

Finally, the VLA results in Figure 34 may not appear to be as "dramatic" as the MFA ones; this is due to the nature of the data. The analysis of these data shows that the surface noise field is contaminated by other contributions, a circumstance to which the HR-BL algorithm is known to be sensitive. ${ }^{34}$ To alleviate the consequences of this, a Hanning taper was applied to the CSD matrix prior to HR-BL processing, a procedure that limits the performance of the algorithm. However, it should also be noted that 
although this low frequency array, with an inter sensor spacing of $0.5 \mathrm{~m}$, can be more challenging for FBE, it is also an unlikely candidate for AUV deployment.

Data-bandwidth limitation is a less important problem today, with data acquisition systems that are capable of much higher sampling rates. For instance, TABLE VI shows that the SLIVA array has the same spacing as the MFA array, but a much higher sampling rate. In Figure 35 the BL estimated using the full SLIVA array is compared to that obtained from an 8-element subarray (which could be used for AUV deployment), with the latter showing a significant loss of information. As the results show, in this case the sampling rate is high enough to "extend" the subarray back to 32 elements, therefore quadrupling the array length, up to the array design frequency. Even in this rather extreme attempt, the significant information recovery by the extended coherence function is apparent.

To conclude the experimental part of this study, it is important to stress that the quality of these results depends on the measured acoustic field being free of sources other than wind and wave noise. Furthermore, it should be noted that there can be features in the coherence function that do not manifest themselves in measurement if the array does not have an adequate length. In such cases, FBE may not recover such features, which may correspond to some details in the BL plot, but it will still provide an approximation to the general shape of the function, and its decay with increasing $z / \lambda$. 

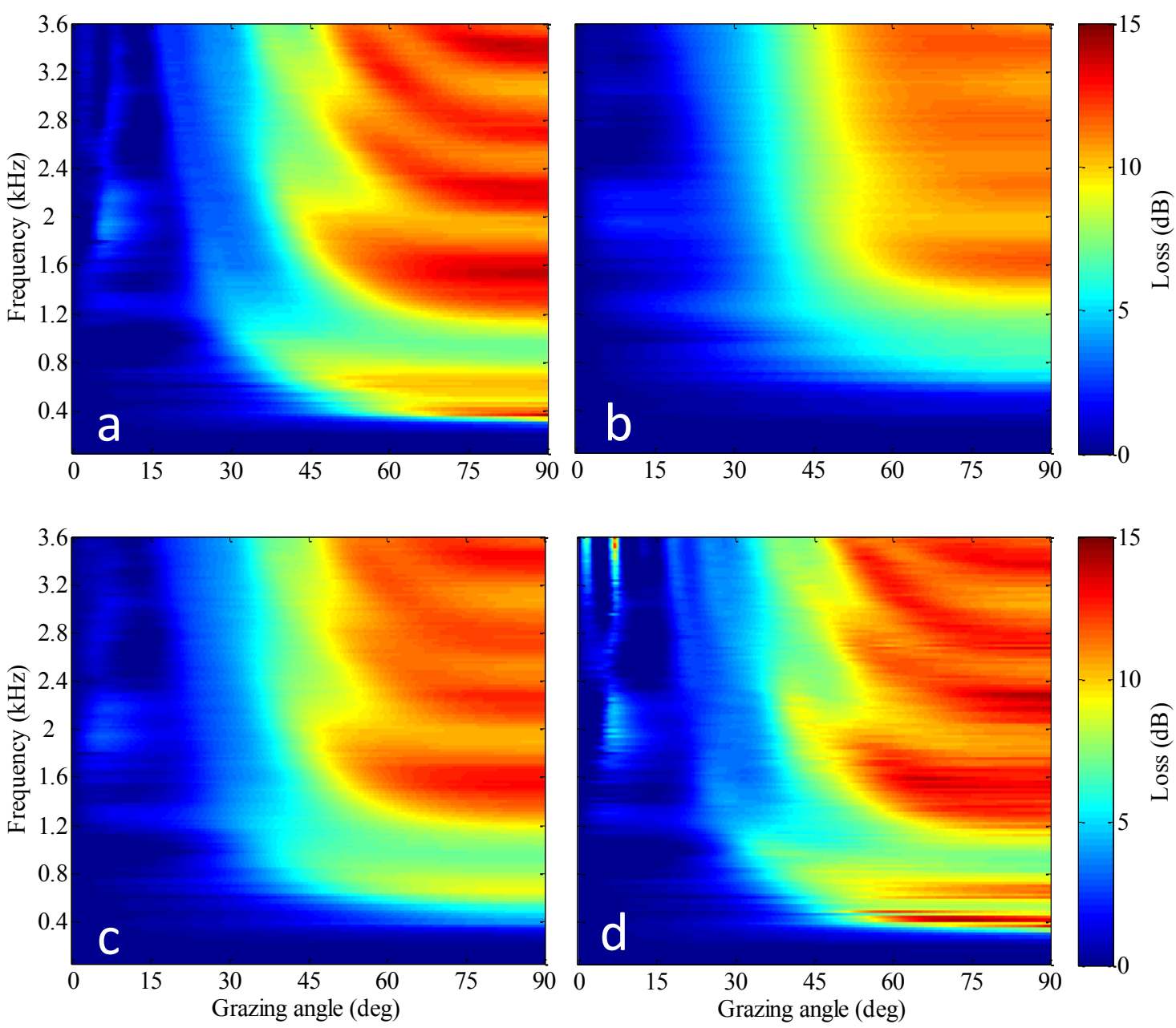

Figure 32. Boundary 2003 MFA data: BL estimated by HR-BL over 32 elements (a), by CBF over 20 (b) and 32 elements (c), and by HR-BL after extending the coherence function estimated from 20 sensors to 32 sensors by FBE (d). 

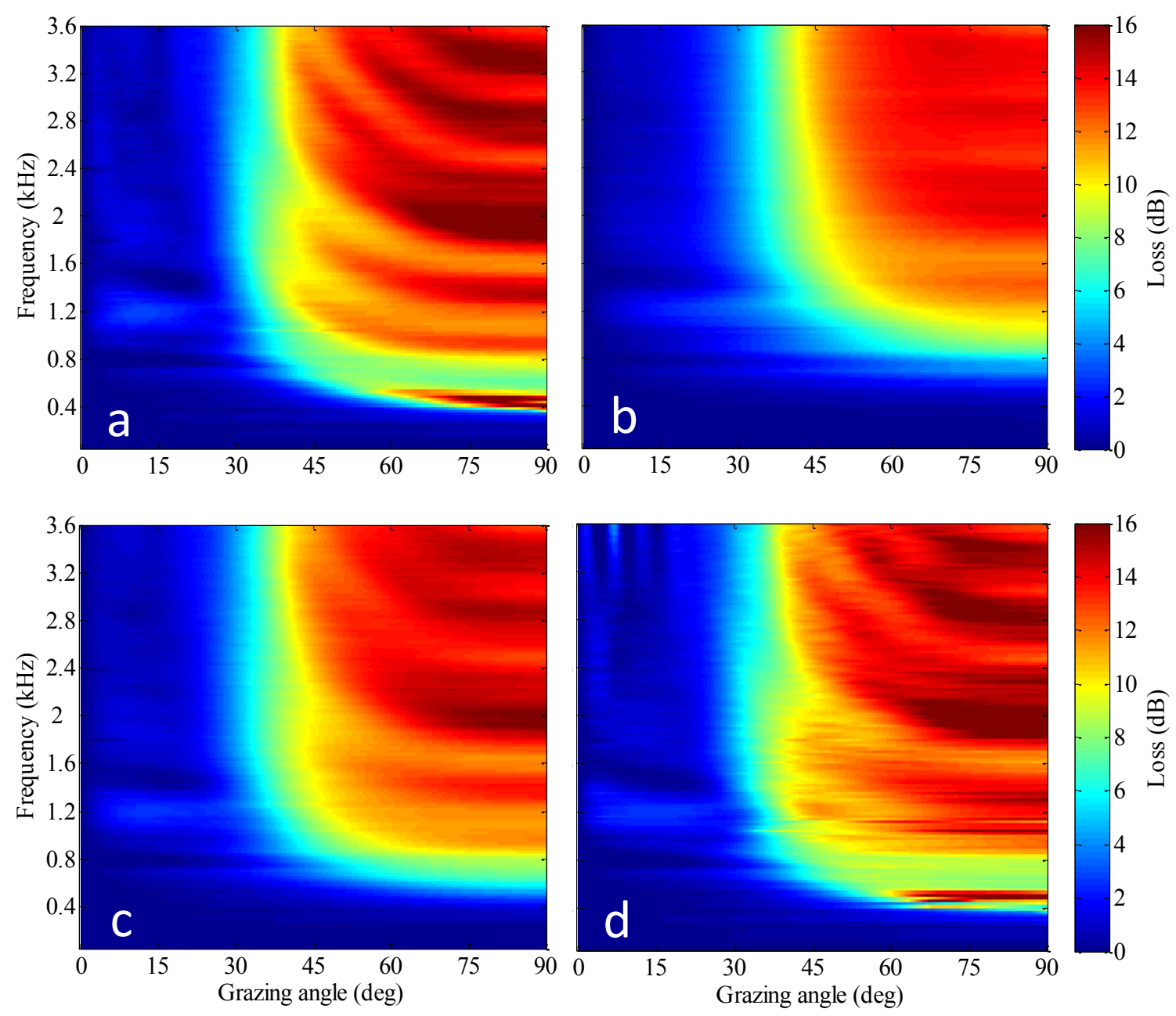

Figure 33. Boundary 2003 MFA data: BL estimated by HR-BL over 32 elements (a), by CBF over 20 (b) and 32 elements (c), and by HR-BL after extending the coherence function estimated from 20 sensors to 32 sensors by FBE (d). 

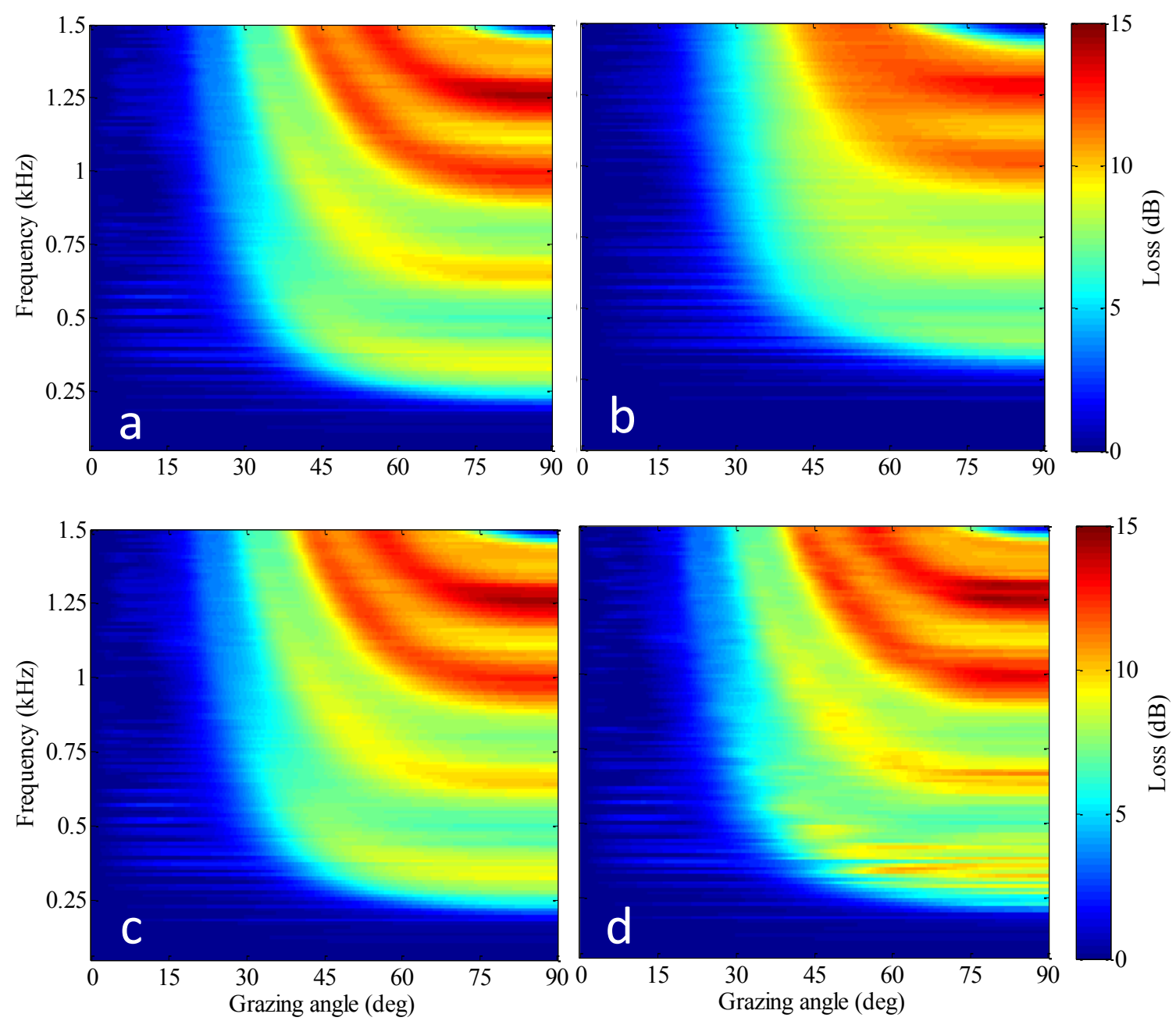

Figure 34. Boundary 2003 VLA data: BL estimated by HR-BL over 32 elements (a), by CBF over 20 (b) and 32 elements (c), and by HR-BL after extending the coherence function estimated from 20 sensors to 32 sensors by FBE (d). 

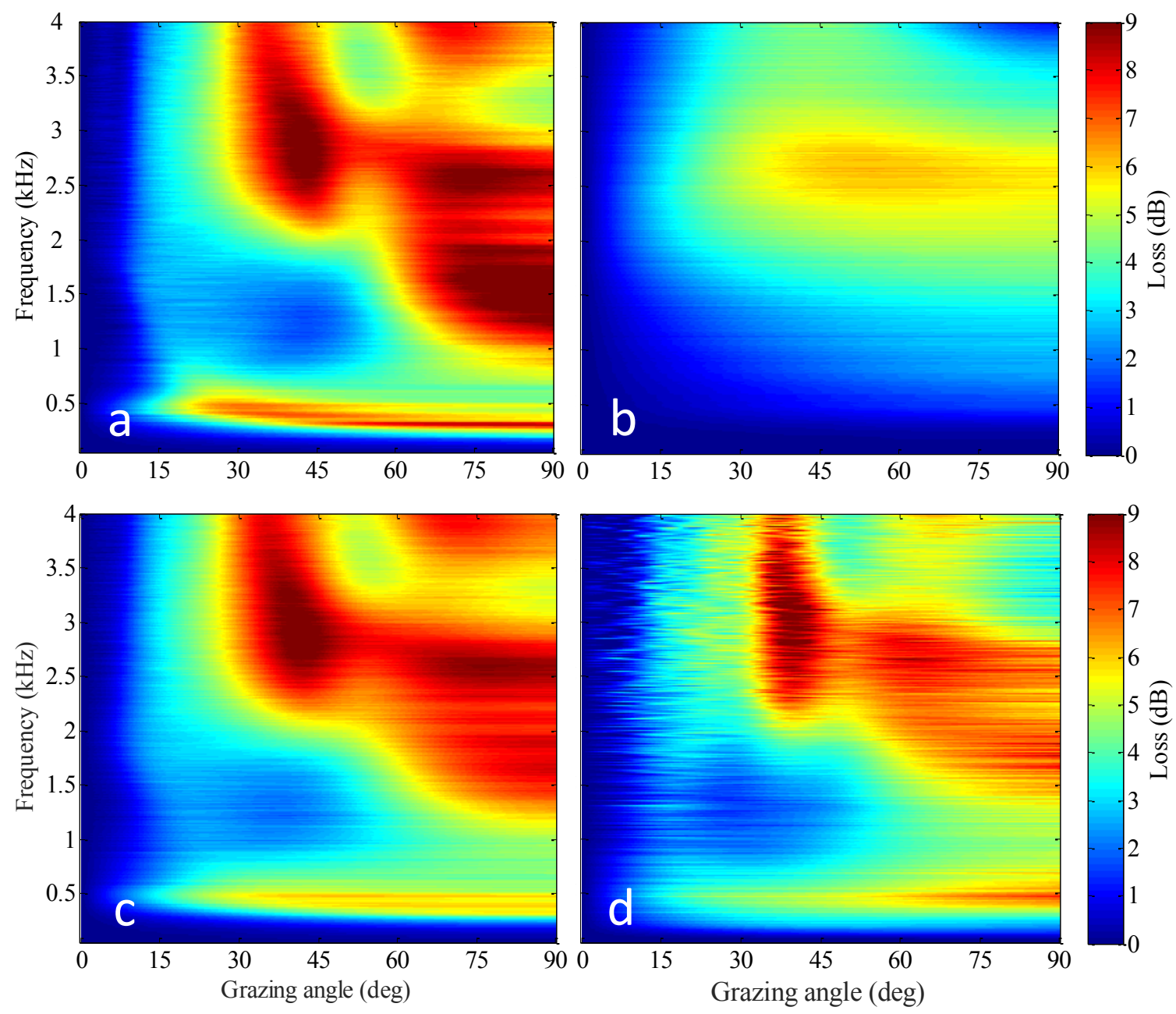

Figure 35. SLIVA data: BL estimated by HR-BL over 32 elements (a), by CBF over 8 (b) and 32 elements (c), and by HR-BL after extending the coherence function estimated from 8 sensors to 32 sensors by FBE (d). Despite the fact that the length of the array is being increased by a factor of 4 , the high sampling frequency affords the recovery of BL up to the array design frequency. 


\subsection{Summary}

The analysis of a model proposed by Harrison shows that the imaginary part of the spatial coherence function, measured by a vertical line array in a surface-ambientnoise field, is weakly dependent on the bottom reflection properties, and therefore on the signal frequency alone. The real part of the function contains the dependence on the bottom reflection properties, and therefore shows a greater dependence on frequency alone. Both the real and the imaginary part of the function depend strongly on the ratio of the sensor-pair spacing to signal wavelength $z / \lambda$. Furthermore, empirical analysis shows that the normalized coherence-function curves appear to vary smoothly with frequency.

Based on these considerations, in the case of a frequency-independent bottom reflection coefficient (such as that of a halfspace bottom), a simple technique can be envisioned to extend the coherence function to values of the sensor spacing that are beyond the physical length of the array, by making use of data at higher frequencies, and provided that the spacing to wavelength ratio is preserved. While some amount of error is expected when the technique is applied to a layered bottom, results show that, for the particular task of passive bottom-loss estimation, these errors are well within the margins of Harrison and Simons' technique. Processing of both simulated and measured data by FBE coupled with HR-BL show that the information lost by a short array can be at least partially recovered, while making a more efficient use of the large bandwidth afforded by modern acquisition systems. An important 
prerequisite for the application of the algorithm is that the data be free of interference from sources other than wind and wave noise. 


\section{Contributions}

This dissertation makes the following contributions to the field of underwater acoustics:

- Experimental verification of the performance and limitations of sub-meter arrays in bottom-loss estimation.

- Participation to the GLASS'13 and REP14-MED sea trials - Collaboration to the phases of: mission planning, equipment deployment and retrieval, data collection; processing of acquired ambient-noise data;

- numerical simulations and modeling;

o study of the sensitivity of the estimated bottom loss to the seabed parameters by geoacoustic inversion of the GLASS data.

- A derivation showing how, under some reasonable conditions, the bottom reflection coefficient can be obtained from the array spatial coherence function removing a shortcoming that is intrinsic to the beamforming used in Harrison and Simons' original technique. This is formulated starting from a known model of the marine ambient-noise spatial coherence function.

- A technique for increasing the angular resolution of the estimated reflection coefficient (and therefore of the derived bottom loss). This emerges as a natural consequence of the derivation, and is demonstrated on both synthetic and experimental data. 
- A technique for extending the coherence function to values of the sensor spacing that are beyond the physical length of the array, increasing the angular resolution of the estimated bottom loss.

This dissertation advances the capabilities of passive bottom-survey technology by arrays of length that is short compared to the signal wavelength, therefore providing essential groundwork, both theoretical and experimental, needed for migrating Harrison and Simons' technique from long arrays deployed by ships to arrays mounted on small AUVs. 


\section{Publications/proceedings related to this research}

This section reports a list of authored or co-authored publications or conference presentations that stemmed from this research work.

JOURNAL

- L. Muzi, J. Gebbie and M. Siderius, "Passive bottom reflection-loss estimation: A formal comparison between conventional beamforming and the high-resolution algorithm," in preparation for J. Acoust. Soc. Am. (2015).

- P. L. Nielsen, M. Siderius and L. Muzi, "Performance assessment of a short hydrophone array for seabed characterization using natural-made ambient noise," in preparation for IEEE Journal of Oceanic Engineering (2015).

- L. Muzi, M. Siderius, and P. L. Nielsen, "Frequency based noise coherence-function extension, and application to passive bottom-loss estimation," submitted to J. Acoust. Soc. Am. (2015).

- L. Muzi, M. Siderius, J. E. Quijano, and S. E. Dosso, "High-resolution bottom-loss estimation using the ambient-noise vertical coherence function", J. Acoust. Soc. Am. 137, 481-491 (2015).

- J. E. Quijano, S. E. Dosso, M. Siderius, and L. Muzi, "Coherence extrapolation for underwater ambient noise," J. Acoust. Soc. Am. 135, EL318-EL323 (2014).

- M. Siderius, L. Muzi, C. H. Harrison, and P. L. Nielsen, "Synthetic array processing of ocean ambient noise for higher resolution seabed bottom loss estimation," J. Acoust. Soc. Am. 133, EL149-EL155, (2013). 


\section{CONFERENCE}

- P. L. Nielsen, M. Siderius, and L. Muzi, "Glider-based seabed characterization using natural-made ambient noise," IEEE OCEANS 2015, Genova (Italy), May 18-21 2015.

- P. L. Nielsen, M. Siderius, and L. Muzi, "Performance assessment of a short hydrophone array for seabed characterization using natural-made ambient noise," J. Acoust. Soc. Am. 136, 2155 (2014).

- L. Muzi, M. Siderius, and P. L. Nielsen, "Experimental studies on passive bottom loss estimation from a compact array mounted on an autonomous underwater vehicle," 2nd International conference on underwater acoustics 2014, Rhodes (Greece), June 22-27 2014.

- L. Muzi and M. Siderius, "Synthetic-array beamforming for bottom-loss estimation using marine ambient noise," Proceedings of Meetings on Acoustics 19, p. 070033 (2013).

- L. Muzi and M. Siderius, "Adaptive beamforming techniques for bottom-loss estimation using marine ambient noise." J. Acoust. Soc. Am. 129, 2458 (2011).

- J. Gebbie, M. Siderius, L. Muzi, J. Paddock, "Extracting the Rayleigh reflection coefficient from the passive fathometer," Proceedings of MTS/IEEE OCEANS 2010, Seattle, September 20-23 2010.

- Muzi L, Siderius M, Gebbie J, Paddock J. "On the use of adaptive beam-forming techniques for geoacoustic inversion of marine ambient noise," Proceedings of MTS/IEEE OCEANS 2010 Seattle, September 20-23 2010. 


\section{REPORT}

- P. L. Nielsen, L. Muzi, M. Siderius, and J. H. Miller “Glider Acoustics Sensing of Sediments (GLASS): Experiments and data analysis", CMRE report, CMRE-FR2013-025. 


\section{References}

1. R. J. Urick, Principles of Underwater Sound, 3rd Edition, Peninsula Pub., Aug. 1996.

2. F. B. Jensen, W. A. Kuperman, M. B. Porter, and H. Schmidt, "Bottom loss," Section 1.6 in Computational Ocean Acoustics (Modern Acoustics and Signal Processing), 2nd ed., 38-50, Springer (2011).

3. R. Hamson, "The modelling of ambient noise due to shipping and wind sources in complex environments," Applied Acoustics 51, 251-287 (1997).

4. E. L. Hamilton, "Geoacoustic modeling of the sea floor," J. Acoust. Soc. Am. 68, 1313-1340 (1980).

5. E. L. Hamilton and R. T. Bachman, "Sound velocity and related properties of marine sediments," J. Acoust. Soc. Am. 72, 1891-1904 (1982).

6. C. Ferla and F. B. Jensen, "Are current environmental databases adequate for sonar predictions in shallow water?" Impact of Littoral Environmental Variability on Acoustic Predictions and Sonar Performance, Eds. N.G. Pace and F. B. Jensen, Kluwer Academic Publishers, 555-562 (2002).

7. M. D. Collins, W. A. Kuperman, and H. Schmidt, "Nonlinear inversion for oceanbottom properties," J. Acoust. Soc. Am. 92, 2770-2783 (1992).

8. S. E. Dosso, M. L. Yeremy, J. M. Ozard, and N. R. Chapman, "Estimation of oceanbottom properties by matched-field inversion of acoustic field data," IEEE J. Ocean. Eng. 18, 232-239 (1993).

9. P. Gerstoft, "Inversion of seismoacoustic data using genetic algorithms and a posteriori probability distributions," J. Acoust. Soc. Am.0001-4966 95, 770-781 (1994). 
10. A. Caiti, S. M. Jesus, and Å. Kristensen, "Geoacoustic seafloor exploration with a towed array in a shallow water area of the Strait of Sicily," IEEE J. Ocean. Eng.0364-9059 21, 355-366 (1996).

11. M. Siderius, P. L. Nielsen, and P. Gerstoft, "Range-dependent seabed characterization by inversion of acoustic data from a towed receiver array," J. Acoust. Soc. Am. 112, 1523-1535 (2002).

12. M. R. Fallat, P. L. Nielsen, S. E. Dosso, and M. Siderius, "Geoacoustic characterization of a range-dependent ocean environment using towed array data," IEEE J. Ocean. Eng. 30, 198-206 (2005).

13. D. P. Knobles, R. A. Koch, L. A. Thompson, K. C. Focke, and P. E. Eisman, "Broadband sound propagation in shallow water and geoacoustic inversion," J. Acoust. Soc. Am. 113, 205-222 (2003).

14. R. A. Koch and D. P. Knobles, "Geoacoustic inversion with ships as sources," J. Acoust. Soc. Am. 117, 626-637 (2005).

15. D. Tollefsen, M. J. Wilmut, and N. R. Chapman, "Estimates of geoacoustic model parameters from inversions of horizontal and vertical line array data," IEEE J. Ocean. Eng. 30, 764-772 (2005).

16. R. M. S. Barlee, N. R. Chapman, and M. J. Wilmut, "Geoacoustic model parameter estimation using a bottom moored hydrophone array," IEEE J. Ocean. Eng.0364$905930,773-783$ (2005).

17. S. E. Dosso, P. L. Nielsen, and M. J. Wilmut, "Data error covariance in matched-field geoacoustic inversion," J. Acoust. Soc. Am. 119, 208-219 (2006).

18. U.S. Supreme Court, "Winter, secretary of the Navy, et al. v Natural Resources Defense Council, Inc., et al.," in Supreme Court case, (Washington, DC), Oct. 2008. 
19. C. H. Harrison and D. G. Simons, "Geoacoustic inversion of ambient noise: A simple method," J. Acoust. Soc. Am., 112, 1377-1389 (2002).

20. M. Siderius, C. H. Harrison, M. B. Porter, "A passive fathometer technique for imaging seabed layering using ambient noise," J. Acoust. Soc. Am. 120, 1315-1323 (2006).

21. J. E. Quijano, S. E. Dosso, J. Dettmer, L. M. Zurk, M. Siderius, and C. H. Harrison, "Bayesian geoacoustic inversion using wind-driven ambient noise," J. Acoust. Soc. Am. 131, 2658-2667 (2012).

22. D. H. Johnson and D. E. Dudgeon, Array signal processing concepts and techniques, 65-89, Prentice-Hall, (1993).

23. H. Cox, R. Zeskind, and M. Owen, "Robust adaptive beamforming." IEEE Transactions on Acoustics, Speech, and Signal Processing [see also IEEE Transactions on Signal Processing], 35, 1365-1376 (1987).

24. M. Siderius and C. Harrison, "High-Frequency geoacoustic inversion of ambient noise data using short arrays," in High Frequency Ocean Acoustics Conference, M. B. Porter, M. Siderius, and W. A. Kuperman, Eds., AIP 728, 22-31, (2004).

25. C.H. Harrison and A. Baldacci, "Bottom reflection properties deduced from ambient noise: Simulation of a processing technique," SACLANTCEN Memorandum, SM-392, CMRE (2002).

26. V. Young, "Using a vertical line array and ambient noise o obtain measurements of seafloor reflection loss," Report SR-410, CMRE (2005).

27. J. Arvelo, "Aggressive adaptive beamforming for ambient noise inversion with a limited-aperture sonar on an autonomous platform," POMA 5, 055003 (2008). 
28. L. Muzi, M. Siderius, J. Gebbie, and J. Paddock, "On the use of adaptive beamforming techniques for geoacoustic inversion of marine ambient noise," Proceedings of OCEANS 2010, MTS/IEEE Seattle, September 20-23 2010.

29. L. Muzi and M. Siderius, "Adaptive beamforming techniques for bottom-loss estimation using marine ambient noise." J. Acoust. Soc. Am. 129, 2458 (2011).

30. P. L. Nielsen, L. Muzi, M. Siderius, and J. H. Miller "Glider Acoustics Sensing of Sediments (GLASS): Experiments and data analysis", CMRE report, CMRE-FR2013-025.

31. P. L. Nielsen, M. Siderius, and L. Muzi, "Glider-based seabed characterization using natural-made ambient noise," IEEE OCEANS 2015, Genova (Italy), May 18-21 2015.

32. M. Siderius, L. Muzi, C. H. Harrison, and P. Nielsen, "Synthetic array processing of ocean abmbient noise for higher resolution seabed bottom loss estimation," J. Acoust. Soc. Am. 133, EL149-EL155, (2013).

33. L. Muzi and M. Siderius, "Synthetic-array beamforming for bottom-loss estimation using marine ambient noise," Proceedings of Meetings on Acoustics 19, p. 070033 (2013).

34. L. Muzi, M. Siderius, J. E. Quijano, and S. E. Dosso, "High-resolution bottom-loss estimation using the ambient-noise vertical coherence function", J. Acoust. Soc. Am. 137, 481-491 (2015).

35. J. E. Quijano, S. E. Dosso, M. Siderius, and L. Muzi, "Coherence extrapolation for underwater ambient noise," J. Acoust. Soc. Am. 135, EL318-EL323 (2014).

36. L. Muzi, M. Siderius, and P. L. Nielsen, "Experimental studies on passive bottom loss estimation from a compact array mounted on an autonomous underwater 
vehicle," 2nd International conference on underwater acoustics 2014, Rhodes (Greece), June 22-27 2014.

37. L. Muzi, M. Siderius and P. L. Nielsen, "Frequency based noise coherence-function extension, and application to passive bottom-loss estimation", submitted to J. Acoust. Soc. Am. (2015).

38. H. L. Van Trees, Optimum Array Processing (Detection, Estimation, and Modulation Theory, Part IV), 1st ed., Wiley-Interscience, March 2002.

39. 22. D. H. Johnson and D. E. Dudgeon, Array signal processing concepts and techniques, Prentice-Hall, (1993).

40. D. G. Manolakis, V. K. Ingle, and S. M. Kogon, "Array processing," Ch.11 in Statistical and adaptive signal processing: Spectral Estimation, Signal Modeling, Adaptive Filtering and Array Processing, 1st ed., Artech House Inc., April 2005.

41. D. G. Manolakis, V. K. Ingle, and S. M. Kogon, "Least squares filtering and prediction," Ch.8 in Statistical and adaptive signal processing: Spectral Estimation, Signal Modeling, Adaptive Filtering and Array Processing, 1st ed., Artech House Inc., April 2005.

42. D. G. Manolakis, V. K. Ingle, and S. M. Kogon, "Adaptive filters," Ch.10 in Statistical and adaptive signal processing: Spectral Estimation, Signal Modeling, Adaptive Filtering and Array Processing, 1st ed., Artech House Inc., April 2005.

43. H. Krim and M. Viberg, "Two decades of array signal processing research: the parametric approach," IEEE Signal Processing Magazine, vol. 13, no. 4, pp. 67-94, July 1996.

44. O. L. Frost, "An algorithm for linearly constrained adaptive array processing," Proceedings of the IEEE, vol. 60, no. 8, pp. 926-935, 1972. 
45. H. L. Van Trees, "LMS Algorithms", Section 7.7 in Optimum Array Processing (Detection, Estimation, and Modulation Theory, Part IV), 1st ed., WileyInterscience, March 2002.

46. D. G. Simons, C. Moll, and C. H. Harrison, "Inversion of shallow water ambient noise data by means of differential evolution as a global search method," in Acoustic Sensing Techniques for the Shallow Water Environment, 265-279, Springer Netherlands (2006).

47. C. H. Harrison, "Sub-bottom profiling using ocean ambient noise," J. Acoust. Soc. Am. 115, 1505-1515 (2004).

48. C. H. Harrison, "Performance and limitations of spectral factorization for ambient noise sub-bottom profiling," J. Acoust. Soc. Am. 118, 2913-2923 (2005).

49. C. H. Harrison and M. Siderius, "Bottom profiling by correlating beam-steered noise sequences," J. Acoust. Soc. Am. 123, 1282-1296 (2008).

50. J. I. Arvelo, "Robustness and constraints of ambient noise inversion," J. Acoust. Soc. Am. 123, 679-686 (2008).

51. J. Quijano, S. Dosso, J. Dettmer, L. Zurk and M. Siderius, "Bayesian Ambient Noise Inversion for Geoacoustic Uncertainty Estimation - Final report", ONR report, available at http://www.onr.navy.mil/reports/FY12/oaquijan.pdf (2013).

52. P. Gerstoft, W. S. Hodgkiss, M. Siderius, C.-F. Huang, and C. H. Harrison, "Passive fathometer processing," J. Acoust. Soc. Am. 123, 1297-1305 (2008).

53. M. Siderius, H. Song, P. Gerstoft, W. S. Hodgkiss, P. Hursky, and C. Harrison, “Adaptive passive fathometer processing," J. Acoust. Soc. Am. 127, 2193-2200 (2010). 
54. S. L. Means and M. Siderius, "Effects of sea-surface conditions on passive fathometry and bottom characterization," J. Acoust. Soc. Am. 126, 2234-2241 (2009).

55. J. Gebbie, M. Siderius, L. Muzi, J. Paddock, "Extracting the Rayleigh reflection coefficient from the passive fathometer," Proceedings of OCEANS 2010, MTS/IEEE Seattle, September 20-23 2010.

56. J. Traer, P. Gerstoft, and W. S. Hodgkiss, "Ocean bottom profiling with ambient noise: A model for the passive fathometer," J. Acoust. Soc. Am. 129, 1825-1836 (2011).

57. P. E. Hagen, N. Storkersen, K. Vestgard, and P. Kartvedt, "The HUGIN 1000 autonomous underwater vehicle for military applications," in OCEANS 20032 , 1141-1145, IEEE, Sept. 2003.

58. J. P. Wilkinson and P. Wadhams, "Modeling the flow of oil under sea ice: a role for the AUV," in Autonomous Underwater Vehicles, 2008. AUV 2008.IEEE/OES, 1-5, IEEE, Oct. 2008.

59. P. G. Fernandes, P. Stevenson, A. S. Brierley, F. Armstrong, and Simmonds, "Autonomous underwater vehicles: future platforms for fisheries acoustics," ICES Journal of Marine Science: Journal du Conseil, 60, 684-691 (2003).

60. H. Iwakami, T. Ura, K. Asakawa, T. Fujii, Y. Nose, J. Kojima, Y. Shirasaki, T. Asai, S. Uchida, N. Higashi, and T. Fukuchi, "Approaching whales by autonomous underwater vehicle," Marine Technology Society Journal, 36, 80-85 (2002).

61. A. Caffaz, A. Turetta, J.R. Potter, G. Casalino, A. Munafo, H. Tay, P. Guerrini, A.Caiti, V. Calabro, and A. Maguer, "The enhanced Folaga: a hybrid AUV with modular payloads," in Roberts and Sutton, Eds., Further Advances in Unmanned Marine Vehicles, 309-330, Institution of Engineering and Technology, Jan. 2012. 
62. M. Siderius, "Using practical supergain for passive imaging with noise," J. Acoust. Soc. Am. 131, EL14-EL20 (2012).

63. H. Cox, R. Zeskind, and T. Kooij, "Practical supergain," IEEE Transactions on Acoustics, Speech and Signal Processing 34, 393-398 (1986).

64. B. F. Cron and C. H. Sherman, "Spatial-Correlation functions for various noise models," J. Acoust. Soc. Am. 34, 1732-1736, 1962.

65. B. F. Cron, "Addendum: Spatial-Correlation functions for various noise models," J. Acoust. Soc. Am. 38, 885 (1965).

66. W. S. Liggett and M. J. Jacobson, "Noise covariance and vertical directivity in a deep ocean," J. Acoust. Soc. Am. 39, 280-288 (1966).

67. H. Cox, "Spatial correlation in arbitrary noise fields with application to ambient sea noise," J. Acoust. Soc. Am. 54, 1289-1301 (1973).

68. W. A. Kuperman and F. Ingenito, "Spatial correlation of surface generated noise in a stratified ocean," J. Acoust. Soc. Am. 67, 1988-1996, 1980.

69. M. J. Buckingham, "A theoretical model of ambient noise in a low-loss, shallow water channel," J. Acoust. Soc. Am. 67, 1186-1192 (1980).

70. M. J. Buckingham and S. A. S. Jones, "A new shallow-ocean technique for determining the critical angle of the seabed from the vertical directionality of the ambient noise in the water column," J. Acoust. Soc. Am. 81, 938-946 (1986).

71. H. Nakahara, "A systematic study of theoretical relations between spatial correlation and green's function in one-, two- and three-dimensional random scalar wavefields," Geophysical Journal International, 167, 1097-1105 (2006). 
72. S. C. Walker and M. J. Buckingham, "Spatial coherence and cross correlation of three-dimensional ambient noise fields in the ocean," J. Acoust. Soc. Am. 131, 1079-1086 (2012).

73. C. H. Harrison, "Formulas for ambient noise level and coherence," J. Acoust. Soc. Am. 99, 2055-2066 (1996).

74. C.H. Harrison, "Noise directionality for surface sources in range-dependent environments," J. Acoust. Soc. Am. 102, 2655-2662 (1997).

75. D. R. Barclay and M. J. Buckingham, "Depth dependence of wind-driven, broadband ambient noise in the Philippine Sea," J. Acoust. Soc. Am. 133, 62-71 (2013).

76. G. B. Deane, M. J. Buckingham and T. Tindle, Vertical coherence of ambient noise in shallow water overlying a fluid seabed", J. Acoust. Soc. Am. 102, 3413-3424 (1997).

77. H. Schmidt, OASES Version 3.1 User Guide and Reference Manual, Massachusetts Institute of Technology, Cambridge, MA, (2004).

78. P. Nielsen, J.H. Miller, M. Siderius, S. Crocker and J. Giard, “GLASS'12 - The Glider Acoustics Sensing of Sediments Experiment 2012," Technical Report, CMRE-FR-212-07, CMRE (2012).

79. Papoulis, "A new algorithm in spectral analysis and band-limited extrapolation," IEEE Transactions on Circuits and Systems, 22, no. 9, 735-742 (1975).

80. Jain and S. Ranganath, "Extrapolation algorithms for discrete signals with application in spectral estimation," IEEE Trans. Acoust. Speech Signal Process. 29, 830-845 (1981). 
81. J. A. Cadzow, "An extrapolation procedure for bandlimited signals," IEEE Trans. Acoust. Speech Signal Process. 27, 4-12 (1979).

82. D. G. Manolakis, V. K. Ingle, and S. M. Kogon, "Gradients and optimization in complex space," Appendix B in Statistical and adaptive signal processing: Spectral Estimation, Signal Modeling, Adaptive Filtering and Array Processing, 1st ed., Artech House Inc., April 2005.

83. F. B. Jensen, W. A. Kuperman, M. B. Porter, and H. Schmidt, "Normal modes," in Computational Ocean Acoustics (Modern Acoustics and Signal Processing), 2nd ed. (Springer, New York, 2011), Chap.5, p.340. 